\title{
A GENERAL ALGEBRAIC APPROACH TO STEENROD OPERATIONS
}

\author{
by \\ J. Peter May
}

1. Introduction. Since the introduction of the Steenrod operations in the cohomology of topological spaces, it has become clear that similar operations exist in a variety of other situations. For example, there are Steenrod operations in the cohomology of simplicial restricted Lie algebras, in the cohomology of cocommutative Hopf algebras, and in the homology of infinite loop spaces (where they were introduced $\bmod 2$ by Araki and Kudo [3] and $\bmod p, p>2$, by Dyer and Lashof [6]).

The purpose of this expository paper is to develop a general algebraic setting in which all such operations can be studied simultaneously. This approach allows a single proof, applicable to all of the above examples, of the basic properties of the operations, including the Adem relations. In contrast to categorical treatments of Steenrod operations, the elegant proofs developed by Steenrod [25-30] actually simplify somewhat in our algebraic setting. Further, even the most general existing categorical study of Steenrod operations, that of Epstein [7], cannot be applied to iterated loop spaces.

We emphasize that this is an expository paper. Although a number of new results and new proofs of old results are scattered throughout, the only real claim to originality lies in the basic context. We have chosen to give complete proofs of all results since a large number of minor simplifications in the arguments allows a substantial simplification of the theory as a whole. We have also included a number of topological results which should be well-known but appear not to be in the litera- 
ture. In particular, in section 10 , we give a quick complete calculation of the $\bmod p$ cohomology Bockstein spectral sequence of $K(\pi, n)^{\prime}$ s and show that Serre's simple proof [23] of the axiomatization of the mod 2 Steen rod operations applies with only slight modifications to the case $p>2$.

The general theory is presented in the first five sections. Most of the proofs in sections 1,2, and 4 are based on those of Steenrod [25-30], and those of section 3 are simplifications of arguments of Dyer and Lashof [6 ]. Via acyclic models and a lemma due to Dold [5], the theory is applied to several simplicial categories and to topological spaces in sections 7 and 8 . The standard properties of the Steenrod operations in spaces, except $\mathrm{P}^{0}=1$, drop out of the algebraic theory, and $P^{\circ}=1$ is shown to follow from these properties. In contrast, we prove that $\mathrm{P}^{\circ}=0$ on the cohomology of simplicial restricted Lie algebras. The theory is applied to the cohomology of cocommutative Hopf algebras in section 11; the operations here are important in the study of the cohomology of the Steenrod algebra $[13,18]$. The present analysis arose out of work on iterated loop spaces, but this application will appear elsewhere. The material of sections 6 and 9 , which is peripheral to the study of Steenrod operations, is presented here with a view towards this application. 


\section{Algebraic preliminaries; equivariant homology}

Let $\Lambda$ be a commutative (ungraded) ring. By a $\Lambda$-complex, we understand a $Z$-graded differential $\Lambda$-module, graded by subscripts, with differential of degree minus one. We say that a $\Lambda$-complex $K$ is positive if $K_{q}=0$ for $q<0$ and negative if $\mathrm{K}_{\mathrm{q}}=0$ for $\mathrm{q}>0$. We use $\mathrm{Z}$-graded complexes in order that our theory can be applied equally well to homology and to cohomology. The exposition will be geared to homology, where the notation is slightly simpler, and the notations appropriate for cohomology will be given in section 5 . We give some elementary homological lemmas in this section; these extract the slight amount of information about the homology of groups that is needed for the development of the Steenrod operations.

If $\pi$ is a group, we let $\Lambda \pi$ denote its group ring over $\Lambda$. We shall generally speak of $\Lambda \pi$-morphisms rather than $\pi$-equivariant $\Lambda$-morphisms, and we shall speak of $\pi$-morphisms when $\Lambda$ is understood. Let $\Sigma_{r}$ denote the symmetric group on $r$ letters, and let $\pi \subset \Sigma_{r^{*}}$. For $q \in Z$, let $\Lambda(q)$ denote the $\Lambda \pi$-module which is $\Lambda$ as a $\Lambda$-module and has the $\Lambda \pi$-action determined by $\sigma \lambda=(-1)^{\mathrm{qs}(\sigma)} \lambda$, where $(-1)^{\mathrm{s}(\sigma)}$ is the sign of $\sigma \in \pi$. If $M$ is a $\Lambda \pi$-module, let $M(q)$ denote the $\Lambda \pi-$ module $M \otimes \Lambda(q)$ with the diagonal action $\sigma(m \otimes \lambda)=\sigma m \otimes \sigma \lambda$ (where $\otimes=\bigotimes_{\Lambda}$ ). If $K$ is a $\Lambda$-complex, let $K^{r}=K \otimes \ldots \otimes K, r$ factors $K$. Via permutation of factors, with the standard sign convention, $K^{\mathbf{r}}$ becomes a $\Lambda \pi-c o m p l e x$ for $\pi \subset \Sigma_{r}$, and $K^{r}(q)$ is defined.

Let I denote the $\Lambda$-free $\Lambda$-complex which has two basis elements $e_{o}$ and $e_{1}$ of degree zero, one basis element e of degree one, and differential $d(e)=e_{1}-e_{0}$. If $\Gamma$ is a Hopf algebra over $\Lambda$ and $I$ is given the trivial $\Gamma$-module structure, $y a=\varepsilon(\gamma) a$ for $\gamma \in \Gamma$ and $a \in I$, then the notion of a $\Gamma$-homotopy $h: f \simeq g$, where $f, g: K \rightarrow L$ are morphisms of $\Gamma$-complexes, is equivalent to the notion of a $\Gamma$-morphism $H: I \otimes K \rightarrow L$ such that $H\left(e_{1} \otimes k\right)=f(k)$ and $H\left(e_{0} \otimes k\right)=g(k)$, where 
$I \otimes K$ is given the diagonal $\Gamma$-action. In fact, $H$ determines $h$ by $h(k)=H(e \otimes k)$ and conversely.

With these notations, we have the following lemma. In all parts, $\Lambda \pi$ acts diagonally on tensor products.

Lemma 1.1. Let $\pi \subset \Sigma_{\mathrm{r}}$ and let $\mathrm{V}$ be a positive $\Lambda \pi$-free complex.

(i) There exists a $\Lambda \pi$-morphism $h: I \otimes V \rightarrow V \otimes I^{r}$ such that $h\left(e_{0} \otimes v\right)=v \otimes e_{0}^{r}$ and $h\left(e_{1} \otimes v\right)=v \otimes e_{1}^{r}$ for all $v \in V$.

(ii) If $\mathrm{f}, \mathrm{g}: \mathrm{K} \rightarrow \mathrm{L}$ are $\Lambda$-homotopic morphisms of $\Lambda$-complexes, then $1 \otimes f^{r}, 1 \otimes g^{r}: V \otimes K^{r} \rightarrow V \otimes L^{r}$ are $\Lambda \pi$-homotopic morphisms of $\Lambda \pi-$ complexes.

(iii) If $\Lambda$ is a field and $K$ is a $\Lambda$-complex, then $K$ is $\Lambda$-homotopy equivalent to $H(K)$ and $V \otimes K^{r}$ is $\Lambda \pi$-homotopy equivalent to $V \otimes H(K)^{r}$.

(iv) Let $v \in V$ satisfy $d(v \otimes 1)=0$ in $V \otimes_{\pi} \Lambda$; let $K$ be a $\Lambda$-complex and let $\mathrm{a}, \mathrm{b} \in \mathrm{K}_{\mathrm{q}}$ be homologous cycles. Then $\mathrm{v} \otimes \mathrm{a}^{\mathrm{r}}$ and $\mathrm{v} \otimes \mathrm{b}^{\mathrm{r}}$ are homologous cycles of $V \otimes_{\pi} K^{r}(q)$.

Proof. (i) Let $\varepsilon: I \rightarrow \Lambda$ be the augmentation $\varepsilon\left(e_{0}\right)=1=\varepsilon\left(e_{1}\right)$, and let $J=\operatorname{Ker}\left(\varepsilon^{r}\right), \quad \varepsilon^{r}: I^{r} \rightarrow \Lambda^{r}=\Lambda$. Define $k: V \rightarrow V \otimes J$ by $k(v)=v \otimes\left(e_{1}^{r}-e_{0}^{r}\right)$. Since $H(J)=0, H(V \otimes J)=0$. Define a $\Lambda \pi$-homotopy $s: V \rightarrow V \otimes J$ from $k$ to the zero map by induction on degree as follows. Let $s_{-1}=0$; given $s_{i-1}: V_{i-1} \rightarrow(V \otimes J)_{i}$, we find easily that $d_{i}\left(k_{i}-s_{i-1} d_{i}\right)=0$. Let $\left\{x_{j}\right\}$ be a $\Lambda \pi$-basis for $V_{i} ;$ for $x \in\left\{x_{j}\right\}$, choose $s_{i}(x)$ such that $d_{i+1} s_{i}(x)=k_{i}(x)-s_{i-1} d_{i}(x)$, and extend $s_{i}$ to all of $V_{i}$ by $\pi$-equivariance. The desired $\Lambda \pi$-morphism $h$ is obtained by letting $h(e \otimes v)=s(v)$ for $v \in V$.

(ii) Let $t: I \otimes K \rightarrow L$ determine a $\Lambda$-homotopy from $f$ to $g$. Then the following composite is a $\Lambda \pi$-morphism which determines a $\Lambda \pi$-homotopy from $1 \otimes \mathrm{f}^{\mathbf{r}}$ to $1 \otimes g^{r}:$

$$
I \otimes V \otimes K^{r} \stackrel{h \otimes 1}{\longrightarrow} V \otimes I^{r} \otimes K^{r} \stackrel{1 \otimes u}{\longrightarrow} V \otimes(I \otimes K)^{r} \stackrel{1 \otimes t^{r}}{\longrightarrow} V \otimes L^{r} .
$$

where $u: I^{r} \otimes K^{r} \rightarrow(I \otimes K)^{r}$ is the evident shuffling isomorphism. 
(iii) Define $\mathrm{f}: \mathrm{H}(\mathrm{K}) \rightarrow \mathrm{K}$ by sending each element of a basis for $\mathrm{H}(\mathrm{K})$ to a chosen representative cycle. $K \cong \operatorname{Im} f \oplus$ Coker $f$ as a $\Lambda$-complex and Coker $f$ is acyclic and the refore contractible since $\Lambda$ is a field. The first half follows and implies the second half by (ii).

(iv) Define a morphism of $\Lambda$-complexes $f: I \rightarrow K$, of degree $q$, by $f\left(e_{1}\right)=a$, $f\left(e_{0}\right)=b$, and $f(e)=(-1)^{q} c$, where $d(c)=a-b$ in $K$ (the sign ensures that $\left.\mathrm{d} f(e)=(-1)^{q} f d(e)\right)$. Let $F: I \otimes V \rightarrow V \otimes K^{r}(q)$ be the composite $I \otimes V \stackrel{h}{\longrightarrow} V \otimes I^{r} \stackrel{1 \otimes f^{r}}{\longrightarrow} V \otimes K^{r}(q)$. A check of signs shows that $f^{r}$ is a $\Lambda \pi$-morphism, hence that $F$ is a morphism of $\Lambda \pi$-complexes of degree $q r$. By (i), we find that

$$
F\left(e_{i} \otimes v\right)=\left(1 \otimes f^{r}\right)\left(v \otimes e_{i}^{r}\right)=(-1)^{q r d e g} v_{v} \otimes f\left(e_{i}\right)^{r}, i=0 \text { or } 1 .
$$

Since $\pi$ operates trivially on $I$ and $d(v \otimes 1)=0$ in $V \otimes_{\pi} \Lambda$, we have that $\mathrm{d}(\mathrm{e} \otimes \mathrm{v})=\left(\mathrm{e}_{1}-\mathrm{e}_{\mathrm{o}}\right) \otimes_{\mathrm{v}}$ in $\mathrm{I} \otimes_{\pi} \mathrm{V}$. Thus, in $\mathrm{V} \otimes_{\pi} \mathrm{K}^{\mathrm{r}}(\mathrm{q})$,

$$
\mathrm{dF}(\mathrm{e} \otimes \mathrm{v})=(-1)^{\mathrm{qr}} F\left(\mathrm{e}_{1} \otimes \mathrm{v}-\mathrm{e}_{\mathrm{o}} \otimes \mathrm{v}\right)=(-1)^{\mathrm{qr}(\mathrm{deg} \mathrm{v}+1)}\left(\mathrm{v} \otimes \mathrm{a}^{\mathrm{r}}-\mathrm{v} \otimes \mathrm{b}^{\mathrm{r}}\right),
$$

and this proves the result.

We now consider the cyclic group $\pi$ of prime order $p$. We recall the definition of the standard $\Lambda \pi-$ free resolution $W=W(p, \Lambda)$ of $\Lambda$.

Definition 1.2. Let $\pi$ be the cyclic group of prime order $p$ with generator $\alpha$. Let $W_{i}$ be $\Lambda \pi$-free on one generator $e_{i}$, $i \geq 0$. Let $N=1+\alpha+\ldots+\alpha^{p-1}$ and $T=\alpha-1$ in $\Lambda \pi$. Define a differential $d$, augmentation $\varepsilon$, and coproduct $\psi$ on $W$ by the formulas

$$
\begin{aligned}
& d\left(e_{2 i+1}\right)=T e_{2 i} \text { and } d\left(e_{2 i}\right)=N e_{2 i-1} ; \quad \varepsilon\left(\alpha^{j} e_{o}\right)=1 ; \\
& \psi\left(e_{2 i+1}\right)=\sum_{j+k=i} e_{2 j} \otimes e_{2 k+1}+\sum_{j+k=i} e_{2 j+1} \otimes \alpha e_{2 k} \text { and } \\
& \psi\left(e_{2 i}\right)=\sum_{j+k=i} e_{2 j} \otimes e_{2 k}+\sum_{j+k=i-1} \quad \sum_{r<s<p} \alpha^{r} e_{2 j+1} \otimes \alpha^{s} e_{2 k+1} .
\end{aligned}
$$

Then $W$ is a differential $\Lambda \pi$-coalgebra and a $\Lambda \pi$-free resolution of $\Lambda$. When necessary for clarity, we shall write $W(p, \Lambda)$ for $W$. Of course, 
$W(p, \Lambda)=W(p, z) \otimes \Lambda$. The structure of $W\left(p, z_{p}\right)$ shows that $H_{* *}\left(\pi ; z_{p}\right)=H\left(W\left(p, z_{p}\right) \otimes_{\pi} Z_{p}\right)$ is given, with its Bockstein operation $\beta$ and coproduct $\psi$, by the formula

(2) $H_{*}\left(\pi ; Z_{p}\right)$ has $Z_{p}$-basis $\left\{e_{i} \mid i \geq 0\right\}$ such that $\beta\left(e_{2 i}\right)=e_{2 i-1}$ and $\psi\left(e_{i}\right)=\sum_{j+k=i} e_{j} \otimes e_{k}$ if $p=2$ or $i$ is odd, $\psi\left(e_{2 i}\right)=\sum_{j+k=i} e_{2 j} \otimes e_{2 k}$ if $p>2$.

We embed $\pi$ in $\Sigma_{p}$ by $\alpha(1, \ldots, p)=(p, 1, \ldots, p-1)$, where $\Sigma_{p}$ acts on $\{1, \ldots, p\}$. We then have the following lemma.

Lemma 1.3. Let $w^{(n)}=\sum_{i \leq n} w_{i}$ be the n-skeleton of $W=W\left(p, z_{p}\right)$. Let $G$ be any set of left coset representatives for $\pi$ in $\Sigma_{p}$. Let $K$ be a $Z_{p}$-module with totally ordered basis $\left\{x_{j} \mid j \in J\right\}$. Let $A \subset K^{P}$ have basis $\left\{x_{j}^{P} \mid j \in J\right\}$ and let $B \subset K^{p}$ have basis $\left\{x_{\gamma\left(j_{p}\right)} \mid \gamma \in G, j_{1} \leq \ldots \leq j_{p}, j_{1}<j_{p}\right\}$. Then

$$
H\left(W^{(n)} \otimes K_{\pi}\right)=\left(\underset{i=0}{\oplus} e_{i} \otimes A\right) \oplus\left(e_{0} \otimes B\right) \oplus\left(K e r d_{n} \otimes B\right), \quad d_{n}: W_{n} \rightarrow W_{n-1} .
$$

Proof. It is easy to see that $K^{\mathrm{p}}$ is isomorphic as a $Z_{\mathrm{P}}{ }^{\pi \text {-module to }}$ $A \oplus\left(Z_{p} \pi \otimes B\right)$, where $\pi$ acts trivially on $A$ and acts on $Z_{p} \pi \otimes B$ by its left action on $Z_{p} \pi$. Since $H\left(W^{(n)} \otimes_{\pi} A\right)=H\left(W^{(n)} \otimes_{\pi} Z_{p}\right) \otimes A \quad$ and $H\left(W^{(n)} \otimes_{\pi} Z_{p} \pi B\right)=H\left(W^{(n)}\right) \otimes B$, the result follows.

Recall that if $\pi$ is any subgroup of $\Sigma$ and if $\gamma \in N(\pi)$, the normalizer of $\pi$ in $\Sigma$, then conjugation by $\gamma$ defines a homomorphism $\gamma_{* k}: H(\pi ; M) \rightarrow H_{* k}(\pi ; M)$ for any $\Lambda \Sigma$-module $M . \quad \gamma_{*}$ is the map induced on homology from $\gamma_{\sharp} \otimes \gamma: W \otimes_{\pi} M \rightarrow W \otimes_{\pi} M$ where $W$ is any $\Lambda \pi$-free resolution of $\Lambda$ and $\gamma_{\#}: W \rightarrow W$ is any morphism of $\Lambda$-complexes such that $\gamma_{\#}(\sigma w)=\gamma \sigma \gamma^{-1} \gamma_{\#}(w)$ for $\sigma \in \pi$ and $w \in W$. (It is easy to verify that $\gamma_{\#}$ exists and that $\gamma_{*}$ is independent of the choice of $W$ and of $\gamma_{\#}$ ) Clearly $\ddot{\gamma}_{* *}=1$ if $\gamma \in \pi$ since we may then define $\gamma_{\#}(w)=\gamma w$ so that

$$
\left(\gamma_{\#} \otimes_{\pi} \gamma\right)(w \otimes m)=\gamma w \otimes \gamma m=w \otimes m, \quad w \in W \text { and } m \in M
$$


If $\pi \subset p \subset \Sigma$ and $\gamma \in N(\pi) \cap N(\rho)$, then the following diagram commutes:

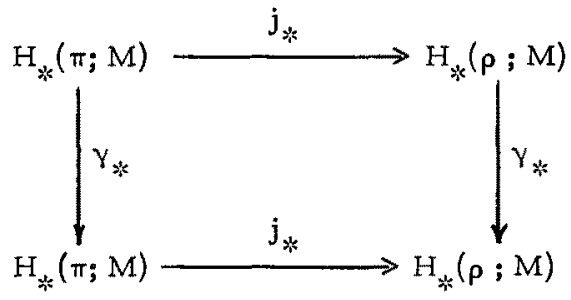

In fact, if $W$ is any $\Lambda \rho$-free resolution of $\Lambda$, then $W$ is also a $\Lambda \pi$-f ree resolution of $\Lambda$, and the above diagram results from the observation that $j_{*}$ is induced from $W \otimes_{\pi} M \rightarrow W \otimes_{\rho} M$. In particular, $j_{*}=j_{*} \gamma_{*}$ if $\gamma \in \rho$.

Lemma 1.4. Let $\pi$ be cyclic of prime order $p>2$ and let $q \in Z$. Consider $j_{*}: H_{*}\left(\pi ; z_{p}(q)\right) \longrightarrow H_{*}\left(\Sigma ; ; z_{p}(q)\right)$. Then

(i) If $q$ is even, $j_{*}\left(e_{i}\right)=0$ unless $i=2 t(p-1)$ or $i=2 t(p-1)-1$.

(ii) If $q$ is odd, $j_{* k}\left(e_{i}\right)=0$ unless $i=(2 t+1)(p-1)$ or $i=(2 t+1)(p-1)-1$.

Proof. Let $k$ gene rate the multiplicative subgroup of $z_{p}, k^{p-1}=1$. Let $\Sigma_{p}$ operate on $Z_{p}$ and define $\gamma \in \Sigma_{p}$ by $\gamma(i)=k i$. Then $\gamma a \gamma^{-1}=\alpha^{k}$ and $\gamma$ is an odd permutation in $N(\pi)$. Define $\gamma_{\#}: W \rightarrow W$ by

$$
\gamma_{\#}\left(e_{2 i}\right)=k^{i} e_{2 i} ; \quad \gamma_{\#}\left(e_{2 i+1}\right)=k^{i} \sum_{j=0}^{k-1} \alpha^{j} e_{2 i+1} ; \quad \gamma_{\#}\left(\sigma e_{i}\right)=\gamma \sigma \gamma^{-1} \gamma_{\#}\left(e_{i}\right), \quad \sigma \in \pi
$$

Then $\gamma_{\#} d=d \gamma_{\#}$ and $\gamma_{\#} \otimes \gamma$ induces the conjugation $\gamma_{*}$ on $H_{*}\left(\pi ; z_{p}(q)\right)$. Since $\gamma \in \Sigma_{p}, j_{*} \gamma_{*}=j_{*}$, hence $j_{*}\left(e_{i}-\gamma_{*} e_{i}\right)=0$ for all i. $\gamma$ operates by $(-1)^{q}$ on $Z_{p}(q)$ and therefore

$$
\gamma_{* k}\left(e_{2 i}\right)=(-1)^{q_{k} e^{i}} e_{2 i} \text { and } \gamma_{*}\left(e_{2 i+1}\right)=(-1)^{q_{k} i+1} e_{2 i+1} .
$$

Thus $j_{*}\left(e_{2 i}\right)=0$ unless $1-(-1)^{q_{k} i} \equiv 0 \bmod p$ and $j_{*}\left(e_{2 i+1}\right)=0$ unless $1-(-1)^{q} k^{i+1} \equiv 0 \bmod p$. Clearly $k^{i} \equiv 1 \bmod p$ if and only if $i=t(p-1)$ for some $t$ and $k^{i} \equiv-1 \bmod p$ if and only if $2 i=(2 t+1)(p-1)$ for some $t$. The result follows easily. 


\section{The definition and elementary properties of the operations}

We now define a large algebraic category $\zeta(p, n)$ on which the Steenrod operations will be defined. Steenrod operations will be obtained for particular categories of interest by obtaining functors to $\zeta(p, n)$. The interest of the integer $\mathrm{n}$ in the following definition lies solely in the applications to iterated loop spaces. For all other known applications, only the case $n=\infty$ is relevant.

Definitions 2.1. Let $\Lambda$ be a commutative ring, let $r$ be an integer, and let $\pi$ be a subgroup of $\Sigma_{r^{*}}$ Let $W$ be a $\Lambda \pi$-free resolution of $\Lambda$, let $V$ be a $\Lambda \Sigma_{r}$ free resolution of $\Lambda$, and let $\mathrm{j}: \mathrm{W} \rightarrow \mathrm{V}$ be a morphism of $\Lambda \pi$-complexes over $\Lambda$. Assume that $W_{0}=\Lambda \pi$ with generator $e_{0}$. Let $0 \leq n \leq \infty$ and let $W^{(n)}$ and $V^{(n)}$ denote the $n$-skeletons of $W$ and $V$. Define a category $\zeta(\pi, n, \Lambda)$ as follows. The objects of $\zeta(\pi, n, \Lambda)$ are pairs $(K, \theta)$, where $K$ is a homotopy associative differential $\Lambda$-algebra and $\theta: W^{(n)} \otimes K^{r} \rightarrow K$ is a morphism of $\Lambda \pi$-complexes such that

(i) The restriction of $\theta$ to $e_{0} \otimes K^{r}$ is $\Lambda$-homotopic to the iterated product $\mathrm{K}^{\mathrm{r}} \rightarrow \mathrm{K}$, associated in me fixed order, and

(ii) $\theta$ is $\Lambda \pi$-homotopic to a composite $W^{(n)} \otimes K^{r} \stackrel{j \otimes 1}{\longrightarrow} V^{(n)} \otimes K^{r} \stackrel{\emptyset}{\longrightarrow} K$, where $\emptyset$ is a morphism of $\Lambda \Sigma \Sigma_{\mathbf{r}}$-complexes.

A morphism $f:(K, \theta) \rightarrow\left(K^{\prime}, \theta^{\prime}\right)$ in $\zeta(\pi, n, \Lambda)$ is a morphism of $\Lambda$-complexes $\mathrm{f}, \mathrm{K} \rightarrow \mathrm{K}^{+}$such that the diagram

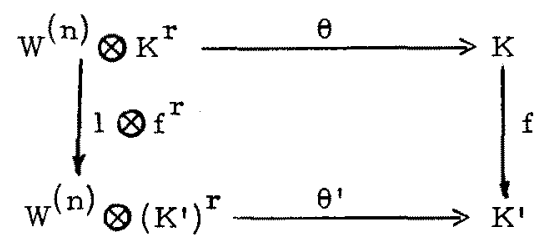

is $\Lambda \pi$-homotopy commutative. A morphism $f$ is said to be perfect if $\theta^{\prime}\left(1 \otimes f^{r}\right)=f \theta$, with no homotopy required, and $\mathcal{P}(\pi, n, \Lambda)$ denotes the subcategory of $\mathscr{b}(\pi, n, \Lambda)$ having the same objects $(K, \theta)$ and all perfect morphisms between them. $\Lambda$ is itself an object of $\zeta(\pi, n, \Lambda)$, with 
$\theta=\varepsilon \otimes 1: W^{(n)} \otimes \Lambda^{r} \longrightarrow \Lambda^{r}=\Lambda$, and an object $(K, \theta) \in \zeta(\pi, n, \Lambda)$ is said to be unital if $K$ has a two-sided homotopy identity e such that $\eta: \Lambda \rightarrow K, n(1)=e$, is a morphism in $\zeta(\pi, n, \Lambda)$. The tensor product of objects $(K, \theta)$ and $\left(L, \theta^{\prime}\right)$ in $\zeta(\pi, n, \Lambda)$ is the pair $(K \otimes L, \tilde{\theta})$, where $\widetilde{\theta}$ is the composite

$$
\begin{aligned}
W^{(n)} \otimes(K \otimes L)^{r} \stackrel{\psi \otimes U}{\longrightarrow} W^{(n)} \otimes W^{(n)} \otimes K^{r} \otimes L^{r} \stackrel{I \otimes T \otimes 1}{\longrightarrow} W^{(n)} \otimes K^{r} \otimes W^{(n)} \otimes L^{r} \\
\stackrel{\theta \otimes \theta^{\prime}}{\longrightarrow} K \otimes L
\end{aligned}
$$

Here $U$ is the evident shuffling isomorphism, $T(x \otimes y)=(-1)^{\operatorname{deg} x \operatorname{deg} y} y \otimes x$, and $\psi: W \rightarrow W \otimes W$ is any fixed $\Lambda \pi$-morphismover $\Lambda$; conditions (i) and (ii) are clearly satisfied by the pair $(\mathrm{K} \otimes L, \tilde{\theta})$. An object $(\mathrm{K}, \theta) \in \zeta(\pi, \mathrm{n}, \Lambda)$ is said to be a Cartan object if the product $K \otimes K \rightarrow K$ is a morphism in $\zeta(\pi, n, \Lambda)$. When $\pi$ is cyclic of prime order $p$, we agree to choose $W$ to be the explicit resolution $W(p, \Lambda)$ of Definition 1.2 , and we abbreviate $\zeta\left(\pi, n, z_{p}\right)$ to $\boldsymbol{\zeta}(p, n)$ and $P\left(\pi, n, z_{p}\right)$ to $P(p, n)$. An object $(K, \theta) \in \zeta(p, n)$ is said to be reduced $\bmod p$ if $(K, \theta)$ is obtained by reduction $\bmod p$ from an object $(\tilde{K}, \tilde{\theta}) \in \zeta(\pi, n, Z)$ such that $\tilde{\mathrm{K}}$ is a flat $\mathrm{Z}$-module.

We can now define the Steenrod operations in the homology $H(K)$ of an object $(K, \theta) \in \zeta(p, n)$. Observe that if $x \in H(K)$ and $0 \leq i \leq n$, then $e_{i} \otimes x^{p}$ is a welldefined element of $H\left(W^{(n)} \otimes_{\pi} K^{p}\right) \cong H\left(W^{(n)} \otimes_{\pi} H(K)^{p}\right)$ by Lemmas 1.1 and 1.3 ; here (iv) of Lemma 1.1 applies, since $\pi \subset \Sigma_{p}$ contains only even permutations, and shows that $e_{i} \otimes x^{p}$ is represented by $e_{i} \otimes a^{p} \in W^{(n)} \otimes K^{p}$ for any representative cycle a of $\mathbf{x}$.

Definitions 2.2. Let $(K, \theta) \in \zeta(p, n)$ and let $x \in H_{q}(K)$. For $0 \leq i \leq n$, define $D_{i}(x) \in H_{p q+i}(K)$ by $D_{i}(x)=\theta_{*}\left(e_{i} \otimes x\right), \theta_{*}: H\left(W^{(n)} \otimes_{\pi} K^{p}\right) \rightarrow H(K)$. If $p=2$, define $\mathrm{P}_{\mathrm{s}}: \mathrm{H}_{\mathrm{q}}(\mathrm{K}) \rightarrow \mathrm{H}_{\mathrm{q}+\mathrm{s}}(\mathrm{K})$ for $\mathrm{s} \leq \mathrm{q}+\mathrm{n}$ by the formula

$$
P_{s}(x)=0 \text { if } s<q ; P_{s}(x)=D_{s-q}(x) \text { if } s \geq q \text {. }
$$

If $p>2$, define $P_{s}: H_{q}(K) \rightarrow H_{q}+2 s(p-1)(K)$ for $2 s(p-1) \leq q(p-1)+n$ and define 
$\beta P_{s}: H_{q}(K) \longrightarrow H_{q+2 s}(p-1)-1(K)$ for $2 s(p-1) \leq q(p-1)+n+1$ by the formulas

$$
\begin{aligned}
& P_{s}(x)=0 \text { if } 2 s<q ; P_{s}(x)=(-1)^{s} v(q) D(2 s-q)(p-1)^{(x)} \text { if } 2 s \geq q \text { and } \\
& \beta P_{s}(s)=0 \text { if } 2 s \leq q ; \quad \beta P_{s}(x)=(-1)^{s} v(q) D(2 s-q)(p-1)-1 \text { (x) if } 2 s>q, \\
& \text { where } v(2 j+\varepsilon)=(-1)^{j}(m !)^{\varepsilon}, j \text { any integer, } \varepsilon=0 \text { or } 1, m=\frac{1}{2}(p-1), \\
& \text { or, equivalently since }(m !)^{2} \equiv(-1)^{m+1} \bmod p, v(q)=(-1)^{q(q-1) m / 2}(m !)^{q}
\end{aligned}
$$

Observe that, if $n=\infty$, the $P_{s}$ and, if $p>2$, the $\beta P_{s}$ are defined for all integers $s$ and that $\beta P_{s}$ is a single symbol which is not a priori related to any Bockstein operation. The $\mathrm{P}_{\mathbf{s}}$ and $\beta_{\mathbf{s}}$ a re appropriately defined for applications to homology; as shown in section 5 , the appropriate formulation for cohomology is obtained by a simple change of notation.

The following proposition contains most of the elementary properties of the $D_{i}, P_{s}$, and $\beta P_{s}$. In particular, if $p>2$, it shows that the $P_{s}$ and $\beta P_{s}$ account for all non-trivial operations $D_{i}$ and that $\beta P_{s}$ is the composition of $P_{s}$ and the Bockstein $\beta$ provided that $(K, \theta)$ is reduced mod $p$.

Proposition 2.3. Let $(K, \theta) \in \zeta(p, n)$ and consider $D_{i}: H_{q}(K) \rightarrow \underset{p q+i}{H}(K)$.

(i) If $f: K \rightarrow K^{\prime}$ is a morphism in $\zeta(p, n)$, then $D_{i \neq *} f_{*}=f_{*} D_{i}$.

(ii) If $i<n$, then $D_{i}$ is a homomorphism.

(iii) $D_{0}$ is the $p$-th power operation in the algebra $H(K)$ and if $(K, \theta)$ is unital, then $D_{i}(e)=0$ for $i \neq 0$, where $e \in H_{0}(K)$ is the identity.

(iv) If $p>2$ and $i<n$, then $D_{i}=0$ unless either

(a) $q$ is even and $i=2 t(p-1)$ or $i=2 t(p-1)-1$ for some $t$, or

(b) $q$ is odd and $i=(2 t+1)(p-1)$ or $i=(2 t+1)(p-1)-1$ for some $t$.

(v) If $(K, \theta)$ is reduced $\bmod p$ and $\beta$ is the Bockstein, then
(a) $\beta D_{2 i}=D_{2 i-1}$ if either $p>2$ or $q$ is even, and $2 i<n$
(b) $\beta D_{2 i+1}=D_{2 i}$ if $p=2$ and $q$ is odd, and $2 i+1<n$. 
Proof. Part (i) is immediate from the definitions and from Lemma 1. I (iv), and part (ii) is immediate from the definitions.

(ii) Let $a, b \in K_{q}$ be cycles and define $\Delta(a, b)=(a+b)^{p}-a^{p}-b^{p} \in K^{p} \cdot \Delta(a, b)$ is a sum of monomials involving both $\mathrm{a}^{\prime} \mathrm{s}$ and $\mathrm{b}^{\prime} \mathrm{s}$, and $\pi$ permutes such monomials freely. Let $c \in K^{\mathrm{P}}$ be a sum of monomials whose permutations under $\pi$ give each monomial of $\Delta(\mathrm{a}, \mathrm{b})$ exactly once. Then $\Delta(\mathrm{a}, \mathrm{b})=\mathrm{Nc}$. If $\mathrm{i}$ is odd, then $d\left(e_{i+1} \otimes c\right)=e_{i} \otimes N c$ and if $i$ is even, then $d\left(T^{p-2} e_{i+1} \otimes c\right)=e_{i} \otimes N c$ in $W^{(n)} \otimes_{\pi} K^{p}, i<n$, since $T^{p-1}=N$ in $z_{p} \pi$. Thus $e_{i} \otimes \Delta(a, b)$ is a boundary and therefore $D_{i}$ is a homomorphism, $i<n$.

(iv) In the notations of Definition, $\widehat{Y}_{0} 1$, we have that $\theta$ is homotopic to a composite $W^{(n)} \otimes_{\pi} K^{p} \stackrel{j \otimes 1}{\longrightarrow} V^{(n)} \otimes_{\pi} K^{p} \rightarrow V^{(n)} \otimes_{\Sigma} K^{p} \stackrel{\varphi}{\longrightarrow} K$. Since nothing is changed by tensoring with two copies of $z_{p}(q)$, this composite can equally well be written as

$$
W^{(n)}(q) \otimes_{\pi} K^{p}(q) \stackrel{j \otimes_{1}}{\longrightarrow} V^{(n)}(q) \otimes_{\Sigma} K^{p}(q) \stackrel{\phi}{\longrightarrow} K
$$

Let $a \in K_{q}$ be a cycle. Then, bythe definition of $K^{p}(q), a^{p}$ is a basis for a trivial $\Sigma_{p}$-subcomplex of $K^{P}(q)$. Therefore, if $j\left(e_{j}\right)=d(f)$ in $v^{(n)} \otimes_{\Sigma_{p}} z_{p}(q)=V^{(n)}(q) \otimes_{\Sigma} z_{p}$, then $d\left(f \otimes a^{p}\right)=j\left(e_{i}\right) \otimes_{a^{p}}$ in $V^{(n)}(q) \otimes_{\Sigma} K^{p}(q)$. For $i<n, j$ induces $j_{*}: H_{i}\left(\pi ; z_{p}(q)\right) \rightarrow H_{i}\left(\Sigma ; ; Z_{p}(q)\right)$, and the desired conclusion now follows immediately from Lemma 1.4.

(v) Let $(K, \theta)$ be the mod $p$ reduction of $(\tilde{K}, \tilde{\theta})$. Let a $\in \tilde{K}_{q}$ satisfy $d(a)=p b$. An easy calculation demonstrates that, in $\tilde{\mathrm{K}}^{\mathrm{p}}$,

$$
\begin{aligned}
& d\left(a^{p}\right)=p^{p-1} \text { if } p>2 \text { or } q \text { is even; } \\
& d\left(a^{2}\right)=2 \text { Tab if } p=2 \text { and } q \text { is odd. }
\end{aligned}
$$

In the former case, if $2 i<n$, then, in $W(p, z)^{(n)} \otimes_{\pi} \widetilde{K}^{p}$,

$$
\begin{aligned}
\mathrm{d}\left(\mathrm{e}_{2 \mathrm{i}} \otimes \mathrm{a}^{\mathrm{p}}\right) & =\mathrm{e}_{2 \mathrm{i}-1} \otimes \mathrm{Na}^{\mathrm{p}}+\mathrm{pe}_{2 \mathrm{i}} \otimes \mathrm{Nba}^{\mathrm{p}-1} \\
& \equiv \mathrm{p}\left[\mathrm{e}_{2 \mathrm{i}-1} \otimes \mathrm{a}^{\mathrm{p}}+\mathrm{d}\left(\mathrm{T}^{\mathrm{p}-2} \mathrm{e}_{2 \mathrm{i}+1} \otimes \mathrm{ba}{ }^{\mathrm{p}-1}\right)\right] \bmod \mathrm{p}^{2},
\end{aligned}
$$

since $T^{p-1} \equiv N \bmod p$. In the latter case, if $2 i+1<n$, then

$$
\mathrm{d}\left(\mathrm{e}_{2 \mathrm{i}+1} \otimes \mathrm{a}^{2}\right)=\mathrm{e}_{2 \mathrm{i}} \otimes \mathrm{Ta}^{2}-2 \mathrm{e}_{2 \mathrm{i}+1} \otimes \mathrm{Tab} \equiv 2\left[\mathrm{e}_{2 \mathrm{i}} \otimes \mathrm{a}^{2}-\mathrm{d}\left(\mathrm{e}_{2 \mathrm{i}+2} \otimes \mathrm{ab}\right)\right] \bmod 4 .
$$


Thus, in $\mathrm{H}\left(\mathrm{W}\left(\mathrm{p}, \mathrm{z}_{\mathrm{p}}\right)^{(\mathrm{n})} \otimes_{\pi} \mathrm{K}^{\mathrm{p}}\right)$, if $\bar{a}$ is the $\bmod \mathrm{p}$ reduction of $a$, then $\beta\left\{e_{2 i} \otimes \bar{a}^{p}\right\}=\left\{e_{2 i-1} \otimes \bar{a}^{p}\right\}$ in case $(a)$ and $\beta\left\{e_{2 i+1} \otimes \bar{a}^{2}\right\}=\left\{e_{2 i} \otimes \bar{a}^{2}\right\}$ in case (b). Since $\theta$ is the mod $p$ reduction of the $\operatorname{map} \theta: W(p, z)^{(n)} \otimes_{\pi} K^{p} \rightarrow K, \beta \theta_{* *}=\theta_{* *} \beta$, and the result follows.

Of course, (i) and (ii) imply that the $\mathrm{P}_{s}$ and $\beta \mathrm{P}_{s}$ are natural homomorphisms (except, if $\mathrm{n}<\infty$, for the last operation). A check of constants gives the following corollary of part (iii).

Corollary 2.4. Let $(K, \theta) \in \zeta(p, n)$. Then $P_{q}(x)=x^{p}$ if $p=2$ and $x \in H_{q}(K)$ or if $p>2$ and $x \in \mathrm{H}_{2 q}(\mathrm{~K})$. If $(K, \theta)$ is unital, then $\mathrm{P}_{s}(e)=0$ for $s \neq 0$.

The implications of (iv) and ( $v$ ) for the $P_{s}$ and $\beta P_{S}$ are clear if $p>2$. If $p=2$, we have the following corollary of $(v)$.

Corollary 2.5. If $(K, \theta) \in \zeta(2, \infty)$ is reduced mod 2, then $\beta P_{s+1}=s P_{s}$.

The following result is the external Cartan formula.

Proposition 2.6. Let $(K, \theta)$ and $\left(L, \theta^{\prime}\right)$ be objects of $\zeta(p, n)$. Let $x \in \mathrm{H}_{q}(K)$ and $y \in \mathrm{H}_{\mathbf{x}}(L)$. Consider $x \otimes y \in H(K) \otimes H(L)=H(K \otimes L)$.

(i) If $p=2$, then $D_{i}(x \otimes y)=\sum_{j+k=i} D_{j}(x) \otimes D_{k}(y)$ for $i \leq n$.

(ii) If $p>2$, then $D_{2 i}(x \otimes y)=(-1)^{\mathrm{mqr}} \sum_{j+k=i} D_{2 j}(x) \otimes D_{2 k}(y)$ for $2 i \leq n$, and

$$
\begin{gathered}
D_{2 i+1}(x \otimes y)=(-1)^{m} \sum_{j+k=i}\left(D_{2 j+1}(x) \otimes D_{2 k}(y)+(-1)^{q} D_{2 j}(x) \otimes D_{2 k+1}(y)\right) \quad \text { for } \\
2 i+1 \leq n .
\end{gathered}
$$

Proof. By Lemmas 1.1 and 1.3, we may work in $W^{(n)} \otimes_{\pi}[H(K) \otimes H(L)]^{p}$. Since $\pi$ operates trivially on $(x \otimes y)^{\mathrm{P}}$, we may compute $\theta_{*}\left(e_{j} \otimes(x \otimes y)^{\mathrm{P}}\right)$ by means of the induced coproduct on $W^{(n)} \otimes_{\pi} Z_{p}$, as given in (2) of Definition 1.2. The result follows by direct calculation from

$$
\theta_{*}\left(e_{i} \otimes(x \otimes y)^{p}\right)=\left(\theta_{*} \otimes \theta_{* k}^{\prime}\right)(1 \otimes T \otimes 1)(\psi \otimes U)\left(e_{i} \otimes(x \otimes y)^{p}\right) .
$$


A trivial ve rification of constants, together with part (iv) of Proposition 2.3, yields the following corollary.

Corollary 2.7. Let $(K, \theta)$ and $\left(L, \theta^{\prime}\right)$ be objects of $\zeta(p, n)$. Let $x \in H_{q}(K)$ and $y \in H_{r}(L)$. Then $P_{s}(x \otimes y)=\sum_{i+j=s} P_{i}(x) \otimes P_{j}(y)$ and, if $p>2$, $\beta P_{s+1}(x \otimes y)=\sum_{i+j=s}\left(\beta P_{i+1}(x) \otimes P_{j}(y)+(-1)^{q} P_{i}(x) \otimes \beta P_{j+1}(y)\right)$.

Of course, if $(K, \theta)$ is a Cartan object in $\zeta(p, n)$, then the corollary and the naturality of the operations imply that the $P_{s}$ and, if $p>2$, the $\beta P_{s}$ on $H(K)$ satisfy the internal Cartan formulas

(1)

$$
P_{s}(x y)=\sum_{i+j=s} P_{i}(x) P_{j}(y) \quad \text { and }
$$

$$
\beta P_{s+1}(x y)=\sum_{i+j=s}\left(\beta P_{i+1}(x) P_{j}(y)+(-1){ }^{\operatorname{deg} x_{P}} P_{i}(x) \beta P_{j+1}(y)\right)
$$




\section{Chain level operations, suspension, and spectral sequences}

In this section, we define chain level Steenrod operations and use them to prove that the homology operations commute with suspension. The chain level operations can also be used to study the behavior of Steenrod operations in spectral sequences and, in particular, we shall prove a general version of the Kudo transgression theorem.

Theorem 3.1. Let $(K, \theta) \in \zeta(p, \infty)$. Then there exist functions $\mathrm{P}_{\mathrm{s}}: \mathrm{K}_{\mathrm{q}} \rightarrow \mathrm{K}_{\mathrm{q}+\mathrm{s}}$ if $\mathrm{p}=2$ and $\mathrm{P}_{\mathrm{s}}: \mathrm{K}_{\mathrm{q}} \rightarrow \mathrm{K}_{\mathrm{q}+2 \mathrm{~s}(\mathrm{p}-1)}$ and $\beta \mathrm{P}_{\mathrm{s}}: \mathrm{K}_{\mathrm{q}} \rightarrow \mathrm{K}_{\mathrm{q}+2 \mathrm{~s}(\mathrm{p}-1)-1}$ if $p>2$ which satisfy the following properties.

(i) $\quad \mathrm{dP}_{\mathrm{s}}=\mathrm{P}_{\mathrm{s}} \mathrm{d}$ and $\quad \mathrm{d} \beta \mathrm{P}_{\mathrm{s}}=-\beta \mathrm{P}_{\mathrm{s}} \mathrm{d}$

(ii) If a is a cycle which represents $x \in H(K)$, then $P_{s}(a)$ and $\beta P_{s}(a)$ are cycles which represent $\mathrm{P}_{\mathbf{S}}(\mathrm{x})$ and $\beta_{\mathrm{S}}(\mathrm{x})$.

(iii) If $f:(K, \theta) \rightarrow\left(K^{\prime}, \theta^{\prime}\right)$ is a morphism in $P(p, \infty)$, so that $f \theta=\theta^{\prime}\left(1 \otimes f^{p}\right)$, then $f P_{s}=P_{s} f$ and $f \beta P_{s}=\beta P_{s} f$.

Proof. Let $a \in K_{q}$ and write $b=d(a) \in K_{q-1}$. In the case $p=2$, define (1) $P_{s}(a)=\theta(c)$, whe re $c=e_{s-q+1} \otimes b \otimes a+e_{s-q} \otimes a \otimes a \in W \otimes_{\pi} K^{2}$.

The verification of $(i),(i i)$, and (iii) is trivial. Thus assume that $p>2$. Let $(a, b)$ denote the subcomplex of $K$ with basis $a$ and $b$, so that $(a, b)^{p} \subset K^{p}$. Define $s:(a, b) \rightarrow(a, b)$, of degree one, by $s(a)=0$ and $s(b)=a$. Then $d s+s d=1$ on $(a, b)$. Let $S=1^{p-1} \otimes s$ on $(a, b)^{p}$. Then $d S+S d=1$ on $(a, b)^{p}$ and $S$ is given

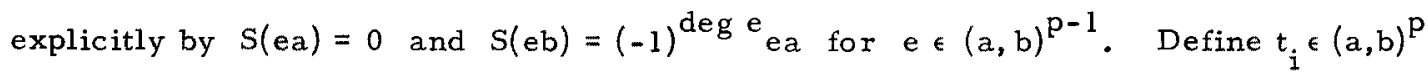
for $0 \leq i \leq p$ by the inductive formula

(2) $t_{0}=b^{p} ; t_{1}=b^{p-1} a ; t_{2 k}=S\left(\alpha^{-1} t_{2 k-1}-t_{2 k-1}\right) ; t_{2 k+1}=S\left(N t_{2 k}\right)$.

Since $d S+S d=1$, an easy calculation demonstrates that

(3) $d\left(t_{1}\right)=t_{0} ; d\left(t_{2 k}\right)=\left(\alpha^{-1}-1\right) t_{2 k-1}$ and $d\left(t_{2 k+1}\right)=N t_{2 k}, 1 \leq k \leq m$.

A straightforward induction, which uses the explicit formula for $S$, yields 
(4) $\quad t_{2 k}=\sum_{I}(-1)^{k q}(k-1) ! b^{i_{1}} a^{2} b^{i_{2}} a^{2} \ldots b^{i_{k}}{ }^{2}, 1 \leq k \leq m$, summed over all k-tuples $I=\left(i_{1}, \ldots, i_{k}\right)$ such that $\sum i_{j}=p-2 k$; and

(5) $\quad t_{2 k+1}=\sum_{I}(-1)^{k q}{ }_{k !} b^{i_{1}} a^{2} \ldots b^{i_{k}} a^{2} b^{i_{k+1}} a, 0 \leq k \leq m$, summed over all $(k+1)$-tuples $I=\left(i_{1}, \ldots, i_{k+1}\right)$ such that $\sum i_{j}=p-1-2 k$.

In particular, $t_{p}=t_{2 m+1}=(-1)^{m q} m ! a^{p}$ (since each $\left.i_{j}=0\right)$. Now let $j=(2 s-q+1)(p-1)$ and define chains $c$ and $c^{\prime}$ in $W \otimes_{\pi} K^{p}$ by the following formulas (where, by convention, $e_{i}=0$ if $i<0$ ):

(6) $\quad c=\sum_{k=0}^{m}(-1)^{k} e_{j-2 k} \otimes t_{2 k+1}-\sum_{k=1}^{m}(-1)^{k} e_{j+1-2 k} \otimes\left(\alpha^{-1}-1\right)^{p-2} t_{2 k}$;

(7) $\quad c^{\prime}=\sum_{k=0}^{m}(-1)^{k} e_{j-1-2 k} \otimes t_{2 k+1}+\sum_{k=1}^{m}(-1)^{k} e_{j-2 k} \otimes t_{2 k}$.

Then an easy computation, which uses Definition 1.2 and (3), gives

$$
d(c)=e_{j} \otimes b^{p} \quad \text { and } \quad d\left(c^{\prime}\right)=-e_{j-1} \otimes b^{p} \quad(j=(2 s-q+1)(p-1))
$$

In calculating $\mathrm{d}(\mathrm{c})$, the salient observations are that $\mathrm{Nt}_{\mathrm{p}}=0$, that $\alpha e_{i} \otimes t=e_{i} \otimes \alpha^{-1} t$ for $t \in K^{p}$ by the very definition of a tensor product, and that $\left(\alpha^{-1}-1\right)^{\mathrm{p}-1}=\mathrm{N}$ in $\mathrm{z}_{\mathrm{p}} \pi$. Finally, define

(9) $\quad P_{s}(a)=(-1)^{s} v(q-1) \theta(c)$ and $\beta P_{s}(a)=(-1)^{s} v(q-1) \theta\left(c^{\prime}\right)$.

If $a$ is a cycle, $b=0$, then $t_{i}=0$ for $i<p$ and $t_{p}=(-1)^{m q} m: a^{p}$, hence

$$
c=(-1)^{m(q+1)} m ! e_{(2 s-q)(p-1)} \otimes a^{p} \text { and } c^{\prime}=(-1)^{m(q+1)} m ! e_{(2 s-q)(p-1)-1} \otimes a^{p} \text {. }
$$

It is easy to verify that $v(q)=(-1)^{\mathrm{m}(\mathrm{q}+1)} \mathrm{m} ! v(\mathrm{q}-1)$ and now (ii) is obvious from (9) and (10) and (i) follows from (8), (9), and (10), applied to the cycle $b \in K_{q-1}$. Part (iii) is immediate from (9).

The remaining results of this section are corollaries of the theorem and its proof. We first define and study a very general notion of suspension. 
Definition. Let $f: K^{\prime} \rightarrow K$ and $g: K \rightarrow K^{\prime \prime}$ be morphisms of $\Lambda$-complexes such that $g f=0$. Define $\sigma: \operatorname{Ker} f_{*} \rightarrow$ Coker $g_{*}$ by the formula $\sigma\left\{b^{\prime}\right\}=\{g(a)\}$, where $b^{\prime}$ represents $\left\{b^{\prime}\right\} \in \operatorname{Ker} f_{*^{\prime}}$ and $d(a)=f\left(b^{\prime}\right)$ in $K$. It is trivial to verify that $\sigma$ is well-defined, and we call $\sigma$ the suspension.

We can now prove that the $P_{s}$ commute with suspension. We remark that if $n=\infty$, the hypotheses of the next theorem simplify to the requirement that $f$ and $g$ be morphisms in $\mathcal{P}(\mathrm{p}, \infty)$ such that $\mathrm{gf}=0$. For $\mathrm{n}<\infty$, the stated hypotheses arise in practice in the study of iterated loop spaces.

Theorem 3.3. Let $\left(K^{\prime}, \theta^{\prime}\right) \in \zeta(p, n+1)$ and let $\left(K^{\prime \prime}, \theta^{\prime \prime}\right) \in \zeta(p, n)$. Let $\mathrm{K}$ be a $\mathrm{Z}_{\mathrm{p}}$-complex and let $\mathrm{f}: \mathrm{K}^{\prime} \rightarrow \mathrm{K}$ and $\mathrm{g}: \mathrm{K} \rightarrow \mathrm{K}^{\prime \prime}$ be morphisms of complexes such that $g f=0$. Define a subcomplex $\widetilde{K}$ of $W^{(n+1)} \otimes K^{p}$ by

$$
\widetilde{K}=W^{(n+1)} \otimes f\left(K^{\prime}\right)^{p}+\bar{W}^{(n+1)} \otimes f\left(K^{\prime}\right)^{p-1} \otimes K+W^{(n)} \otimes K^{p},
$$

where $\bar{W}^{(n+1)}=W^{(n)} \oplus z_{p} e_{n+1}$ (that is, $e_{n+1} \in \bar{W}^{(n+1)}$ but $\alpha^{i} e_{n+1} \notin \bar{W}^{(n+1)}$ for $i \leq i<p$ ). Suppose given a $\pi$-morphism $\theta: \widetilde{K} \rightarrow K$ (where, by convention, $\pi$ does not act on $\left.e_{n+1} \otimes f\left(K^{1}\right)^{p-1} \otimes K\right)$ such that the following diagram is commutative:

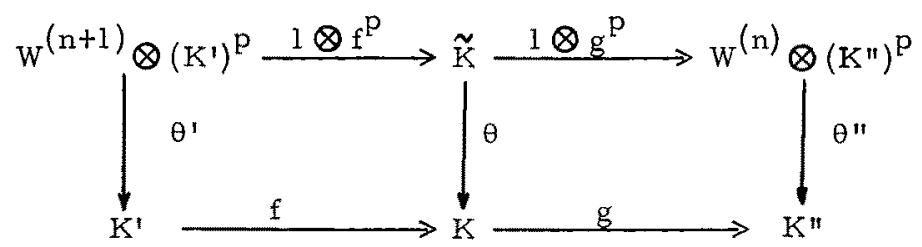

(Here $g f=0$ ensures that $\left.\left(1 \otimes g^{p}\right)(\tilde{K}) \subset W^{(n)} \otimes\left(K^{\prime \prime}\right)^{p}.\right)$ Observe that Ker $f_{*}$ is closed under the $P_{S}$ and $\beta P_{S}$ and that there are well-defined induced $P_{S}$ on Coker $g_{*}$. Let $x \in \operatorname{Ker} f_{*^{*}}$ Then $\sigma P_{s}(x)=P_{s} \sigma(x)$ and $\sigma \beta P_{s}(x)=-\beta P_{s} \sigma(x)$ whenever $\mathrm{P}_{\mathrm{s}}(\mathrm{x})$ and $\beta \mathrm{P}_{\mathrm{s}}(\mathrm{x})$ are defined.

Proof. Let $\operatorname{deg}(x)=q-1$ and let $b^{\prime} \in K^{\prime}$ represent $x$. Let $b=f\left(b^{\prime}\right)$ and let $d(a)=b$ in $K$, so that $g(a)$ represents $\sigma(x)$. The hypothesis guarantees that if $s$ is such that $P_{s}(x)$ or $\beta P_{s}(x)$ is defined, then the chain level operation $P_{s}(a)$ or $\beta P_{S}(a)$ constructed in the previous proof is also defined. Of course, this is 
clear if $\mathrm{n}=\infty$; if $\mathrm{n}<\infty$, we need only verify that all elements involved in the definition of $\mathrm{P}_{\mathrm{S}}(\mathrm{a})$ or $\beta \mathrm{P}_{\mathrm{S}}(\mathrm{a})$ are present in $\tilde{\mathrm{K}}$. For example, if $\mathrm{p}=2$, the last operation $\mathrm{P}_{\mathrm{s}}(\mathrm{x})$ occurs for $\mathrm{s}=\mathrm{q}+\mathrm{n}$ and then $\mathrm{P}_{\mathrm{s}}(\mathrm{a})=\theta(\mathrm{c})$, where $c=e_{n+1} \otimes b \otimes a+e_{n} \otimes a \otimes a$, and $c$ is indeed in $\widetilde{K}$. Now our diagram ensures that $\operatorname{fP}_{S}\left(b^{\prime}\right)=P_{S} f\left(b^{\prime}\right)$, hence $f P_{S}\left(b^{\prime}\right)=d P_{s}(a)$, and that $g P_{s}(a)=P_{s} g(a)$. $\sigma P_{S}(x)=P_{S} \sigma(x)$ follows from the definition of $\sigma$, and the proof that $\sigma \beta P^{s}(x)=$ $-\beta P_{s} \sigma(x)$ is equally simple.

Note that if $p>2$ and all objects are reduced $\bmod p$, then $\sigma \beta=-\beta \sigma$, which is consistent with the theorem. The theorem implies that $\sigma\left(x^{p}\right)=0$ and that $\sigma \beta P_{s}(x)=0$ if $p>2$ and $\operatorname{deg}(x)=2 s-1$; if $\left(K^{\prime \prime}, \theta^{\prime \prime}\right)$ is reduced mod $p$, the latter statement becomes $\beta \sigma(x)^{\mathrm{P}}=0$. The operation $\beta \mathrm{P}_{\mathrm{s}}(\mathrm{x})$, deg $(\mathrm{x})=2 \mathrm{~s}-1$, plays a special role in many applications; the following very useful technical result about about this operation is known as the Kudo transgression theorem. It applies to the Dyer-Lashof operations in the homology Serre spectral sequence of the path-space fibration $\Omega^{n} \mathrm{X} \rightarrow \mathrm{P} \Omega^{\mathrm{n}-1} \mathrm{X} \rightarrow \Omega^{\mathrm{n}-1} \mathrm{X}$, to the Steenrod operations in the cohomology Serre spectral sequence of a fibration $\mathrm{F} \rightarrow \mathrm{E} \rightarrow \mathrm{B}$ (with $\mathrm{K}^{\prime} \rightarrow \mathrm{K} \rightarrow \mathrm{K}^{\prime \prime}$ being $C^{*}(B) \rightarrow C^{*}(E) \rightarrow C^{*}(F)$, graded by subscripts) and to the spectral sequence of Adams $[1, p .210]$ for cocommutative Hopf algebras.

Theorem 3.4. Assume, in addition to the hypotheses of Theorem 3.3, that $K$ has an increasing fitration $\left\{F_{i} K\right\}$, that $H_{o}\left(K^{\prime}\right)=Z_{p}=H_{o}\left(K^{\prime \prime}\right)$, and that there is a morphism of complexes $\pi: K \otimes f\left(K^{3}\right) \rightarrow K$ such that either

(i) $K^{\prime}, K$, and $K^{\prime \prime}$ are positively graded, $F_{i} K=0$ if $i<0, F_{i} K_{i}=K$ if $i \geq 0$, $f\left(K^{\prime}\right) \subset F_{0} K, \pi\left(F_{i} K \otimes f\left(K^{\prime}\right)\right) \subset F_{i} K$, and $f$ and $g$ induce isomorphisms $E^{2} f: H_{j}\left(K^{\prime}\right) \rightarrow E_{o j}^{2} K$ and $E^{2} g: K \rightarrow H_{i}\left(K^{\prime \prime}\right)$ and $\pi$ induces a morphism $E^{2} \pi: E_{i j}^{2} K \otimes E_{o k}^{2} K \rightarrow E_{i, j+k}^{2} K$ such that the composite morphism $E^{2} \pi\left[\left(E^{2} g\right)^{-1} \otimes E^{2} f\right]: H_{i}\left(K^{\prime \prime}\right) \otimes H_{j}\left(K^{\prime}\right) \rightarrow E_{i j}^{2} K$ is an isomorphism; or 
(ii) $K^{\prime}, K$, and $K^{\prime \prime}$ are negatively graded, $F_{i} K=K$ if $i \geq 0, F_{i-1} K_{i}=0$ if $i \leq 0$, $f\left(K_{i}\right) \subset F_{i} K, \pi\left(F_{i} K \otimes f(K !)\right) \subset F_{i+j} K$, and $f$ and $g$ induce isomorphisms $E^{2} f: H_{i}\left(K^{\prime}\right) \rightarrow E_{i, o}^{2} K$ and $E_{g}^{2}: E_{o j}^{2} K \rightarrow H_{j}\left(K^{\prime \prime}\right)$ and $\pi$ induces a morphism $E^{2} \pi: E_{i j}^{2} K \otimes E_{k o}^{2} K \rightarrow E_{i+k, j}^{2} K$ such that the composite morphism $E^{2} \pi\left[\left(E^{2} g\right)^{-1} \otimes E^{2} f\right]: H_{j}\left(K^{\prime \prime}\right) \otimes H_{i}\left(K^{\prime}\right) \rightarrow E_{i j}^{2} K$ is an isomorphism.

Let $\tau$ be the transgression, $\tau=d_{t}: E_{\text {to }}^{t} K \rightarrow E_{0, t-1}^{t} K$ in (i) and $\tau=d_{1-t}: E_{\text {ot }}^{1-t} K \rightarrow E_{t-1, o}^{1-t} K \quad(t<0)$ in (ii). Then $\tau$ is the inverse additive relation to $\sigma$, and if $y \in H_{q}\left(K^{\prime \prime}\right)$ transgresses to $x \in H_{q-1}\left(K^{\prime}\right)$, then $P_{s}(y)$ and if $\mathrm{p}>2, \quad \beta \mathrm{P}_{\mathrm{s}}(\mathrm{y})$ transgresses to $\mathrm{P}_{\mathrm{s}}(\mathrm{x})$ and $-\beta \mathrm{P}_{\mathrm{S}}(\mathrm{x})$, whenever the operations are defined. Moreover, if $\mathrm{p}>2$ and $\mathrm{q}=2 \mathrm{~s}$, then $\mathrm{y}^{\mathrm{p}-1} \otimes \mathbf{x}$ transgresses to $-\beta P_{s}(x)$ (that is, $d_{q(p-1)}\left(y^{p-1} \otimes x\right)=-\beta P_{s}(x)$ in case $(i)$ and $\mathrm{d}_{1-\mathrm{q}(\mathrm{p}-1)}\left(\mathrm{y}^{\mathrm{p}-1} \otimes \mathrm{x}\right)=-\beta \mathrm{P}_{\mathrm{s}}(\mathrm{x})$ in case (ii) provided that

(iii) if $a_{j} \in F_{i_{j}} K$, then $\theta\left(e_{k} \otimes a_{1} \otimes \ldots \otimes a_{p}\right) \in F_{i} K, i=\sum i_{j}+k$, and

(iv) The restriction of $\theta$ to $e_{0} \otimes K^{p-1} \otimes f\left(K^{\prime}\right)$ induces a morphism $E^{2} \theta:\left(E_{*_{0}}^{2} K\right)^{p-1} \otimes E_{0 *}^{2} K \rightarrow E^{2} K$ in $(i)$ and $E^{2} \theta:\left(E_{0 *}^{2} K\right)^{p-1} \otimes E_{* 0}^{2} K \rightarrow E^{2} K$ in (ii) such that $E^{2} \theta=E^{2} \pi\left[\left(E^{2} g\right)^{-1} \phi\left(E^{2} g\right)^{p-1} \otimes 1\right]$, where $\phi: H\left(K^{\prime \prime}\right)^{P-1} \rightarrow H\left(K^{\prime \prime}\right)$ is the iterated product.

Proof. Let $y \in \mathrm{H}_{\mathrm{q}}\left(\mathrm{K}^{\prime \prime}\right)$. By the definition of the differentials in the spectral sequence of a filtered complex, $T(y)$ is defined if and only if $y$ is represented by $g(a)$ for some $a \in K_{q}$ such that $d(a)=f\left(b^{\prime}\right)$ for some cycle $b^{\prime} \in K_{q-I}^{\prime}$, and then $x=\tau(y)=\left\{b^{\prime}\right\}$. Thus the first statement follows from the properties of the chains $\mathrm{P}_{\mathrm{S}}(\mathrm{a})$ and $\beta \mathrm{P}_{\mathrm{S}}(\mathrm{a})$. For the second statement, consider $\beta P_{s}(a), q=2 s$. Since $d \beta P_{s}(a)=-\beta P_{s} f\left(b^{\prime}\right), a$ and $b^{\prime}$ as above, it suffices to prove that $\beta P_{s}(a)$ represents $y^{p-1} \otimes x$ in $E^{2} K . \quad \beta P_{S}(a)=-m ! \theta\left(c^{\prime}\right)$ by $(9)$ of the proof of Theorem 3.1 and the observation that $v(\mathrm{q}-1)=v(2 \mathrm{~s}-1)=(-1)^{\mathrm{s}-1} \mathrm{~m} !$. In the definition (7) of $c^{\prime}$, the term with $k=m$ in the first sum involves $e_{-1}$ (since 
$q=2 s$ implies $j=p-1$ ) and is therefore zero. The term with $k=m$ in the second sum is $(-1)^{m} e_{0} \otimes t_{p-1}$ where, by (4), $t_{p-1}=(m-1) ! \sum_{i=0}^{m-1} a^{2 i} b a^{2(m-i)}$, $b=d(a)$. It is easy to see that

$$
\sum_{i=0}^{m-1} a^{2 i_{b a}}{ }^{2(m-i)}=P(\alpha) a^{p-1} b, \text { where } P(\alpha)=\sum_{i=1}^{m} \alpha^{2 i-1},
$$

and direct calculation shows that $P(\alpha)=\mathrm{m}+\mathrm{Q}(\alpha)$, in $\mathrm{Z}_{\mathrm{p}} \pi$, where $Q(\alpha)=\sum_{j=1}^{m} j\left(\alpha^{2 j}+\alpha^{2 j+1}\right)$. Let $c^{\prime \prime}=(-1)^{m}(m-1) ! e_{1} \otimes Q(\alpha) a^{p-1} \otimes b \in W \otimes K^{p}$. Then $c^{\prime}-d\left(c^{\prime \prime}\right)=(-1)^{m} m ! e_{0} \otimes a^{p-1} b$ plus a linear combination of terms $e_{j} \otimes g$ such that $g$ has $i+1$ factors $b$ and $p-i-1$ factors a. Condition (iii) ensures that each $\theta\left(e_{i} \otimes g\right)$ has lower filtration than does $\theta\left(e_{o} \otimes a^{p-1} b\right)$ and condition (iv) ensures that $\theta\left(e_{0} \otimes a^{p-1} b\right)$ represents $y^{p-1} \otimes x \in E^{2} K$. Since $\beta P_{s}(a)$ and $-m ! \theta\left(c^{\prime}-d\left(c^{\prime \prime}\right)\right)=\theta\left(e_{0} \otimes a^{p-1} b\right)$ represent the same element of $E^{2} K$, the proof is complete.

The following proposition gives a general prescription for the study of Steenrod operations in spectral sequences; it will be useful in the study of the cohomology of the Steenrod algebra in [18]. In the applications, the determination of the function $f$ is often quite difficult and depends on how the given $\theta$ was constructed.

Proposition 3.5. Let $(K, \theta)$ be an increasingly filte red object of $\zeta(p, \infty)$ Suppose given a function $f(i, j, k)$ such that

(i) If $a_{t} \in F_{i_{t}} K_{i_{t}+j_{t}}$, where $\sum i_{t}=i$ and $\sum j_{i}=j$, then

$$
\theta\left(e_{k} \otimes a_{1} \otimes \ldots \otimes a_{p}\right) \in F_{f(i, j, k)} K_{i+j+k} ;
$$

(ii) $f(i, j, k)>f(i-r, j+r-1, k+1), \quad r \geq l$; and

(iii) $\quad f(i, j, k) \geq r+f(i-p r, j+p(r-1), k+p-1), \quad r \geq 1$.

Let $y \in E_{i j}^{r} K$. Then there exist elements $P_{s}(y) \in E_{k \ell}^{t} K$ and, if $p>2$, $\beta P_{s}(y) \in E_{k^{\prime} \ell^{\prime}}^{t^{\prime}}$ such that $d^{t} P_{s}(y)=P_{s} d^{r}(y)$ and $d^{t^{\prime}} \beta P_{s}(y)=-\beta P_{s} d^{r}(y)$, where 
(iv) If $p=2$, then $k=f(2 i, 2 j, s-i-j), \ell=i+j+s-k$, and $t=k-f(2 i-2 r, 2 j+2(r-1), s+1-i-j)$,

(v) If $p>2$, then $k=f(p i, p j,(2 s-i-j)(p-1)), \quad \ell=i+j+2 s(p-1)-k$, and $t=k-f(p i-p r, p j+p(r-1),(2 s+1-i-j)(p-1))$.

(vi) If $p>2$, then $k^{\prime}=f(p i, p j,(2 s-i-j)(p-1)-1), \ell^{\prime}=i+j+2 s(p-1)-1-k$, and $t^{\prime}=k^{\prime}-f(p i-p r, p j+p(r-1),(2 s+1-i-j)(p-1)-1)$.

Proof. Let $a \in F_{i} K_{i+j}$ represent $y$ and let $b=d(a) \in F_{i-r} K_{i+j-1}$. Consider the chain $\mathrm{P}_{\mathrm{S}}(\mathrm{a})$ constructed in Theorem 3.1. By (ii), all summands of $\mathrm{P}_{\mathrm{s}}(\mathrm{a})$ other than that involving $\mathrm{e}_{\mathrm{n}} \otimes \mathrm{a}^{\mathrm{p}}$ (for the appropriate $\mathrm{n}$ ) have lowe $\mathrm{r}$ filtration than $k$ and, by $(i), \theta\left(e_{n} \otimes a^{p}\right)$ has filtration $k$. Since $d F_{s}(b)$, where $P_{s}(b) \in F_{k-t} K$ by $(i)$ and $k-t \geq r$ by (iii), the statement about $P_{s}(y)$ follows. The proof for $\beta P_{S}(y)$ is similar.

\section{The Adem relations}

We here show that the Adem relations are valid for the Steenrod operations in $H(K)$ if $(K, \theta) \in \zeta(p, \infty)$ satisfies certain hypotheses. The general algebraic context is distinctly advantageous in the proof. We are able to exploit a trick (Lemma 4.3) used by Adem to prove the classical Adem relations, and this trick would not be available in a categorical approach to Steenrod operations since it depends on the usage of objects of $6(p, \infty)$ which are not present in many categories of interest, such as infinite loop spaces and cocommutative Hopf algebras.

We require some notations and definitions before we can proceed to the proof.

Let $\Sigma_{p^{2}}$ act as permutations on $\{(i, j) \mid 1 \leq i \leq p, 1 \leq j \leq p\}$. Embed $\pi$ in $\Sigma_{p^{2}}$ by letting $\alpha(i, j)=(i+1, j)$. Define $\alpha_{i} \in \Sigma_{p}{ }^{2}, \quad 1 \leq i \leq p$, by 
$\alpha_{i}(i, j)=(i, j+1)$ and $\alpha_{i}(k, j)=(k, j)$ for $k \neq i$, and let $\beta=\alpha_{1} \cdots \alpha_{p}$ so that $\beta(i, j)=(i, j+1)$. Then

(1) $\quad \alpha \alpha_{i}=\alpha_{i+1} \alpha ; \alpha_{i} \alpha_{j}=\alpha_{j} \alpha_{i} ;$ and $\alpha \beta=\beta \alpha$.

Let $\alpha_{i}$ generate $\pi_{i}$ and $\beta$ generate $\nu$, so that $\pi_{i}$ and $\nu$ are cyclic of order $p$. Let $\sigma=\pi \nu$ and let $T$ be generated by the $\alpha_{i}$ and $\alpha$. Then $\sigma \subset \tau, T$ is a p-Sylow subgroup of $\Sigma_{p^{2}}$, and $\tau$ is a split extension of $\pi_{1} \ldots \pi_{p}$ by $\pi_{\text {. }}$

Let $W_{1}=W$ and $W_{2}=W$ regarded, respectively, as $\pi$-free and $\nu$-free resolutions of $Z_{p}$. Let $v$ operate trivially on $W_{1}$, let $\pi$ operate trivially on $W_{2}$, and let $\sigma$ operate diagonally on $W_{1} \otimes W_{2}$. Then $W_{1} \otimes W_{2}$ is a $\sigma-$ free resolution of $\mathrm{Z}_{\mathrm{p}}$

If $M$ is any $\nu$-module, let $\tau$ operate on $M^{p}$ by letting $\alpha$ operate by cyclic permutation and by letting $\alpha_{i}$ operate on the $i$-th factor $M$ as does $\beta$. Let $\alpha_{i}$ operate trivially on $W_{1}$. Then $\tau$ operates on $W_{1}$ and we let $\tau$ operate diagonally on $W_{1} \otimes M^{p}$. In particular, $W_{1} \otimes W_{2}^{p}$ is then a $\tau$-free resolution of $Z_{p}$. Let $\mathrm{K}$ be any $\mathrm{Z}_{\mathrm{p}}$-complex. We let $\Sigma_{\mathrm{p}^{2}}$ operate on $\mathrm{K}^{\mathrm{p}^{2}}$ by permutations with the $(i, j)$-th factor $K$ being the $j$-th factor $K$ in the $i$-th factor $K^{P}$ of $K^{p^{2}}=\left(K^{p}\right)^{p}$. We let $v$ operate in the standard fashion on $w_{2} \otimes K^{p} \quad(\beta$ acting as cyclic permutation on $\mathrm{K}^{\mathrm{P}}$ ). By the previous paragraph, this fixes an operation of $T$ on $W_{1} \otimes\left(W_{2} \otimes K^{p}\right)^{p}$.

Let $Y$ be any $\Sigma_{p^{2}}$-free resolution of $Z_{p}$ and let $w: W_{1} \otimes W_{2}^{P} \rightarrow Y$ be any $T$-morphism over $Z_{p^{*}} w$ exists since $Y$ is acyclic, and any two choices of $\mathrm{w}$ are $\mathrm{T}$-homotopic.

With these notations, we have the following definition.

Definition 4.1. Let $(K, \theta) \in \zeta(p, n)$. We say that $(K, \theta)$ is an Adem

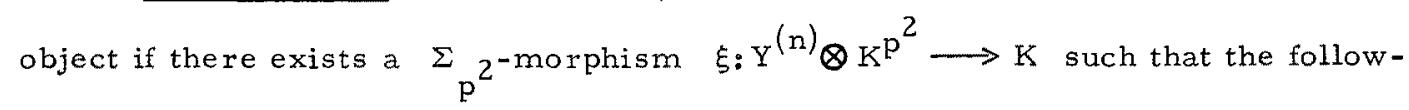
ing diagram is $\mathrm{T}$-homotopy commutative: 


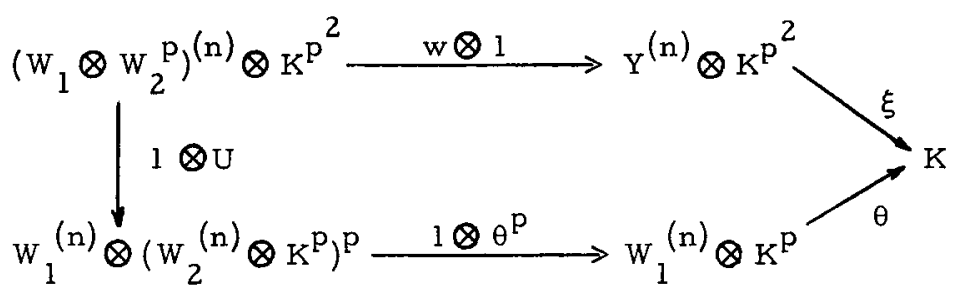

Here $U$ is the evident shuffle map, and is clearly a $T$-morphism $\left(\Sigma p_{p^{2}}\right.$ acts trivially on $\mathrm{K}$ and $\alpha_{i}$ acts trivially on $\left.\mathrm{w}_{1}^{(\mathrm{n})} \otimes \mathrm{K}^{\mathrm{P}}\right)$.

For clarity, we only treat the case $n=\infty$ below. The relations obtained will be valid for operations on $\mathrm{H}_{q}(K),(K, \theta)$ an Adem object of $\zeta(p, n)$, provided that $\mathrm{n}$ is sufficiently large relative to $\mathrm{q}$.

We first show that the tensor product of Adem objects is an Adem object and then use this fact to show that any relations valid on $\mathrm{H}_{q_{i}}(K)$ for all Adem objects $(K, \theta)$ and suitable $q_{i}$ will necessarily be valid on $H_{q}(K)$ for arbitrary q.

Lemma 4.2. If $(K, \theta)$ and $\left(L, \theta^{\prime}\right)$ are Adem objects of $\zeta(p, \infty)$, then $(K \otimes L, \tilde{\theta})$ is an Adem object of $\zeta(p, \infty)$.

Proof. $\tilde{\theta}$ is as defined in Definitions 2.1. By hypothesis, we are given $\xi$ and $\xi^{\prime}$ such that $(K, \xi)$ and $\left(K, \eta^{\prime}\right)$ are objects of $\left.\xi_{\mathrm{p}}{ }^{2}, \infty, z_{\mathrm{p}}\right)$, hence we may define $\tilde{\xi}$ as in Definitions 2.1 so that $\left.(K \otimes L, \tilde{\xi}) \in \zeta_{\mathrm{p}}^{2}, \infty, \mathrm{Z}_{\mathrm{p}}\right)$. We must show that the diagram of Definition 4.1 , for $K \otimes L$, is $T$-homotopy commutative, and this follows easily from a simple chase of a large diagram and the definition of $\tilde{\theta}$ and $\widetilde{\xi}$. The crucial point is the observation that since $W_{1} \otimes W_{2}^{p}$ is $T-f r e e$ and $\mathrm{Y} \otimes \mathrm{Y}$ is acyclic, the following diagram is $\tau$-homotopy commutative where $V$ is the evident shuffle and $\psi: Y \rightarrow Y \otimes Y$ is any given $\Sigma p_{p^{-c o p r o d u c t: ~}}$ 


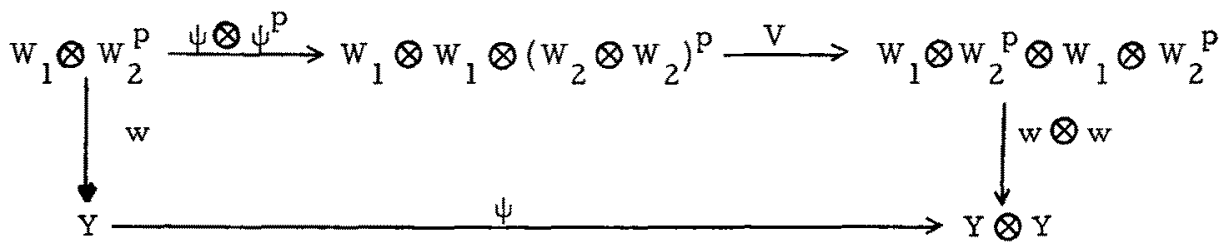

Let $F_{p}$ denote the free associative algebra generated by $\left\{P_{s} \mid s \in Z\right\}$ and, if $p>2,\left\{\beta P_{s} \mid s \in Z\right\}$. Let $J_{p} \subset F_{p}$ denote the two-sided ideal consisting of all elements $a \in F_{p}$ such that $a x=0$ for all $x \in H(K)$ and all Adem objects $(K, \theta) \in \zeta(p, \infty)$. Let $B_{p}=F_{p} / J_{p^{*}} B_{p}$ is a universal Steenrod algebra. Both the classical Steenrod algebra and the Dyer-Lashof algebra [17] are quotients of $\mathrm{B}_{\mathrm{p}}$.

Lemma 4.3. Let a $\in F_{p}$. Let $\left\{q_{i} \mid i \geq 0\right\}$ be a strictly decreasing sequence of integers. Suppose that $a x=0$ for all $x \in \mathrm{H}_{q_{i}}(K), i \geq 0$, and all Adem objects $(K, \theta) \in \zeta(p, \infty)$. Then $a \in J_{p}$.

Proof. Let $\mathrm{K}$ be an Adem object in $\zeta(\mathrm{p}, \infty)$ and let $x \in \mathrm{H}_{\mathrm{q}}(\mathrm{K})$. We must prove that $a x=0$. Choose $r<0$ such that $q+r=q_{i}$ for some $i$. There exists an Adem object $\left(L_{r}, \theta_{r}\right) \in \zeta(p, \infty)$ and a class y $\in H_{r}\left(L_{r}\right)$ such that $P_{0}(y)=y, P_{s}(y)=0$ for $s \neq 0$, and $\beta P_{s}(y)=0$ for all $s$. Such an object can easily be constructed explicitly, but it is quicker to appeal to the results of section 8, which show that the singular cochains of a $(-\mathbf{r})$-sphere, graded by non-positive subscripts, provide such an object. Now $\left(K \otimes L_{r}, \ddot{\theta}\right)$ is an Adem object of $\zeta(p, \infty)$ by the previous lemma. By the external Cartan formula, Corollary 2.7, $a(x \otimes y)=a x \otimes y$. Since $x \otimes y \in H_{q_{i}}\left(K \otimes L_{r}\right), a(x \otimes y)=0$ and therefore $a x=0$, as was to be shown.

The Adem relations will be proven by chosing the diagram of Definition 4.1, and we shall need some information about the homology of $\sigma, \tau$, and $\Sigma_{p^{2}}$. Let $\phi: w_{1} \otimes w_{2} \rightarrow w_{1} \otimes w_{2}^{p}$ be a $\sigma$-morphism over $z_{p}$. Define $\gamma \in \Sigma_{p^{2}}$ by $\gamma(i, j)=(j, i)$. Observe that $\gamma^{2}=1$ and $\gamma \alpha=\beta \gamma$. For $q \in Z$, conjugation by $\gamma$ 
gives a commutative diagram

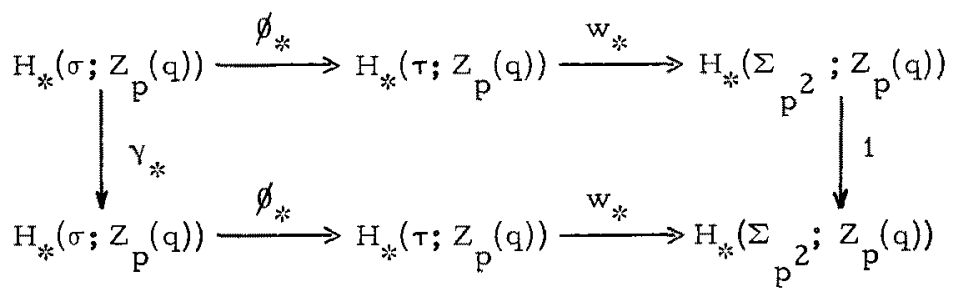

Thus $\mathrm{w}_{*}\left(\emptyset_{*}-\phi_{*} \gamma_{*}\right)=0$. The following lemmas compute $\gamma_{*}$ and $\phi_{*}$. Note that $\mathrm{H}_{*}\left(\tau ; Z_{p}(q)\right)=H_{*}\left(\tau ; Z_{p}\right)$ since $\tau$ contains only even permutations.

Lemma 4.4. $\gamma_{*}$ is given on $H_{* k}\left(\sigma ; z_{p}(q)\right)$ by $\gamma_{* k}\left(e_{i} \otimes e_{j}\right)=(-1)^{i j+m q} e_{j} \otimes e_{i}$. Proof. Define $\gamma_{\#}: w_{1} \otimes w_{2} \rightarrow w_{1} \otimes w_{2}$ by the formula

$$
\gamma_{\#}\left(\alpha^{k} e_{i} \otimes \beta^{l} e_{j}\right)=(-1)^{i j} \alpha^{l} e_{j} \otimes \beta^{k} e_{i} \text {. }
$$

Then $d \gamma_{\#}=\gamma_{\#} d$ and $\gamma_{\#}(\mu x)=\left(\gamma \mu \gamma^{-1}\right) \gamma_{\#}(x)$ for $\mu \in \sigma$ and $x \in W_{1} \otimes W_{2}$. Thus $\gamma_{\#} \otimes \gamma:\left(w_{1} \otimes w_{2}\right) \otimes_{\sigma} z_{p}(q) \rightarrow\left(w_{1} \otimes w_{2}\right) \otimes_{\sigma} z_{p}(q)$ induces $\gamma_{\mu_{*}}$. Since the sign of $\gamma$ is $(-1)^{\mathrm{m}}, \gamma \cdot 1=(-1)^{\mathrm{mq}}$ in $\mathrm{z}_{\mathrm{p}}(\mathrm{q})$, and the result follows.

Before computing $\emptyset_{*}$, we fix notations concerning binomial coefficients.

Notations 4.5. Let $i$ and $j$ be integers. Define $(i, j)=(i+j) ! / i ! j !$ if $i \geq 0$ and $j \geq 0 \quad(0:=1)$ and define $(i, j)=0$ if $i<0$ or $j<0$. Recall that if $i \geq 0$ and $j \geq 0$ have p-adic expansions $i=\sum a_{k} p^{k}$ and $j=\sum b_{k} p^{k}$, then $(i, j) \equiv \prod_{k}\left(a_{k}, b_{k}\right) \bmod p$. Clearly $\left(a_{k}, b_{k}\right) \not \equiv 0 \bmod p$ if and only if $a_{k}+b_{k}<p$ hence $(i, j) \not \equiv 0 \bmod p$ if and only if $\sum\left(a_{k}+b_{k}\right) p$ is the $p$-adic expansion of $i+j$.

Lemma 4.6. $\mathrm{H}_{*}\left(\tau ; \mathrm{Z}_{\mathrm{p}}\right)=\mathrm{H}_{*}\left(\pi ; \mathrm{H}_{*}\left(\nu ; \mathrm{Z}_{\mathrm{p}}\right)^{\mathrm{p}}\right)$ and $\phi_{*}: \mathrm{H}_{*}\left(\sigma ; \mathrm{Z}_{\mathrm{p}}\right) \rightarrow \mathrm{H}_{*}\left(\tau ; \mathrm{Z}_{\mathrm{p}}\right)$ is given by the following formulas (with sums taken over the integers).

(i) If $p=2, \phi_{*}\left(e_{r} \otimes e_{s}\right)=\sum_{k}(k, s-2 k) e_{r+2 k-s} \otimes e_{s-k}^{2} ;$ and

(ii) If $\mathrm{p}>2, \phi_{*}\left(\mathrm{e}_{\mathrm{r}} \otimes \mathrm{e}_{\mathrm{s}}\right)=\sum_{\mathrm{k}}(-1)^{\mathrm{k}} v(\mathrm{~s})(\mathrm{k},[\mathrm{s} / 2]-\mathrm{pk}) \mathrm{e}_{\mathrm{r}+(2 \mathrm{pk}-\mathrm{s})(\mathrm{p}-1)} \& \mathrm{e}_{\mathrm{s}-2 \mathrm{k}(\mathrm{p}-1)}^{\mathrm{p}}$ $-\delta(r) \delta(s-1) \sum_{k}(-1)^{k}(s-1)(k,[s-1 / 2]-p k) e_{r+p+(2 p k-s)(p-1)}^{\otimes e_{s-2 k(p-1)-1}^{p}}$ 
where $\nu(2 j+\varepsilon)=(-1)^{j}(\mathrm{~m} !)^{\boldsymbol{\varepsilon}}$ and $\delta(2 j+\varepsilon)=\varepsilon, j$ any integer, $\varepsilon=0$ or 1 .

Proof. Let $\bar{W}_{2}=W_{2} \otimes_{v} z_{p}=H_{*}\left(v ; z_{p}\right)$. By the definition of the action of $\tau$ on $W_{1} \otimes W_{2}^{p}$, we have that $\left(W_{1} \otimes w_{2}^{p}\right) \otimes_{\tau} z_{p}=W_{1} \otimes_{\pi} \bar{W}_{2}^{p}$ as a $z_{p}$-complex, and the first part follows. Of course, $H_{*}\left(\tau ; Z_{p}\right)$ is now computed by Lemma 1.3 . $\emptyset_{*}$ could be computed directly, but it is simpler to use topology. Let $K\left(Z_{p}, 1\right)=E / \nu$, where $\nu$ operates properly on the acyclic space $E$, so that, by $\left[14\right.$, IV 11], $\quad C_{*}(E)=Z_{p} v \otimes C_{*}(E / \nu)$, with $Z_{p}$ coefficients. Let $D: E \rightarrow E^{p}$ be the iterated diagonal. Then $1 \otimes D: W_{1} \otimes C_{*}(E) \rightarrow W_{1} \otimes C_{*}\left(E^{\mathrm{P}}\right)$ is a $\sigma-m o r p h i s m$, and we shall obtain a $\sigma$-morphism $\Phi: W_{1} \otimes C_{*}\left(E^{P}\right) \rightarrow W_{1} \otimes C_{*}(E)^{p}$ in Lemma 7.1. Let $\mathrm{d}=\Phi(\mathrm{l} \otimes D)$ and let $\mathrm{f}: \mathrm{W}_{2} \rightarrow \mathrm{C}_{*}(E)$ be a $v$-morphism over $\mathrm{Z}_{\mathrm{p}}$. Since $W_{1} \otimes W_{2}$ is $\sigma-f$ ree and $W_{1} \otimes C_{*}(E)^{P}$ is acyclic (and $\tau$-free, with the evident $\tau$-action), the following diagram is $\sigma$-homotopy commutative.

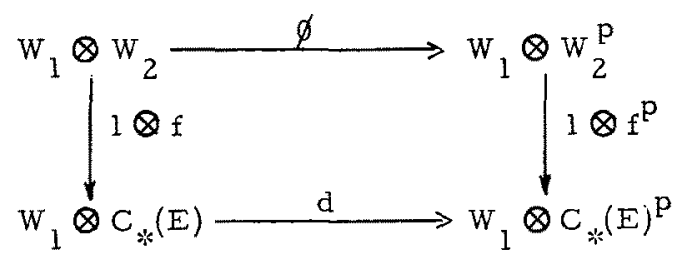

Therefore $\phi_{*}=d_{*}: H_{*}\left(\sigma ; Z_{p}\right) \rightarrow H_{* *}\left(\tau ; Z_{p}\right)$, and this can clearly be computed from the quotient map $\mathrm{d}: \mathrm{W}_{1} \otimes_{\pi} \mathrm{C}_{*}(\mathrm{E} / v) \rightarrow \mathrm{W}_{1} \otimes_{\pi} \mathrm{C}_{*}(\mathrm{E} / v)^{\mathrm{P}}$. We shall prove the following formulas in Proposition 9.1.

(a) If $\mathrm{p}=2, \mathrm{~d}_{*}\left(\mathrm{e}_{\mathrm{r}} \otimes \mathrm{e}_{\mathrm{s}}\right)=\sum_{\mathrm{k}} \mathrm{e}_{\mathrm{r}+2 \mathrm{k}-\mathrm{s}} \otimes \mathrm{P}_{* k}{ }^{\mathrm{k}}\left(\mathrm{e}_{\mathrm{s}}\right)^{2} ;$ and

(b) If $\mathrm{p}>2, \quad \mathrm{~d}_{*}\left(\mathrm{e}_{\mathrm{r}} \otimes \mathrm{e}_{\mathrm{s}}\right)=\sum_{\mathrm{k}}(-1)^{\mathrm{k}} v(\mathrm{~s}) \mathrm{e}_{\mathrm{r}+(2 \mathrm{pk}-\mathrm{s})(\mathrm{p}-1)} \otimes \mathrm{P}_{*}{ }^{\mathrm{k}}\left(\mathrm{e}_{\mathrm{s}}\right)^{\mathrm{p}}$

$$
-\delta(r) \sum_{k}(-1)^{k} v(s-1) e_{r+p+(2 p k-s)(p-1)} \otimes P_{*}^{k} \beta\left(e_{s}\right)^{p} .
$$

Here the $P_{*}^{k}$ are the duals to the Steenrod operations in $H^{*}\left(K\left(Z_{p}, 1\right) ; Z_{p}\right)=$ $\mathrm{H}^{*}\left(\nu ; \mathrm{Z}_{\mathrm{p}}\right)$. The latter operations satisfy $\mathrm{P}^{\circ}=1$ and the internal Cartan formula, hence, by (1.2), if $w_{t}$ is dual to $e_{t}$, then

(c) If $\mathrm{p}=2, \mathrm{P}^{\mathrm{k}}\left(\mathrm{w}_{\mathrm{t}}\right)=(\mathrm{k}, \mathrm{t}-\mathrm{k}) \mathrm{w}_{\mathrm{k}+\mathrm{t}}$, hence $\mathrm{P}_{*}^{\mathrm{k}}\left(\mathrm{e}_{\mathrm{s}}\right)=(\mathrm{k}, \mathrm{s}-2 \mathrm{k}) \mathrm{e}_{\mathrm{s}-\mathrm{k}}$; and 
(d) If $p>2, \quad \mathrm{P}^{\mathrm{k}}\left(\mathrm{w}_{\mathrm{t}}\right)=(\mathrm{k},[\mathrm{t} / 2]-\mathrm{k}) \mathrm{w}_{\mathrm{t}+2 \mathrm{k}(\mathrm{p}-1)}$, hence

$$
P_{*}^{k}\left(e_{s}\right)=(k,[s / 2]-p k) e_{s-2 k(p-1)} \text {. }
$$

Combining (a) and (c), we obtain (i). Combining (b), (d), and $\beta\left(e_{i}\right)=\delta(i-1) e_{i-1}$, we obtain (ii).

Theorem 4.7. The following relations among the $\mathrm{P}_{\mathrm{s}}$ and $\beta P_{\mathrm{s}}$ are valid on all homology classes of all Adem objects in $\zeta(p, \infty)$.

(ii) If $p>2$ and $a>p b, P_{a} P_{b}=\sum_{i}(-1)^{a+i}(p i-a, a-(p-1) b-i-1) P_{a+b-i} P_{i}$ and $\beta P_{a} P_{b}=\sum_{i}(-1)^{a+i}(p i-a, a-(p-1) b-i-1) \beta P_{a+b-i} P_{i}$

$$
\text { If } \begin{aligned}
p>2 \text { and } a \geq p b & , P_{a} \beta P_{b}=\sum_{i}(-1)^{a+i}(p i-a, a-(p-1) b-i) \beta P_{a+b-i} P_{i} \\
& -\sum_{i}(-1)^{a+i}(p i-a-1, a-(p-1) b-i) P_{a+b-i} \beta P_{i}
\end{aligned}
$$

and

$$
\beta P_{a} \beta P_{b}=-\sum_{i}(-1)^{a+i}(p i-a-1, a-(p-1) b-i) \beta P_{a+b-i} \beta P_{i}
$$

Proof. Note first that the second relations of (ii) and (iii) are implied by the first for objects which are reduced mod p, but a re logically independent in our general setting. Let $(K, \theta)$ be an Adem object in $\zeta(p, \infty)$ and let $x \in H_{q}(K)$. Definition 4.1 implies that we have a $\mathrm{z}_{\mathrm{p}}$-homotopy commutative diagram

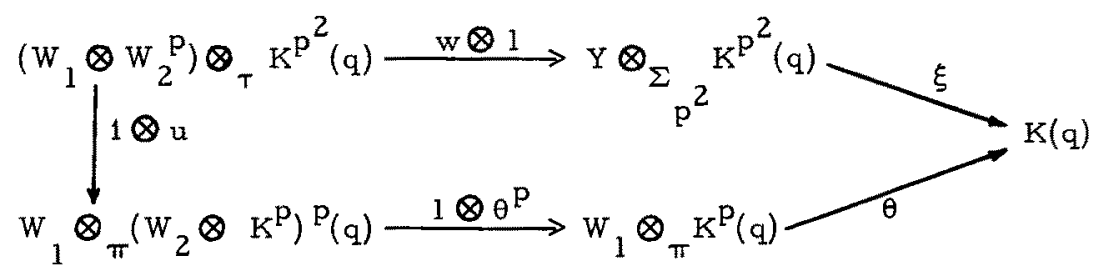

Since $x^{p^{2}}$ is $\Sigma_{p^{2}}$ invariant in $K^{p^{2}}(q)$, we have, for all $r$ and $s$,

(a) $\quad \xi_{*}(w \otimes 1)_{*}\left(e_{r} \otimes e_{s}^{p} \otimes x^{p^{2}}\right)=\xi_{*}\left(w_{*}\left(e_{r} \otimes e_{s}^{p}\right) \otimes x^{p^{2}}\right)$. 
In the other direction, $U$ introduces the $\operatorname{sign}(-1)^{\mathrm{mq} s}$ and we have

(b) $\quad \theta_{*}\left(1 \otimes \theta^{p}\right)_{*}(1 \otimes U)_{*}\left(e_{r} \otimes e_{S}^{p} \otimes x^{p^{2}}\right)=(-1)^{m q s} D_{r} D_{s}(x)$.

Since $\mathrm{w}_{*} \oint_{*}=\mathrm{w}_{*} \oint_{*^{*}} \gamma_{*}$, Lemma 4.4 gives the formula

(c) $\quad \mathrm{w}_{*} \phi_{*}\left(\mathrm{e}_{\mathrm{r}} \otimes \mathrm{e}_{\mathrm{s}}\right)=(-1)^{\mathrm{rs}+\mathrm{mq}} \mathrm{w}_{* *} \phi_{*}\left(\mathrm{e}_{\mathrm{s}} \otimes \mathrm{e}_{\mathrm{r}}\right)$.

Combining formulas (a) and (c), we obtain the formula

(d) $\quad \xi_{* k}(w \otimes 1)_{* k}\left(\emptyset_{* k}\left(e_{r} \otimes e_{s}\right) \otimes x^{p^{2}}\right)=(-1)^{r s+m q} \xi_{*}(w \otimes 1)_{*}\left(\emptyset_{*}\left(e_{s} \otimes e_{r}\right) \otimes x^{p^{2}}\right)$.

In view of (b), (d) gives relations on iterated operations, and these relations are explicit since $\phi_{*}$ is known. We prove the three parts of the theorem successively In all parts, the statements about binomial coefficients are verified by writing out the p-adic expansions of the relevant integers and appealing to the remarks in Notations 4.5.

(i) By (b) and (d), Lemma 4.6 implies the formula

(e) $\quad \sum_{k}(k, s-2 k) D_{r+2 k-s} D_{s-k}(x)=\sum_{\ell}(\ell, r-2 \ell) D_{s+2 \ell-r} D_{r-\ell}(x)$.

Formula (e) is valid for all $r$ and $s$, and we set $r=a-2 q$ and $s=b-q$ for our fixed $a>2 b$. If we then change variables to $j=b-k$ and $i=a-q-\ell$ and apply Definition 2. 2, we obtain

(f) $\quad \sum_{j}(b-j, 2 j-b-q) P_{a+b-j} P_{j}(x)=\sum_{i}(a-q-i, 2 i-a) P_{a+b-i} P_{i}(x)$.

The condition $a>2 b$ guarantees that the same terms do not appear with non-zero coefficients on both sides of (f). Now suppose that $q=b-2^{t}+1$ for some $t>0$.

Then, if $\mathbf{j} \neq \mathrm{b}$,

$$
(b-j, 2 j-b-q)=\left(b-j, 2^{t}-1-2(b-j)\right)=0 .
$$

On the right side of $(f), P_{a+b-i} P_{i}(x)=0$ unless $2^{t}-1=b-q \geq 2 i-a$, while if $2^{t}>2 i-a$, then

$$
(a-q-i, 2 i-a)=\left(2 i-a, a-b-i-1+2^{t}\right)=(2 i-a, a-b-i-1)
$$

Thus (f) reduces to the desired relation (i) when $q=b-2^{t}+1$ for some $t>0$. By Lemma 4. 3, it follows that (i) holds for all q. 
(ii) Observe that $\beta w_{*}=w_{*} \beta$ and that, by the proof of part (v) of Proposition 2. 3, the Bockstein operation $\beta$ in $H_{*}\left(\tau ; Z_{p}\right)$ is given by $\beta\left(e_{k} \otimes e_{l}^{p}\right)=\beta\left(e_{k}\right) \otimes e_{l}^{p}$, where $\beta\left(e_{k}\right)=\delta(k-1) e_{k-1}$. Now since $(c)$ holds with $\phi_{*}$ replaced by $\beta \phi_{*}$, so does (d); that is,

(d') $\quad \xi_{*}(w \otimes 1)_{* *}\left(\beta \phi_{* *}\left(e_{r} \otimes e_{s}\right) \otimes x^{p^{2}}\right)=(-1)^{r s+m q} \xi_{*}(w \otimes 1)_{* *}\left(\beta \phi_{* k}\left(e_{s} \otimes e_{r}\right) \otimes x^{p^{2}}\right)$.

Replace $r$ and $s$ by $2 r$ and $2 s$ in (d) and ( $\left.d^{\prime}\right)$ and let $\varepsilon=0$ or 1 ; then, by (b) and Lemma 4.6, (d) and (d') imply the following formula for $\varepsilon=0$ and $\varepsilon=1$, respectively.

(g)

$$
\begin{aligned}
& \sum_{k}(-1)^{k} \nu(2 s)(k, s-p k) D_{2 r+(2 p k-2 s)(p-1)-\varepsilon} D_{2 s-2 k(p-1)}(x) \\
& \quad=\sum_{\ell}(-1)^{\ell+m q} v(2 r)(\ell, r-p \ell) D_{2 s+(2 p \ell-2 r)(p-1)-\varepsilon} D_{2 r-2 \ell(p-1)}(x) .
\end{aligned}
$$

In $(g)$, set $r=a(p-1)-p q m$ and $s=b(p-1)-q m$ and change variables to $j=b-k$ and $i=a-m q-\ell$. Let $\beta^{\circ} P_{s}=P_{s}$ and $\beta^{1} P_{s}=\beta P_{s}$, by abuse; then, by Definitions 2. 2 and a check of constants, we obtain

$$
\begin{aligned}
& \sum_{j}(-1)^{b+j}(b-j, p j-b-m q) \beta^{\varepsilon} P_{a+b-j} P_{j}(x) \\
& \quad=\sum_{i}(-1)^{a+i}(a-m q-i, p i-a) \beta^{\varepsilon} P_{a+b-i} P_{i}(x) .
\end{aligned}
$$

Again, $a>p b$ ensures that the same terms do not appear on both sides of $(h)$. Now suppose that $q=2 b-2\left(1+p+\ldots+p^{t-1}\right), t>0$. Then $(b-j, p j-b-m q)=0$ unless $j=b$ and, on the other side of $(h), \beta^{E} P_{a+b-i} P_{i}(x)=0$ unless $1+\ldots+p^{t-1}=b-q / 2 \geq p i-a$, when $p^{t}>p i-a$ implies

$$
(a-m q-i, p i-a)=\left(p i-a, a-(p-1) b+p^{t}-1-i\right)=(p i-a, a-(p-1) b-i-1) .
$$

Thus (h) reduces to the desired relations (ii) when $q=2 b-2\left(1+\ldots+p^{t-1}\right)$ for some $t>0$. By Lemma 4.3, it follows that (ii) holds for all $q$.

(iii) Replace $x$ and $s$ by $2 r$ and $2 s-1$ in (d) and (d'); then, by (b) and Lemma 4.6, (d) and ( $\left.d^{\prime}\right)$ imply the following formula for $\varepsilon=0$ and $\varepsilon=1$, respectively. 
(i) $\quad \sum_{k}(-1)^{k+m q} v(2 s-1)(k, s-1-p k) D_{2 r+(2 p k-2 s+1)(p-1)-\varepsilon} D_{2 s-1-2 k(p-1)}{ }^{(x)}$

$$
\begin{aligned}
& =(1-\varepsilon) \sum_{\ell}(-1)^{\ell+m_{v}(2 r)(\ell, r-p \ell) D_{2 s-1+(2 p \ell-2 r)(p-1)} D_{2 r-2 \ell(p-1)}(x)} \\
& -\sum_{\ell}(-1)^{\ell} v(2 r-1)(\ell, r-1-p \ell) D_{2 s+(2 p \ell-2 r+1)(p-1)-\varepsilon} D_{2 r-1-2 \ell(p-1)}(x) .
\end{aligned}
$$

In (i), set $r=a(p-1)-p q m$ and $s=b(p-1)-q m$ and change variables to $j=b-k$ and $i=a-m q-\ell$. By Definitions 2.2, we obtain

$$
\text { (j) } \begin{aligned}
& \sum_{j}(-1)^{b+j}(b-j, p j-b-m q-1) \beta^{\varepsilon} P_{a+b-j} \beta P_{j}(x) \\
&=(1-\varepsilon) \sum_{i}(-1)^{a+i}(a-m q-i, p i-a) \beta P_{a+b-i} P_{i}(x) \\
&-\sum_{i}(-1)^{a+i}(a-m q-i, p i-a-1) \beta^{\varepsilon} P_{a+b-i} \beta P_{i}(x) .
\end{aligned}
$$

Again, $a \geq p b$ ensures that the same terms do not occur on both sides of the

equation. Now suppose that $q=2 b-2 p^{t}, t>0$. Then $(b-j, p j-b-m q-1)=0$ unless $j=b$. On the other side of $(j), \quad \beta P_{a+b-i} P_{j}(x)=0$ unless $p^{t}>$ pi-a, when

$$
(a-m q-i, p i-a)=\left(p i-a, a-(p-1) b-i+(p-1) p^{t}\right)=(p i-a, a-(p-1) b-i),
$$

and $\beta^{\varepsilon} P_{a+b-i} \beta P_{i}(x)=0$ unless $p^{t}>$ pi-a-1, when

$$
(a-m q-i, p i-a-1)=\left(p i-a-1, a-(p-1) b-i+(p-1) p^{t}\right)=(p i-a-1, a-(p-1) b-i) \text {. }
$$

Thus (j) reduces to the desired relations (iii) when $q=2 b-2 p^{t}$ for some $t<0$, and Lemma 4.3 implies that (iii) holds for all q.

Remark 4.8. It should be observed, for use in section 9, that the relations (f), $(h)$, and $(j)$ derived in the proof above are valid for arbitrary integers $a$ and $b$ (without the restrictions $\mathrm{a}>\mathrm{pb}$ or $\mathrm{a} \geq \mathrm{pb}$ ). Indeed, these conditions on $\mathrm{a}$ and $b$ were only required in order to obtain disjoint non-trivial terms on the two sides of the cited equations. 


\section{Reindexing for cohomology.}

We have geared our discussion to homology, but the reformulation appropriate to cohomology is obtained by a mino $r$ and standard change of notation. Thus let $\mathrm{K}$ be a $\mathrm{Z}_{\mathrm{p}}$-complex $\mathrm{Z}$-graded by superscripts, with $\mathrm{d}$ of degree plus one. If we regrade $K$ by $K_{-q}=K^{q}$, then the theory of the previous sections applies. Equivalently, we can regrade $W$ by non-positive superscripts and reformulate the theory. Obviously, this in no way changes the proofs. Let $(K, \theta) \in \zeta(p, \infty)$, with $K$ and $W$ graded by superscripts, and let $x \in H^{q}(K)$. Then $D_{i}(x)=\theta_{*}\left(e^{-i} \otimes x^{p}\right) \in H^{p q-i}(K), i \geq 0$, and we may define $P^{s}(x)=P_{-s}(x)$ and, if $p>2, \beta P^{s}(x)=\beta P_{-s}(x)$. Explicitly, $P^{s}(x)$ and $\beta P^{s}(x)$ are defined by the formulas

(1) If $p=2, P^{s}(x)=D_{q-s}(x) \in H^{q+s}(K)$, where $D_{i}=0$ for $i<0$; and

(2) If $p>2, \mathrm{P}^{s}(\mathrm{x})=(-1)^{\mathrm{s}} v(-\mathrm{q}) \mathrm{D}(\mathrm{q}-2 \mathrm{~s})(\mathrm{p}-1)^{(\mathrm{x}) \in \mathrm{H}^{\mathrm{q}+2 \mathrm{~s}(\mathrm{p}-1)}(\mathrm{K}) \text { and }}$ $\beta P^{s}(x)=(-1)^{s} \nu(-q) D(q-2 s)(p-1)-1(x) \in H^{q+2 s(p-1)+1}(K)$, where
$D_{i}=0$ for $i<0$ and if $q=2 j-\varepsilon, \varepsilon=0$ or 1 , then $v(-q)=(-1)^{j}(m !)^{\varepsilon}$.

Of course, if $\mathrm{p}=2$, we should write $\mathrm{P}^{\mathrm{s}}=\mathrm{Sq}^{\mathrm{s}}$ in order to conform to standard notations, but we prefer to retain the notation $\mathrm{P}^{\mathrm{s}}$. In this way, the Cartan formula and Adem relations are formally the same in the cases $p=2$ and $\mathrm{p}>2$.

The $P^{S}$ and $\beta P^{S}$ are natural homomorphisms and a re defined for all integers s. If $(K, \theta) \in C(p, \infty)$ and $x \in H^{q}(K)$, then

(3) If $p=2, P^{s}(x)=0$ if $s>q$ and $p^{q}(x)=x^{2}$; and

(4) If $p>2, P^{s}(x)=0$ if $2 s>q, \beta P^{s}(x)=0$ if $2 s \geq q$, and $P^{s}(x)=x^{p}$ if $2 s=q$. Note that we do not claim that $P^{5}(x)=0$ if $s<0$ or that $P^{O}=1$; these formulas are not true in general. If $(K, \theta)$ is unital, then $P^{s}(e)=0$ for $s \neq 0$. If $(K, \theta)$ is reduced $\bmod p$, then 
(5) $\beta P^{s-1}=s P^{s}$ if $p=2$ and $\beta P^{s}$ is the composition of $P^{s}$ and the Bockstein $\beta$ if $p>2$.

The external Cartan formula now reads

$$
\begin{aligned}
& P^{s}(x \otimes y)=\sum_{i+j=s} P^{i}(x) \otimes P^{j}(y) \text { and, if } p>2, \\
& \beta P^{s+1}(x \otimes y)=\sum_{i+j=s}\left(\beta P^{i+1}(x) \otimes P^{j}(y)+(-1)^{\operatorname{deg} x} P^{i}(x) \otimes \beta P^{j+1}(y)\right) .
\end{aligned}
$$

We have $\sigma P^{\mathbf{S}}=\mathrm{P}_{\sigma}^{\mathbf{s}}$ and $\sigma \beta \mathrm{P}^{\mathbf{S}}=-\beta \mathrm{P}_{\sigma}^{\mathbf{s}}$ and of course the Kudo transgression theorem takes on a more familiar form with grading by superscripts in case (ii). The Adem relations, reformulated in terms of the $P^{S}$, take on the form given in the following corollary.

Corollary 5.1. The following relations among the $\mathrm{P}^{\mathrm{s}}$ and $\beta \mathrm{P}^{\mathrm{s}}$ are valid on all cohomology classes of all Adem objects in $6(p, \infty)$

(i) If $\mathrm{p} \geq 2, \mathrm{a}<\mathrm{pb}$, and $\varepsilon=0$ or 1 if $\mathrm{p}>2, \varepsilon=0$ if $\mathrm{p}=2$, then

$$
\beta^{\varepsilon} P^{a} P^{b}=\sum_{i}(-1)^{a+i}(a-p i,(p-1) b-a+i-1) \beta^{\varepsilon} P^{a+b-i} P^{i}
$$

(ii) If $\mathrm{p}>2, \mathrm{a} \leq \mathrm{pb}$, and $\boldsymbol{\varepsilon}=0$ or 1 , then

$$
\begin{aligned}
\beta^{\varepsilon} P^{a} \beta P^{b} & =(1-\varepsilon) \sum_{i}(-1)^{a+i}(a-p i,(p-1) b-a+i-1) \beta P^{a+b-i} P^{i} \\
& -\sum_{1}(-1)^{a+i}(a-p i-1,(p-1) b-a+i) \beta^{\varepsilon} P^{a+b-i} \beta P^{i}
\end{aligned}
$$

(where, by abuse of notation, $\beta^{\circ} P^{s}=P^{s}$ and $\beta^{l} P^{s}=\beta P^{s}$ ).

While the two forms of the Adem relations given in Theorem 4.7 and the corollary are completely equivalent, they work out quite differently in practice. The relations of Theorem 4.7 apply to positive complexes, in homology, with $\mathrm{a}, \mathrm{b} \geq 0$; but $\mathrm{a}, \mathrm{b} \geq 0$ in Theorem 4.7 corresponds to $\mathrm{a}, \mathrm{b} \leq 0$ in the corollary, which is designed for use in cohomology with $a, b \geq 0$. For this reason, the DyerLashof algebra [ 17], which operates on the homology of infinite loop spaces, is a very different algebraic object than the classical Steenrod algebra. 


\section{Cup-i products, Browder operations, and higher Bocksteins.}

We here discuss $v_{i}$-products and certain homology operations of two variables, which were first studied by Browder in [4]; these operations occur in the presence of a $u_{n}$-product and the absence of a $u_{n+1}$-product and are central to the study of the homology of $(n+1)$-fold loop spaces. We shall also obtain a very useful result, Proposition 6.8, on higher Bockstein operations. In section 10 , we shall show that this result suffices to give a complete computation of the mod $p$ cohomology Bockstein spectral sequence of $K(\pi, n)$ for any Abelian group $\pi$ and any prime $p$.

Throughout this section, $\Lambda$ is a commutative ring, $\pi$ is the cyclic group of order 2 with generator $\alpha$, and $W$ is the canonical $\Lambda \pi$-free resolution of $\Lambda$. Let $\Delta_{i}=\alpha+(-1)^{i} \in \Lambda \pi$, so that $d\left(e_{i}\right)=\Delta_{i} e_{i-1}$ for $i \geq 1$. If $(K, \theta) \in \zeta(\pi, n, \Lambda)$, then we may assume that the restriction of $\theta$ to $e_{0} \otimes K \otimes K$ agrees with the given product on $\mathrm{K}$ by (i) of Definition 2. 1 .

Definition 6.1. Let $(K, \theta) \in \zeta(\pi, n, \Lambda)$ and let $x \in K_{q}$ and $y \in K_{r}$. For $0 \leq i \leq n$, define $x \cup_{i} y=(-1)^{\frac{1}{2} i(i+1)} \theta\left(e_{i} \otimes x \otimes y\right)$. Then $U_{0}$ is the product on $K$ and if $i>0$, then $v_{i}: K \otimes K \rightarrow K$ is a chain homotopy of degree $i$ from $v_{i-1}$ to $(-1)^{i-1} v_{i-1} \cdot \alpha$; that is,

(i) $\quad d\left(x v_{i} y\right)=(-1)^{i} d(x) v_{i} y+(-1)^{i+q} x v_{i} d(y)+x v_{i-1} y+(-1)^{i+q r} y u_{i-1} x$.

If $\Lambda=Z_{2}$ and $x \in K_{q}$ is a cycle, then $P_{i+q}\{x\}=D_{i}\{x\}=\left\{x u_{i} x\right\}$, which, in cohomology, was Steenrod's first definition [25] of the squares. We now define the Browder operations for $(K, \theta) \in \zeta(\pi, n, \Lambda)$.

Definition 6.2. Let $(K, \theta) \in \zeta(\pi, n, \Lambda), n<\infty$, and let $x \in \mathrm{H}_{\mathrm{q}}(K)$ and $y \in H_{r}(K)$. Observe that if $a$ and $b$ are representative cycles for $x$ and $y$, then $\Delta_{n+1} e_{n} \otimes a \otimes b$ is a cycle in $w^{(n)} \otimes K^{2}$ whose homology class $\Delta_{n+1} e_{n} \otimes x \otimes y$ depends only on $x$ and $y$. Define $\lambda_{n}(x, y) \in H_{q+r+n}(K)$ by 
$\lambda_{\mathrm{n}}(\mathrm{x}, \mathrm{y})=(-1)^{\mathrm{nq}+1} \theta_{*}\left(\Delta_{\mathrm{n}+1} e_{\mathrm{n}} \otimes \mathrm{x} \otimes \mathrm{y}\right)$. Note that we have chosen not to pass to equivariant homology; of course, we can do so, and, in $W^{(n)} \otimes_{\pi} K^{2}$,

$$
(-1)^{n q+1} \Delta_{n+1} e_{n} \otimes a b_{b}=(-1)^{n q+n} e_{n} \otimes a \otimes b-(-1)^{n q+q r_{e}} \otimes_{n} \otimes a
$$

Thus $\lambda_{n}(x, y)$ is represented by $(-1)^{n q+n+\frac{1}{2} n(n+1)}\left(a u_{n} b-(-1)^{n+q r_{b}} u_{n} a\right)$.

The following proposition contains many of the elementary properties of the $\lambda_{n}$; its proof is immediate from the definition.

Proposition 6.3. Let $(K, \theta) \in \zeta(\pi, n, \Lambda), n<\infty$, and consider $\lambda_{n}: \mathrm{H}_{\mathrm{q}}(\mathrm{K}) \times \mathrm{H}_{\mathrm{r}}(\mathrm{K}) \longrightarrow \mathrm{H}_{\mathrm{q}+\mathrm{r}+\mathrm{n}}(\mathrm{K})$.

(i) $\lambda_{n}$ induces a homomorphism $\lambda_{n}: H_{q}(K) \otimes H_{r}(K) \rightarrow H_{q+r+n}(K)$

(ii) If $f: K \rightarrow K^{\prime}$ is a morphism in $\zeta(\pi, n, \Lambda)$, then $\lambda_{n}\left(f_{* *} \otimes f_{* k}\right)=f_{* *^{*}} \lambda_{n}$

(iii) If $\theta$ is the restriction to $W^{(n)} \otimes K^{2}$ of $\theta^{\prime}: W^{(n+1)} \otimes K^{2} \rightarrow K$, then $\lambda_{n}=0$

(iv) If $\mathrm{n}=0$, then $\lambda_{0}(\mathrm{x}, \mathrm{y})=\mathrm{xy}-(-1)^{\mathrm{qr}} \mathrm{yx}$

(v) If $(K, \theta)$ is unital and the restriction of $\theta$ to $W^{(n)} \otimes(e \otimes K+K \otimes$ e) is homotopic to $\varepsilon \otimes \varnothing, \emptyset$ the product, then $\lambda_{n}(x, e)=0=\lambda_{n}(e, y)$.

(vi) $\lambda_{n}(x, y)=(-1)^{q r+1+n(q+r+1)} \lambda_{n}(y, x)$ and, if $2=0$ in $\Lambda, \lambda_{n}(x, x)=0$

(Note that the first part implies $2 \lambda_{n}(x, x)=0$ if $n+q$ is even.)

The $\lambda_{n}$ satisfy the following analog of the external Cartan formula.

Proposition 6.4. Let $(K, \theta)$ and $\left(L, \theta^{\prime}\right)$ be in $\zeta(\pi, n, \Lambda), n<\infty$ and $\Lambda$ a field. Let $x \in \mathrm{H}_{q}(K), x^{\prime} \in \mathrm{H}_{\mathbf{r}}(\mathrm{K}), y \in \mathrm{H}_{S}(L)$ and $\mathrm{y}^{\prime} \in \mathrm{H}_{t}(\mathrm{~L})$. Then $\lambda_{n}\left(x \otimes y, x^{\prime} \otimes y^{\prime}\right)=(-1)^{r(s+n)} x x^{\prime} \otimes \lambda_{n}\left(y, y^{\prime}\right)+(-1)^{s(r+t+n)} \lambda_{n}\left(x, x^{\prime}\right) \otimes y^{\prime} y$.

Proof. Let $a, a^{\prime}, b, b^{\prime}$ represent $x, x^{\prime}, y, y^{\prime}$ respectively. Let $c=(-1)^{n(q+s)+1} \tilde{\theta}\left(\Delta_{n+1} e_{n} \otimes a \otimes b \otimes a^{\prime} \otimes b^{\prime}\right)$, so that $c$ represents $\lambda_{n}\left(x \otimes y, x^{\prime} \otimes y^{\prime}\right)$. By (1) of Definition 1.2, 


$$
\begin{aligned}
\psi\left(e_{n}\right)= & \sum_{j=0}^{n} e_{j} \otimes \alpha^{j} e_{n-j} \text { in } w \text {, and the definition of } \tilde{\theta} \text { shows that } \\
c= & \sum_{j=0}^{n}(-1)^{r s+n(1+r+s)-j(q+r)} \theta\left(e_{j} \otimes a \otimes a^{\prime}\right) \otimes \theta^{\prime}\left(\alpha^{j} e_{n-j} \otimes b \otimes b^{\prime}\right) \\
& -\sum_{j=0}^{n}(-1)^{r s+n(r+s)-j(q+r)} \theta\left(\alpha e_{j} \otimes a \otimes a^{\prime}\right) \otimes \theta^{\prime}\left(\alpha^{j+l} e_{n-j} \otimes b \otimes b^{\prime}\right)
\end{aligned}
$$

Let $e=\sum_{j=1}^{n}(-1)^{r s+n(r+s)-(j+1)(q+r)} \theta\left(e_{j} \otimes a \otimes a^{\prime}\right) \otimes \theta^{\prime}\left(\alpha^{j} e_{n+l-j} \otimes b \otimes b^{\prime}\right) . \quad$ Then a straightfo rward calculation demonstrates that

$$
\begin{aligned}
& c+d(e)=(-1)^{r(s+n)+s n+n} \theta\left(e_{0} \otimes a \otimes a^{\prime}\right) \otimes \theta^{\prime}\left(\Delta_{n+1} e_{n} \otimes b \otimes b^{\prime}\right) \\
& +(-1)^{s(r+n)+q n+n} \theta\left(\Delta_{n+1} e_{n} \otimes a \otimes a^{\prime}\right) \otimes \theta^{\prime}\left(\alpha^{n+1} e_{0} \otimes y \otimes y^{\prime}\right) .
\end{aligned}
$$

Since $L$ is homotopy commutative for $n>0, \theta^{\prime}\left(\alpha^{n+1} e_{0} \otimes b \otimes b^{\prime}\right)$ represents $(-1)^{\text {st }} \mathrm{y}^{\prime} \mathrm{y}$ for any $\mathrm{n}$, and the result follows.

We next prove that the $\lambda_{n}$ commute with suspension.

Proposition 6.5. Let $\left(K^{\prime}, \theta^{\prime}\right) \in \zeta(\pi, n+1, \Lambda)$ and $\left(K^{\prime \prime}, \theta^{\prime \prime}\right) \in \zeta(\pi, n, \Lambda)$. Let $\mathrm{K}$ be a $\Lambda$-complex and let $\mathrm{f}: \mathrm{K}^{\prime} \rightarrow \mathrm{K}$ and $\mathrm{g}: \mathrm{K} \rightarrow \mathrm{K}^{\prime \prime}$ be morphisms of complexes such that $g f=0$. Define

$$
\tilde{K}=w^{(n+1)} \otimes f\left(K^{\prime}\right)^{2}+\bar{w}^{(n+1)} \otimes f\left(K^{\prime}\right) \otimes K+w^{(n)} \otimes K^{2},
$$

where $\bar{W}^{(n+1)}=W^{(n)}+\Lambda_{e}{ }_{n+1}\left(\alpha e_{n+1} \notin \bar{W}^{(n+1)}\right)$. Suppose given a $\pi$-morphism $\theta: \widetilde{K} \rightarrow K$ such that the following diagram is commutative:

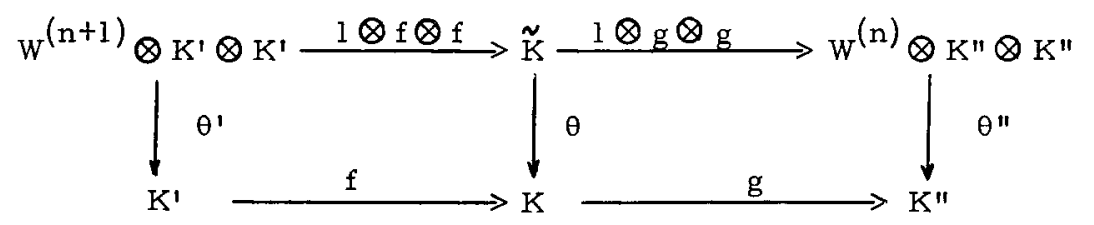

Let $x, y \in \operatorname{Ker} f_{*^{*}}$ Then $\sigma \lambda_{n+1}(x, y)=\lambda_{n}(\sigma x, \sigma y) \in$ Coker $g_{*^{*}}$

Proof. Let $a^{\prime} \in K_{q}^{\prime}$ and $b^{\prime} \in K_{r}^{\prime}$ represent $x$ and $y$ respectively. Let $a=f\left(a^{\prime}\right)$ and $b=f\left(b^{\prime}\right)$ and choose $u \in K_{q+1}$ and $v \in K_{r+1}$ such that $d(u)=a$ 
and $d(v)=b$. Define $c \in \tilde{\mathrm{K}}$ by

$$
c=(-1)^{q}{ }_{n+1} e_{n} \otimes u \otimes v-(-1)^{n+q} e_{n+1} \otimes a \otimes v-(-1)^{r(q+1)} e_{n+1} \otimes b \otimes u .
$$

Then a straightforward calculation demonstrates that

$$
\mathrm{d}(\mathrm{c})=\mathrm{e}_{\mathrm{n}+1} \otimes \mathrm{a} \otimes \mathrm{b}+(-1)^{\mathrm{n}+\mathrm{qr}} \mathrm{e}_{\mathrm{n}+1} \otimes \mathrm{b} \otimes \mathrm{a}=(-1)^{\mathrm{n}} \Delta_{\mathrm{n}+2} \mathrm{e}_{\mathrm{n}+1} \otimes \mathrm{a} \otimes \mathrm{b}
$$

Thus $(-1)^{(n+1) q+1} f \theta^{\prime}\left(\Delta_{n+2} e_{n+1} \otimes a^{\prime} \otimes b^{\prime}\right)=(-1)^{(n+1)(q+1)} d \theta(c)$ and

$$
(-1)^{(n+1)(q+1)} g \theta(c)=(-1)^{n(q+1)+1} \theta^{\prime \prime}\left(\Delta_{n+1} e_{n} \otimes g(u) \otimes g(v)\right),
$$

by our commutative diagram, and this proves the result.

The analog for the Browder operations of the Adem relations is the following Jacobi identity: let $x \in \mathrm{H}_{q}(K), y \in \mathrm{H}_{r}(\mathrm{~L})$, and $z \in \mathrm{H}_{\mathrm{s}}(\mathrm{K})$; then, under appropriate hypotheses,

$$
\begin{aligned}
(-1)^{(q+n)(s+n)} \lambda_{n}\left(x, \lambda_{n}(y, z)\right) & +(-1)^{(r+n)(q+n)} \lambda_{n}\left(y, \lambda_{n}(z, x)\right) \\
& +(-1)^{(s+n)(r+n)} \lambda_{n}\left(z, \lambda_{n}(x, y)\right)=0,
\end{aligned}
$$

and, if $3=0$ in $\Lambda$ and $q+n$ is odd, $\lambda_{n}\left(x, \lambda_{n}(x, x)\right)=0$. We omit the proof as an easier geometric argument can be obtained for the homology of $(n+1)$-fold loop spaces. This identity, and the identity of (vi) of Proposition 6.3, lead to a notion of $\lambda_{n}$-algebra which generalizes that of Lie algebra (or $\lambda_{0}$-algebra). There is also a notion of restricted $\lambda_{n}$-algebra which is important for the applications. In the case $\Lambda=z_{2}$, the restriction is already present in our algebraic context; it is the last Steenrod operation for an object $(K, \theta) \in \zeta(2, n)$. The following addendum to Proposition 2.3 gives some properties of this operation that are needed in the study of $(n+1)$-fold loop spaces.

Proposition 6.5. Let $(K, \theta) \in \zeta(2, n)$. Let $\xi_{n}=\mathrm{P}_{q+n}: \mathrm{H}_{q}(K) \rightarrow \mathrm{H}_{2 q+n}(K)$. Then

(i) $\quad \xi_{n}(x+y)=\xi_{n}(x)+\xi_{n}(y)+\lambda_{n}(x, y)$, and 
(ii)

$\beta \xi_{n}(x)=(q+n-1) P_{q+n-1}(x)+\lambda_{n}(x, \beta x)$ if $(K, \theta)$ is reduced mod 2

Proof. For (i), if $a$ and $b$ represent $x$ and $y$, then, in $K^{2}$, $(a+b)^{2}=a^{2}+b^{2}+\Delta_{n+1} a b$, and the error term $\Delta_{n+1} e_{n} \otimes a \otimes b$ yields the stated deviation from additivity of $\xi_{n}$. Part (ii) follows from a glance at the proof of (v) of Proposition 2.3.

We now relate the $\lambda_{n}$ to the Bockstein operations on $H(K)$ when $(K, \theta) \in \zeta\left(\pi, n, z_{p}\right)$ is reduced mod p. In contrast to the Steenrod operations, the higher Bocksteins are all of interest.

Proposition 6.7. Let $(K, \theta) \in \zeta\left(\pi, n, Z_{p}\right)$ be reduced mod $p$. Let $x, y \in H(K), \operatorname{deg}(x)=q$. Assume that $\beta_{r}(x)$ and $\beta_{r}(y)$ are defined. Then $\beta_{\mathbf{r}} \lambda_{n}(x, y)$ is defined and, modulo indete rminacy,

$$
\beta_{r} \lambda_{n}(x, y)=\lambda_{n}\left(\beta_{r} x, y\right)+(-1)^{n+q_{\lambda}} \lambda_{n}\left(x, \beta_{r} y\right) \text {. }
$$

Proof. Let $(K, \theta)=\left(\tilde{K} \otimes z_{p}, \tilde{\theta} \otimes z_{p}\right)$. Let $a, b \in K$ be such that their $\bmod p$ reductions $\bar{a}, \bar{b} \in K$ represent $x$ and $y$. We may assume that $d(a)=p^{r} a^{\prime}$ and $d(b)=p^{r} b^{\prime}$; the $\bmod p$ reduction $\bar{a}^{\prime}$ and $\bar{b}^{\prime}$ of $a^{\prime}$ and $b^{\prime}$ represent $\beta_{r}(x)$ and $\beta_{r}(y)$. In $W^{(n)} \otimes \tilde{K}^{2}, d\left(\Delta_{n+1} e_{n} \otimes a \otimes b\right)=(-1)^{n} p^{r} \Delta_{n+1} e_{n} \otimes\left(a^{\prime} \otimes b+(-1)^{q} \otimes_{b} b^{\prime}\right)$. By reduction mod $p$ and a check of signs, this implies the result.

Surprisingly, the following fundamental result appears not to be in the literature, although it is presumably well-known. It allows complete calculation of the mod $p$ homology Bockstein spectral sequence of $Q X=\underline{\lim } \Omega^{n^{n}} S^{n}$ for any space $X$ and, as we shall show later, the $\bmod \mathrm{p}$ cohomology Bockstein spectral sequence of $K(\pi, n)$. Together with the previous result, it also suffices for the computation of the mod p homology Bockstein spectral sequence of $\Omega^{n} S^{n} X, n \geq 1$.

\section{Proposition 6.8 , Let $\mathrm{K}$ be a $\mathrm{Z}$-graded associative differential ring} which is flat as a $Z$-module. Let $K$ have a $u_{1}$-product such that (a) $\quad d\left(a u_{1} b\right)=-d(a) u_{1} b-(-1)^{\operatorname{deg} a} a u_{1} d(b)+a b-(-1)^{\operatorname{deg} a \operatorname{deg} b_{b a}}$ 
and, for case (ii), such that the Hirsch formula (b) holds

(b) $\quad a b u_{1} c=(-1)^{\operatorname{deg} a} a\left(b u_{1} c\right)+(-1)^{\operatorname{deg} b \operatorname{deg} c}\left(a u_{1} c\right) b$.

Let $\beta_{r}$ denote the $r$-th $\bmod p$ Bockstein on $H\left(K \otimes z_{p}\right), \beta_{1}=\beta$. Let $y \in H_{2 q}\left(K \otimes z_{p}\right)$ and assume that $\beta_{r-1}(y)$ is defined, $r \geq 2$. Then $\beta_{r}\left(y^{p}\right)$ is defined and, modulo indeterminacy,

(i) If $\mathrm{p}=2$ and $\mathrm{r}=2, \beta_{2}\left(\mathrm{y}^{2}\right)=\beta(\mathrm{y}) \mathrm{y}+\mathrm{P}_{2 \mathrm{q}} \beta(\mathrm{y})$

(ii) If $p>2$ and $r=2, \beta_{2}\left(y^{p}\right)=\beta(y) y^{p-1}+\sum_{j=1}^{m} j \lambda_{1}\left(\beta(y) y^{j-1}, \beta(y) y^{p-j-1}\right)$

(iii) If $p \geq 2$ and $r \geq 3, \beta_{r}\left(y^{p}\right)=\beta_{r-1}(y) y^{p-1}$.

Proof. Let $b \in K_{2 q}$ be such that its mod $p$ reduction $\bar{b}$ represents $y$. We may assume that $d(b)=p^{r-1} a$, and then $a$ is a cycle whose mod p reduction $\bar{a}$ represents $\beta_{\mathbf{r}-1}(\mathrm{y})$. Clearly we have

$$
\begin{gathered}
d\left(b^{p}\right)=p^{r-1} \sum_{i=1}^{p} b^{i-1} a b^{p-i}, \quad \text { and } \\
d\left(a b^{p-i} u_{1} b^{i-1}\right) \equiv a b^{p-1}-b^{i-1} a b^{p-i} \bmod p^{r-1}, \quad 2 \leq i \leq p .
\end{gathered}
$$

Therefore $d\left(b^{p}+p^{r-1} \sum_{i=2}^{p} a b^{p-i} u_{1} b^{i-1}\right) \equiv p^{r} a b^{p-1} \bmod p^{2 r-2}$.

If $r \geq 3$, then $2 r-2>r$ and part (iii) follows. Thus let $r=2$; we must now take into account the terms arising from $d(b)=p a$ in $d\left(a b^{p-i} u_{1} b^{i-1}\right)$. If $p=2$, then

$$
d\left(b^{2}+2 a u_{1} b\right)=4 a b+4 a y_{1} \text {. }
$$

Since the mod 2 reduction of $a u_{1}$ a represents $P_{2 q} \beta(y)$, this proves (i). Thus assume that $p>2$. Then

$$
\begin{aligned}
& a\left(b^{p}+p \sum_{i=2}^{p} a b^{p-i} u_{1} b^{i-1}\right)=p^{2} a b^{p-1}+p^{2} c+p^{2} c^{\prime}, \quad \text { where } \\
& c=\sum_{i=2}^{p-1} \sum_{j=1}^{p-i} a b^{j-1} a b^{p-i-j} u_{1} b^{i-1} \text { and } \quad c^{\prime}=\sum_{1 \leq j<i \leq p} a b^{p-i} u_{1} b^{j-1} a b^{i-j-1} .
\end{aligned}
$$

By the Hirsch formula, and a separate reindexing of the two resulting sums, we find that 


$$
c=\sum_{2 \leq j<i \leq p}\left[\left(a b^{i-j-1} \cup_{1} b^{j-1}\right) a b^{p-i}-a b^{p-i}\left(a b^{i-j-1} \cup_{1} b^{j-1}\right)\right] .
$$

Therefore if $e=\sum_{2 \leq j<i \leq p} a b^{p-i} u_{1}\left(a b^{i-j-1} u_{1} b^{j-1}\right)$, then

$$
d(e) \equiv-c+\sum_{2 \leq j<i \leq p} a b^{p-i} u_{1}\left(a b^{i-2}-b^{j-1} a b^{i-j-1}\right) \bmod p
$$

Comparing $d(e)$ to $c^{\prime}$, we easily find that

$$
\begin{aligned}
& d\left(b^{p}+p \sum_{i=2}^{p} a b^{p-i} u_{1} b^{i-1}+p^{2} e\right) \equiv p^{2} a b^{p-1}+p^{2} \sum_{i=2}^{p}(i-1) a b^{p-i} u_{1} a b^{i-2} \\
& \equiv p^{2} a b^{p-1}+p^{2} \sum_{j=1}^{m} j\left(a b^{p-j-1} \cup_{1} a b^{j-1}-a b^{j-1} v_{1} a b^{p-j-1}\right) \bmod p^{3} .
\end{aligned}
$$

By Definition 6.2 , this implies (ii) and so completes the proof.

Of course, the terms involving $\lambda_{l}$ in (ii) are zero if $K$ admits a $u_{2}$-product. The general result is needed for second loop spaces. The Hirsch formula is valid for the cochains of a space $[8,16]$, for the chains of a second loop space [10], and for the dual of the bar construction of a cocommutative Hopf algebra [16]. In connection with this formula, we make the following remarks.

Remarks 6.9. Let $\mathrm{K}$ be an associative differential $\mathrm{Z}_{\mathrm{p}}$-algebra, $\mathrm{p}>2$, with a $\cup_{1}$-product which satisfies the Hirsch formula. Define $<>^{\mathrm{P}}: \mathrm{H}_{2 \mathrm{~s}-1}(\mathrm{~K}) \rightarrow \mathrm{H}_{2 \mathrm{sp}-2}(\mathrm{~K})$ as follows. Let $\mathrm{a}_{1}$ represent $\mathrm{x} \in \mathrm{H}_{2 \mathrm{~s}-1}(\mathrm{~K})$ and define $a_{i}=\frac{1}{i} a_{i-1} u_{1} a_{1}$ for $2 \leq i<p$. Then $d\left(a_{i}\right)=\sum_{j=1}^{i-1} a_{j} a_{i-j}$ and $\tilde{a}=\sum_{j=1}^{p-1} a_{j} a_{p-j}$ is a cycle. A computation demonstrates that if $\left\{a_{i} \mid 1 \leq i<p\right\}$ is any set of elements of $K$ such that $a_{1}^{1}$ represents $x$ and $d\left(a_{i}^{\prime}\right)=a_{j}^{\prime} a_{i-j}^{\prime}$ for $2 \leq i<p$, then $\tilde{a}^{\prime}=\sum_{j=1}^{p-1} a_{j}^{\prime} a_{p-j}^{\prime}$ is homologous to $\tilde{a}$. Thus the class of $\tilde{a}$ depends only on $x$, and we define $\langle x\rangle^{P}=\{\tilde{a}\}$. In the applications, it is often the case that if $(K, \theta) \in \zeta(p, p-2)$, then $\langle x\rangle^{P}=-\beta P_{s}(x)$. Kraines [11] has proven this result for the cohomology of spaces, where it reads 
$\langle x\rangle^{P}=-\beta P^{s}(x)$ for $x \in H^{2 s+1}(K)$, and Kochman [10] has proven it for the homology of ite rated loop spaces. A general proof within our algebraic context should be possible, but appears to be difficult.

\section{The category of simplicial $\Lambda$-modules}

We here develop some machinery that will allow us to apply the theory of the previous sections to a large simplicial category $\mathcal{Q} a$. We shall specialize to specific categories of interest in the next section. We assume familiarity with the basic definitions of the theory of simplicial objects and of acyclic models (see, e.g., $[15, \S 1,2,28,29])$. Let $\Lambda$ be a commutative ring, and let $a, \xi a$ and $\& a$ denote the categories of (ungraded) $\Lambda$-modules, positively graded $\Lambda$-complexes, and simplicial $\Lambda$-modules. Let $C: A Q \rightarrow \zeta Q$ be the normalized chain complex functor (for $\mathrm{K} \in \mathbb{\ell} \mathrm{A}, \mathrm{C}(\mathrm{K}$ ) is the quotient of $\mathrm{K}$, regarded as a chain complex with $d=\sum(-1)^{i} d_{i}$, by the subcomplex generated by the degenerate simplices). Define $H_{*}(K)=H(C(K))$ and $H^{*}(K)=H(C *(K))$, where $C^{*}(K)=\operatorname{Hom}_{\Lambda}(C(K), \Lambda)$ is given the differential $\delta(f)(k)=(-1)^{q+1} f(d k)$ for $f \in C^{q}(K)$ and $k \in C_{q+1}(K)$. The following key lemma is based on ideas of Dold [ 5 ].

Lemma 7.1. Let $\pi$ be a subgroup of $\Sigma_{\mathbf{r}}$ and let $W$ be a $\Lambda \pi$-free resolution of $\Lambda$ such that $W_{0}=\Lambda \pi$ with $\Lambda \pi$-generator $\mathrm{e}_{0} \cdot$ Let $\mathrm{K}_{1}, \ldots, \mathrm{K}_{\mathrm{r}} \in 8 \mathrm{Sa} ;$ then the re exists a morphism of $\Lambda$-complexes $\Phi: w \otimes C\left(K_{1} \stackrel{x}{\otimes} \ldots \otimes^{x} \mathrm{~K}_{r}\right) \longrightarrow w \otimes C\left(K_{1}\right) \otimes \ldots \otimes c\left(K_{r}\right)$

which is natural in the $K_{i}$ and satisfies the following properties: 
(i) For $\sigma \in \pi$, the following diagram is commutative:

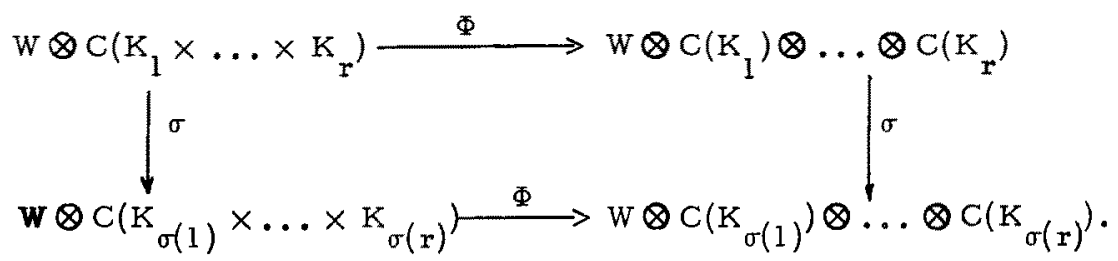

(ii) $\Phi$ is the identity homomorphism on $\mathrm{W} \otimes \mathrm{C}_{\mathrm{o}}\left(\mathrm{K}_{1} \times \ldots \times \mathrm{K}_{\mathrm{r}}\right)$.

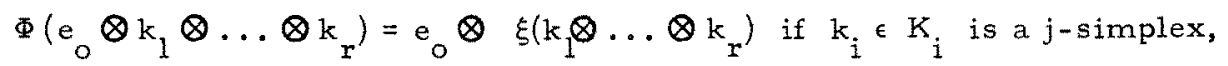

where $\xi: C\left(K_{1} \times \ldots \times K_{r}\right) \rightarrow C\left(K_{1}\right) \otimes \ldots \otimes C\left(K_{r}\right)$ is the Alexander-Whitney map.

(iv) $\Phi\left(W \otimes C_{j}\left(K_{1} \times \ldots \times K_{r}\right)\right) \subset \sum_{k \leq r j} w \otimes\left[C\left(K_{1}\right) \otimes \ldots \otimes C\left(K_{r}\right)\right]_{k} \cdot$

Moreover, any two such $\Phi$ are naturally equivariantly homotopic

Proof. Since $\left(K \times L_{j}=K_{j} \otimes L_{j}\right.$, formulas (ii) and (iii) make sense.

Write $A_{j}=C_{j}\left(K_{1} \times \ldots \times K_{r}\right)$ and $B_{j}=\left[C\left(K_{1}\right) \otimes \ldots \otimes C\left(K_{r}\right)\right]_{j}$. We construct

$\Phi$ on $W_{i} \otimes A_{j}$ by induction on $i$ and for fixed $i$ by induction on $j$. Formula (ii) defines $\Phi$ for $j=0$ and all $i$ and formulas (i) and (iii) define $\Phi$ for $i=0$ and all $j$. Thus let $i \geq 1$ and $j \geq 1$ and assume that $\Phi$ is defined for $i^{\prime}<i$ and for the given $i$ and $j^{\prime}<j$. Choose a $\Lambda \pi$-basis $\left\{w_{k}\right\}$ for $w_{i}$. It suffices to define $\Phi$ on $w \otimes x$ for $w \in\left\{w_{k}\right\}$ and $x \in A_{j}$, since $\Phi$ can then be uniquely extended to all of $W_{i} \otimes A_{j}$ by (i). Let $\Lambda \Delta[j]$ denote the free simplicial $\Lambda$-module generated by the standard simplicial $j$-simplex $[15, \mathrm{p}, 14]$. Then the functor $w \otimes A_{j}$ is represented by the $r$-fold Cartesian product $\Lambda \Delta[j]^{r}$, and $W \otimes B\left(\Lambda \Delta[j]^{r}\right)$ is acyclic. Therefore $\Phi\left(w \otimes \Delta_{j} \otimes \ldots \otimes \Delta_{j}\right)$ can be defined by choosing a chain whose boundary is $\Phi \mathrm{d}\left(\mathrm{w} \otimes \Delta_{\mathrm{j}} \otimes \ldots \otimes \Delta_{j}\right)$, and $\Phi$ can be carried over to arbitrary $w \otimes k_{1} \otimes \ldots \otimes k_{r}$ by representability. Now (i), (ii), and (iii) are clearly satisfied and (iv) follows from the fact that $C_{k}(\Lambda \Delta[j])=0$ for $k>j$. The proof that $\Phi$ is unique up to natural equivariant homotopy is equally simple. 
Remarks 7.2. Define $\Psi: \mathrm{W} \otimes \mathrm{C}\left(\mathrm{K}_{1}\right) \otimes \ldots \otimes \mathrm{C}\left(\mathrm{K}_{\mathrm{r}}\right) \rightarrow \mathrm{W} \otimes \mathrm{C}\left(\mathrm{K}_{1} \times \ldots \times \mathrm{K}_{\mathrm{r}}\right)$ by $\Psi=1 \otimes \eta \quad$ where $\eta: C\left(K_{1}\right) \otimes \ldots \otimes C\left(K_{r}\right) \rightarrow C\left(K_{1} \times \ldots \times K_{r}\right)$ is the shuffle map. Since $\eta$, unlike $\xi$, is commutative, $\Psi$ is equivariant. By an easy acyclic models proof, $\Phi \Psi$ and $\Psi \Phi$ are equivariantly homotopic to the respective identity maps.

We shall only be interested in the case $K_{1}=\ldots=K_{r}$; here $\Phi: W \otimes C\left(K^{r}\right) \longrightarrow W \otimes C(K)^{r}$ is a natural morphism of $\Lambda \pi$-complexes. The general case was required in the proof in order to have the representability of the functors $A_{j}$. Starting with objects of the following category $19 a$, we shall use $\Phi$ to obtain diagonal approximations and so to pass to the category $P(\pi, \infty, \Lambda) \subset \zeta(\pi, \infty, \Lambda)$ defined in Definitions 2.1

Definitions 7.3. Let $19 a$ denote the following category. The objects of $19 Q$ are pairs $(K, D)$ where $K \in \& Q$ and $D: K \rightarrow K \times K$ is a morphism.in $\& Q$ such that $(D \times 1)=(1 \times D) D$ and $t D=D$, where $t(x \otimes y)=y \otimes x$. The morphisms

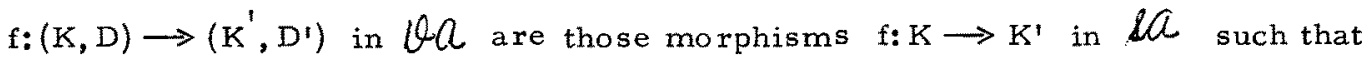
$(f \times f) D=D^{\prime} f$.

Each $K \in \& Q$ admits the natural diagonal $D(k)=k \otimes k$, and $\$ C$ is the reby embedded as a full subcategory of $h l a$. However, an object $\mathrm{K} \in \mathrm{SC}$ may admit other interesting diagonals. For example, if $\mathrm{K}$ is a simplicial cocommutative coassociative $\Lambda$-coalgebra, then the coproduct $\psi: K \rightarrow \mathrm{K} \times \mathrm{K}$ is a permissible diagonal; that is, $(\mathrm{K}, \psi) \in \mathbb{W}$. The following remarks will be of use in the study of relative and reduced cohomology.

Remarks 7.4. (i) If $\mathrm{L} C \mathrm{~K}$ in $\& A$, define $\mathrm{H}_{*}(\mathrm{~K}, \mathrm{~L})=\mathrm{H}(\mathrm{C}(\mathrm{K} / \mathrm{L}))$ and $H^{*}(K, L)=H\left(C^{*}(K / L)\right)$. If $(K, D) \in \operatorname{lQC}$ and $D(L)$ is contained in $L \times K+K \times L$, then $\mathrm{K} / \mathrm{L}$ admits the diagonal $\overline{\mathrm{D}}$ induced from the composite $\mathrm{K} \stackrel{\mathrm{D}}{\longrightarrow} \mathrm{K} \times \mathrm{K} \stackrel{\pi \times \pi}{\longrightarrow} \mathrm{K} / \mathrm{L} \times \mathrm{K} / \mathrm{L}$, where $\pi: \mathrm{K} \rightarrow \mathrm{K} / \mathrm{L}$ is the projection, and then $\pi$ is a morphism in hla. 
(ii) Let $\tilde{\Lambda}=\Lambda \Delta[0]$ e Sa, thus $\tilde{\Lambda}_{n}=\Lambda$ for $n \geq 0$, each $d_{i}$ and $s_{i}$ is the identity, and $C(\tilde{\Lambda})=C_{0}(\tilde{\Lambda})=\Lambda$. Give $\tilde{\Lambda}$ the natural diagonal. We say that $(K, D) \in k \infty$ is unital if we are given a monomorphism $v: \tilde{\Lambda} \rightarrow K$ in $10 a$ and an epimorphism $\varepsilon: \mathrm{K} \rightarrow \tilde{\Lambda}$ in $\mathcal{L}$ such that $\varepsilon v=1$ and $(\varepsilon \times 1) \mathrm{D}=(1 \times \varepsilon) \mathrm{D}$ (where $\tilde{\Lambda} \times K=K=K \times \tilde{\Lambda})$. If $(K, D)$ is unital and $I K=\operatorname{Ker} \varepsilon$, then $K=v(\tilde{\Lambda}) \oplus I K$ and for $k \in I K, D(k)=k \otimes v(1)+v(1) \otimes k+\bar{D}(k)$, where $\bar{D}(k) \in I K \times I K$. Clearly, $(\mathrm{IK}, \overline{\mathrm{D}})$ is isomorphic to $(\mathrm{K} / v(\tilde{\Lambda}), \overline{\mathrm{D}})$ in $\mathrm{Na}$.

If $(K, D) \in L N a$, then $C^{*}(K)$ is an associative differential $\Lambda$-algebra, with cup product defined as the composite (1) $u: \mathrm{C}^{*}(\mathrm{~K}) \otimes \mathrm{C}^{*}(\mathrm{~K}) \stackrel{\alpha}{\longrightarrow}[\mathrm{C}(\mathrm{K}) \otimes \mathrm{C}(\mathrm{K})]^{*} \stackrel{\xi^{*}}{\longrightarrow} \mathrm{C}^{*}(\mathrm{~K} \times \mathrm{K}) \stackrel{\mathrm{D}^{*}}{\longrightarrow} \mathrm{C}^{*}(\mathrm{~K})$. Here $\alpha$ is the natural map, $\alpha(x \otimes y)(k \otimes \ell)=(-1)^{\operatorname{deg} y \operatorname{deg} k} x(k) y(\ell)$, and $\xi$ is the Alexander-Whitney map. If $(K, D)$ is unital, then $C^{*}(K)$ is unital (via $\nu^{*}$ ) and augmented (via $\varepsilon^{*}$ ).

We now define a functor $\Gamma: 19 a \rightarrow P(\pi, \infty, \Lambda)$ and then show how to use $\Gamma$ to apply our general theory to $H^{*}(K)$ for $(K, D) \in \omega Q$ in the case $\Lambda=Z_{p}$.

Definitions 7.5. Let $(K, D) \in 100$ and write $D$ for the iterated diagonal $K \rightarrow K^{r}$. Let $\pi \subset \Sigma_{r}$ and let $W$ be a $\Lambda \pi$-free resolution of $\Lambda$ with $W_{0}=\Lambda \pi$. Define $\Delta: \mathrm{W} \otimes \mathrm{C}(\mathrm{K}) \rightarrow \mathrm{C}(\mathrm{K})^{\mathrm{r}}$ to be the composite

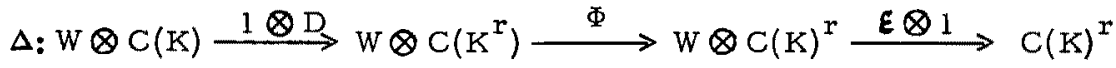

Let $\alpha: C^{*}(K)^{r} \rightarrow\left[C(K)^{r}\right]^{*}$ be the natural map and define a $\Lambda \pi$-morphism $\theta: W \otimes C^{*}(K)^{r} \rightarrow C^{*}(K)$ by the formula

(3) $\quad \theta(w \otimes x)(k)=(-1)^{\operatorname{deg} w \operatorname{deg} x} \alpha(x)(\Delta(w \otimes k)), w \in W, x \in C^{*}(K)^{r}, k \in C(K)$. Since $\theta$ may be defined for $\pi=\Sigma_{r}$ and then factored through $j: W \rightarrow V$ as in Definition 2.1, and the resulting composite is $\Lambda \pi$-homotopic to the original $\theta$ defined in terms of $W, \theta$ satisfies condition (ii) of Definition 2. 1. By Lemma 7.1, formula (3) specializes to give

$$
\theta\left(e_{0} \otimes x\right)=D^{*} \xi^{*} \alpha(x) \text { for any } x \in C^{*}(K)^{r} \text {, and }
$$


(5) $\quad \theta(w \otimes x)=\varepsilon(w) D^{*} \alpha(x)$ if $x \in C^{\circ}(K)^{r}$ and $w \in W$.

By (1) and (4), $\theta$ satisfies condition (i) of Definition 2.1. Since $\theta$ is natural on

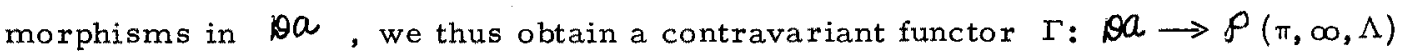
by letting $\Gamma(K, D)=\left(C^{*}(K), \theta\right)$ on objects and $\Gamma(f)=C^{*}(f)$ on morphisms. By $(5)$, if $(K, D)$ is unital in 190 then $\Gamma(K, D)$ is unital in $p(\pi, \infty, \Lambda)$. If $\Lambda=Z_{p}$, is cyclic of order $p$, and $(K, D)=\left(\tilde{K} \otimes z_{p}, \tilde{D} \otimes z_{p}\right)$ where $\tilde{K}$ is a $Z$-free simplicial $Z$-module, we agree to choose $\theta$ for $K$ to be the mod $p$ reduction of $\theta$ for $\tilde{K}$; then $\Gamma(K, D)$ is reduced $\bmod p\left(\right.$ since $C(\tilde{K})$ is $Z$-free and therefore $C^{*}(\tilde{K})$ is Z-flat, as required by Definition 2.1).

Observe that, by Definition 6.1 , we now have $u_{j}$-products in $C^{*}(K)$ for any $(K, D) \in 19 a$. When $\Lambda=Z_{p}$, the results of Proposition 2.3 will clearly apply to the Steenrod operations $P^{S}$ defined on the cohomology of objects $(K, D) \in \mathfrak{N O}$. If $(K, D)$ and $\left(L, D^{\prime}\right)$ are objects of $19 a$, then $K \times L$ admits the diagonal $\tilde{D}=(1 \times t \times 1)\left(D \times D^{\prime}\right)$; if $D^{\prime}$ and $D^{\prime}$ are the natural diagonals, then so is $\tilde{D}$. Thus $\left(C^{*}(\mathrm{~K} \times L), \theta\right)$ is defined in $\quad \zeta(\pi, \infty, \Lambda)$. The following lemma compares $\left(C^{*}(K \times L), \theta\right)$ to $\left(C^{*}(K) \otimes C^{*}(L), \tilde{\theta}\right)$ and will imply the applicability of the external Cartan formula to $H^{*}(K \times L)$ when $\Lambda=Z_{p}$.

Lemma 7.6. For any objects $(K, D)$ and $\left(L, D^{\prime}\right)$ in $19 Q$, the following diagram is $\Lambda \pi$-homotopy commutative

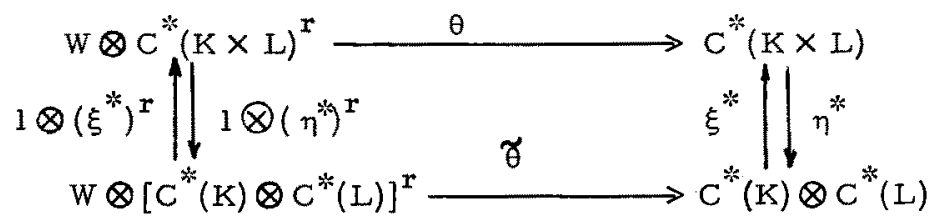

That is, $\eta^{*}$ and $\xi^{*}$ are morphisms in the category $\zeta(\pi, \infty, \Lambda)$.

Proof. By the definitions of $\theta$ and $\tilde{\theta}=(\theta \otimes \theta)(1 \otimes T \otimes 1)(\psi \otimes U)$, it suffices to show that the following diagram is $\Lambda \pi$-homotopy commutative: 


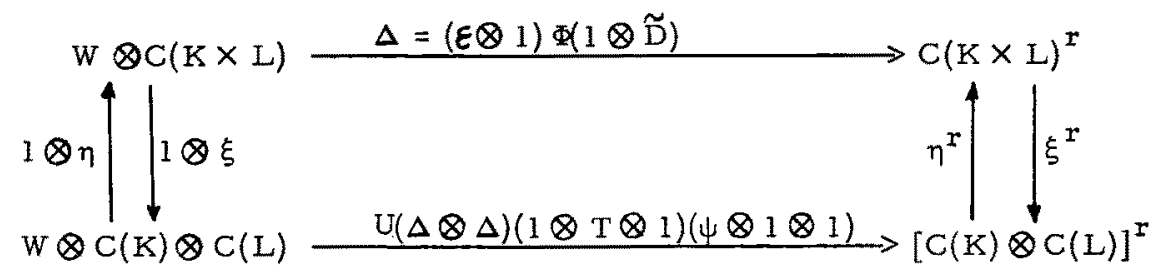

Since $\left(1 \otimes D \otimes 1 \otimes D^{\prime}\right)(1 \otimes T \otimes 1)(\psi \otimes 1 \otimes 1)=(1 \otimes T \otimes 1)(\psi \otimes 1 \otimes 1)\left(1 \otimes D \otimes D^{\prime}\right)$, if we let $\phi=(\varepsilon \otimes 1) \Phi$ and let $\mathrm{u}: \mathrm{K}^{\mathrm{r}} \times \mathrm{L}^{\mathrm{r}} \rightarrow(\mathrm{K} \times \mathrm{L})$ be the evident shuffle, so that $\tilde{D}=u\left(D \times D^{\prime}\right)$, then this diagram becomes

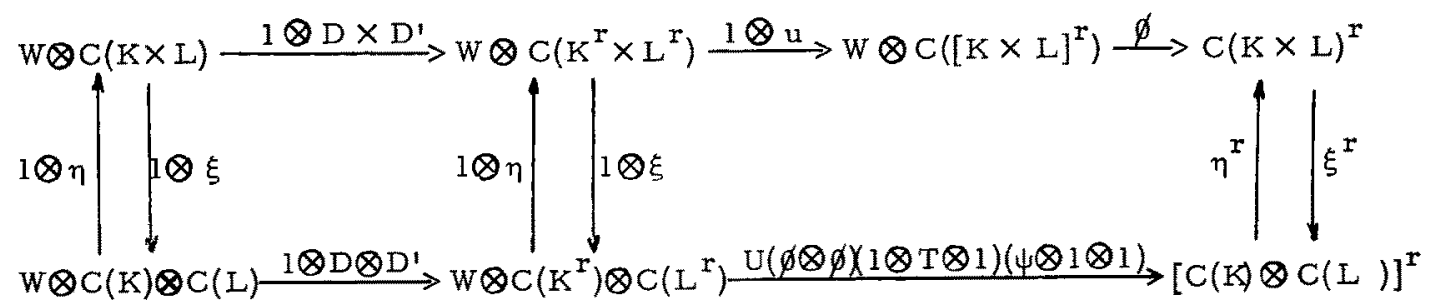

The left-hand square commutes by the naturality of $\eta$ and $\xi$. Since the diagonals are not involved int he right-hand square, we can prove that it commutes up to $\Lambda \pi$-homotopy by an acyclic models argument, with $K^{r}$ and $L^{r}$ replaced by $K_{1} \times \ldots \times K_{r}$ and $L_{1} \times \ldots \times L_{r}$ so as to have domains given by representable functors for fixed $w \in W$. On zero simplices, the diagram commutes for any $w \in W$ and on $e_{0} \in W$, as the simplices vary, the diagram is $\Lambda$-homotopy commutative by a standard acyclic models argument. This starts the inductive construction of the desired homotopies, and the proof is completed precisely as was the proof of Lemma 7.1 .

Corollary 7.7. If $(K, D) \in Q a$, then $\Gamma(K, D)$ is a Cartan object of $\zeta(\pi, \infty, \Lambda)$.

Proof. Since $\mathrm{D}: \mathrm{K} \rightarrow \mathrm{K} \times \mathrm{K}$ is commutative and as sociative, it is a morphism in $19 a$. Therefore the cup product ( 1 ) is a morphism in $\zeta(\pi, \infty, \Lambda)$, as required. 
Lemma 7.8. If $(K, D) \in N Q, \Lambda=Z_{p}$, then $\Gamma(K, D)$ is an Adem object of $\zeta(p, \infty)$.

Proof. In the notations of Definition 4.1 (with $\mathrm{Y}_{\mathrm{o}}=\mathrm{Z}_{\mathrm{p}} \Sigma_{\mathrm{p}}{ }^{2}$ ), it suffices to prove that the following diagram is T-homotopy commutative:

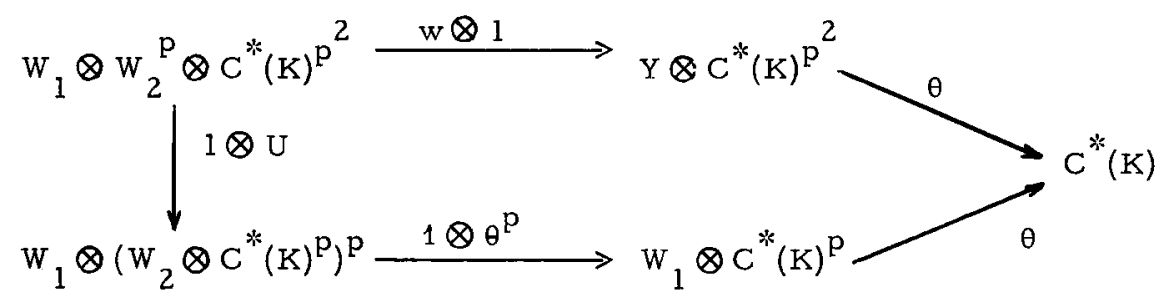

All maps $\theta$ are as defined in Definition 7.5; by dualization, it suffices to prove that the following diagram is $T$-homotopy commutative

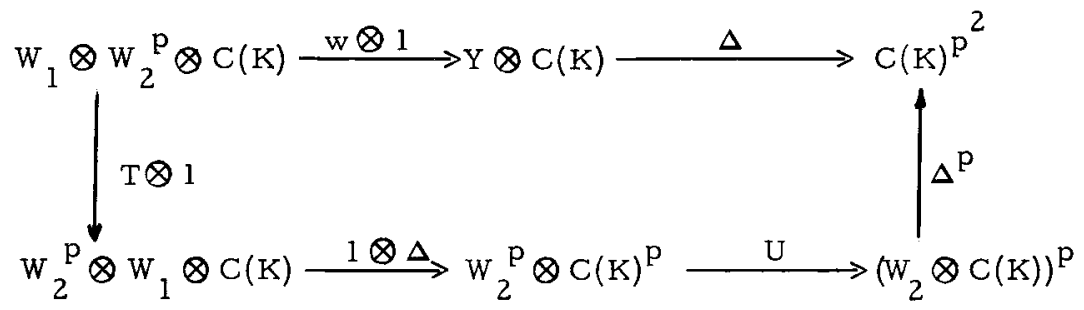

Let $\phi=(\varepsilon \otimes 1) \Phi \quad$ and define $\alpha=\phi(\mathrm{w} \otimes 1): \mathrm{w}_{1} \otimes \mathrm{w}_{2}^{\mathrm{P}} \otimes \mathrm{C}\left(\mathrm{K}^{\mathrm{P}^{2}}\right) \longrightarrow \mathrm{C}(\mathrm{K})^{\mathrm{p}^{2}}$. Since $\Delta=\phi(1 \otimes \mathrm{D}), \Delta(\mathrm{w} \otimes 1)=\alpha(1 \otimes 1 \otimes \mathrm{D}), \mathrm{D}: \mathrm{C}(\mathrm{K}) \rightarrow \mathrm{C}\left(\mathrm{KP}^{2}\right)$. By the naturality of $\phi$, the following diagram is commutative:

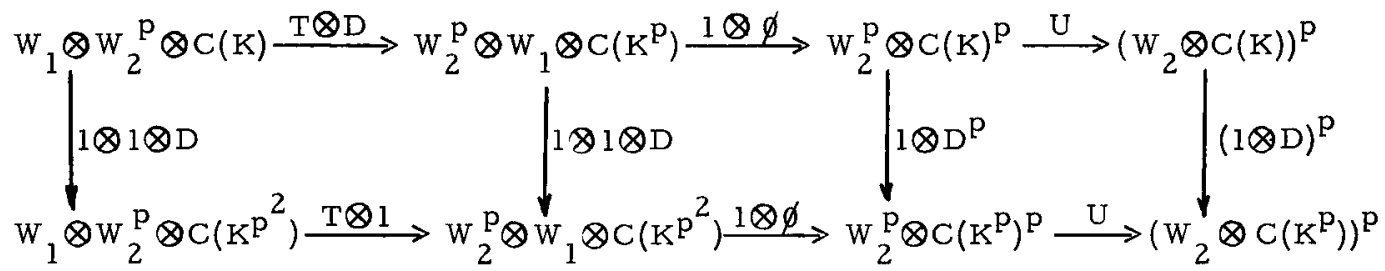

Let $\beta=\phi^{\mathrm{p}} \mathrm{U}(1 \times \phi)(\mathrm{T} \times 1): \mathrm{W}_{1} \times \mathrm{W}_{2}^{\mathrm{p}} \times \mathrm{C}\left(\mathrm{K}^{\mathrm{p}^{2}}\right) \longrightarrow \mathrm{C}(\mathrm{K})^{\mathrm{P}^{2}} \cdot$ By the diagram above,

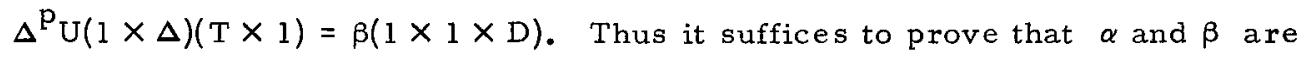
T-homotopic. Since $\alpha$ and $\beta$ do not involve the diagonal, this can easily be shown by acyclic models precisely as in our previous proofs. 
The following theorem summarizes properties of the Steenrod operations that are valid for arbitrary objects of the category $\ominus Q, \Lambda=z_{p^{*}}$ Of course, we use the notations of section 5 since we are dealing with cohomology.

Theorem 7.9. Let $(K, D) \in \mathbb{1 9 a}, \Lambda=Z_{\mathrm{p}^{*}}$ Then the re exist natural homomorphisms $\mathrm{P}^{\mathrm{s}}$ and, if $\mathrm{p}>2, \beta \mathrm{P}^{\mathrm{s}}$ defined on each $\mathrm{H}^{\mathrm{q}}(\mathrm{K})$; $\operatorname{degree}\left(\mathrm{P}^{\mathrm{s}}\right)=\mathrm{s}$ if $\mathrm{p}=2$ and $\operatorname{deg}\left(\beta^{\varepsilon} \mathrm{p}^{\mathrm{s}}\right)=2 \mathrm{~s}(\mathrm{p}-1)+\varepsilon, \varepsilon=0$ or 1 , if $\mathrm{p}>2$. These cohomology operations on $10 a$ satisfy the properties

(i) $\beta^{\varepsilon} \mathrm{P}^{\mathrm{s}}=0$ if $\mathrm{s}<0$ or if $\mathrm{p}=2 \quad(\varepsilon=0)$ and $\mathrm{s}>\mathrm{q}$ or if $\mathrm{p}>2$ and $2 \mathrm{~s}+\varepsilon>\mathrm{q}$.

(ii) $P^{s}(x)=x^{2}$ if $p=2$ and $s=q ; P^{s}(x)=x^{p}$ if $p>2$ and $2 s=q$

(iii) If $(K, D)=\left(\tilde{K} \otimes Z_{p}, \tilde{D} \otimes z_{p}\right)$, where $\tilde{K}$ is $Z$-free, then $\beta P^{s-1}=s P^{s}$ if $p=2$ and $\beta \mathrm{P}^{\mathrm{S}}$ is the composition of $\beta$ and $\mathrm{P}^{\mathrm{s}}$ if $\mathrm{p}>2$.

(iv) $\mathrm{P}^{\mathrm{s}}=\sum \mathrm{P}^{i} \otimes \mathrm{P}^{\mathrm{s}-\mathrm{i}}$ and $\beta \mathrm{P}^{\mathrm{s}}=\sum\left(\beta \mathrm{P}^{i} \otimes \mathrm{P}^{\mathrm{s}-\mathrm{i}}+\mathrm{P}^{i} \otimes \beta \mathrm{P}^{\mathrm{s}-\mathrm{i}}\right)$ on $\mathrm{H}^{*}(\mathrm{~K} \times L)$; the internal Cartan formula is satisfied in $H^{*}(K)$

(v) If $\mathrm{f}: \mathrm{K}^{\prime} \rightarrow \mathrm{K}$ and $\mathrm{g}: \mathrm{K} \rightarrow \mathrm{K}^{\prime \prime}$ are morphisms in 190 such that $\mathrm{gf}=0$, then $\sigma \beta^{\varepsilon} P^{s}=(-1)^{\varepsilon} \beta^{\varepsilon} P^{s} \sigma$, where $\sigma: H^{q}\left(K^{\prime \prime}\right) \rightarrow H^{q-1}\left(K^{\prime}\right)$ is the suspension associated with $\mathrm{C}^{*}\left(\mathrm{~K}^{\prime \prime}\right) \rightarrow \mathrm{C}^{*}(\mathrm{~K}) \rightarrow \mathrm{C}^{*}\left(\mathrm{~K}^{\prime}\right)$.

(vi) If $L \subset K$ and $D(L) \subset L \times L$, then $\delta \beta^{\varepsilon} P^{s}=(-1)^{\varepsilon} \beta^{\varepsilon} P^{s} \delta$ where $\delta: H^{q}(L) \rightarrow H^{q+1}(K, L)$ is the connecting homomorphism.

(vii) The $\beta^{\varepsilon} \mathrm{P}^{\mathrm{s}}$ satisfy the Adem relations as stated in Corollary 5.1.

Proof. For (i), we must prove that $\beta^{\varepsilon_{P}} s=0$ for $s<0$ (the rest is the convention $\mathrm{e}_{\mathrm{i}}=0$ for $\mathrm{i}<0$ ); by formulas (5.1) and (5.2), it suffices to show that $D_{i}(x)=0$ for $i>(p-1) q, \operatorname{deg}(x)=q$. By (3) of Definition 7.5, it suffices to show that $\Delta\left(e_{i} \otimes k\right)=0$ for $k \in C_{p q-i}(K)$. Now $\Delta=(\varepsilon \otimes 1) \Phi(1 \otimes D)$ and, by (iv) of Lemma 7.1 , if $i>(p-1) q$, then

$$
\Phi\left(e_{i} \otimes D(k)\right) \epsilon \sum_{j<p q} W_{p q-j} \otimes[C(K)]_{j}^{p} c \operatorname{Ker}(\varepsilon \otimes 1) .
$$

(ii) and (iii) follow from Proposition 2.3; (iv) follows from Corollary 2.7, Lemma 7.6, and Corollary 7.7; (v) and (vi) follow from Theorem 3.3, noting for 
(vi) that the suspension associated with $C^{*}(K / L) \rightarrow C^{*}(K) \rightarrow C^{*}(L)$ is the inverse additive relation to the connecting homomorphism $\delta ;$ (vii) follows from Theorem 4.7 and Lemma 7.8.

By Theorem 3.4, the Kudo transgression theorem applies to appropriate spectral sequences involving objects of $10 Q$ and, under the hypotheses of (iii) of the theorem, Proposition 6.8 applies to compute the higher Bocksteins on p-th powers of elements of $H^{*}(K),(K, D) \in \theta a$. In the next section, we shall show how to compoute $\mathrm{P}^{\circ}$ for arbitrary objects $(K, D) \in \hat{\Theta} \alpha$ and shall give non-trivial examples to show that $\mathrm{P}^{0} \neq 1$ in general.

\section{Simplicial sets and simplicial restricted Lie algebras}

We shall here obtain the Steenrod operations on the cohomology of topological spaces, simplicial sets, and simplicial restricted Lie algebras, and shall consider the evaluation of $\mathrm{P}^{\circ}$ on $\mathrm{H}^{*}(\mathrm{~K})$ for any $(K, D) \in i 0 Q, \Lambda=\mathrm{Z}_{\mathrm{p}}$,

Let $\&$ denote the category of simplicial sets. For $K \in \mathbb{\&}$, let $\tilde{K}$ denote the free simplicial Abelian group generated by $K$. Let $\Lambda$ be a commutative ring and define a functor $\mathrm{A}: \mathcal{L} \rightarrow \mathfrak{B Q}$ by letting $\mathrm{A}(\mathrm{K})=\breve{\mathrm{K}} \otimes \Lambda$ with its natural diagonal $D$; here $19 a$ is as defined in Definition 7.3 and $D$ is induced from the diagonal $k \rightarrow(k, k)$ on $k$. Composing $A$ with $\Gamma$ of Definition 7.5 , we obtain a functor $\Gamma A:, \mathcal{Q} \rightarrow P(\pi, \infty, \Lambda)$ for any $\pi \subset \Sigma_{r}$. Let $\mathcal{J}$ denote the category of topological spaces and let $\mathrm{S}: \mathrm{T} \rightarrow \&$ be the total singular complex functor. Then IAS: $J \rightarrow P(\pi, \infty, \Lambda)$ is defined. If $(K, L)$ is a simplicial pair, define $A(K, L)=\tilde{K} / \tilde{L} \otimes \Lambda$. Then $\Gamma A$ is defined on the category $\tilde{H}_{2}$ of simplicial pairs and $\Gamma A S$ is defined on the catetogy $J_{2}$ of topological pairs. Sinc e the normalized cochains with coefficients in $\Lambda$ of a simplicial pair $(K, L)$ and of a topological pair $(X, Y)$ may be defined as $C^{*}(K, L)=C^{*}(\tilde{K} / \tilde{L} \otimes \Lambda)$ and $C^{*}(X, Y)=C^{*}(S X, S Y)$, the results of the previous section apply to the cohomology 
of simplicial and topological pairs.

For the remainder of this section, we take $\Lambda=z_{p}$ and we let $\pi$ be cyclic of order p. Via $\Gamma A: \not_{2} \rightarrow P(p, \infty)$, we have Steenrod operations $P^{s}, s \geq 0$, on $H^{*}(K, L)$ for all $(K, L) \in \&_{2}$, hence on $H^{*}(X, Y)$ for all $(X, Y) \in T_{2}$. Of course, if $p=2, \mathrm{P}^{\mathrm{s}}$ is usually denoted by $\mathrm{Sq}^{\mathrm{s}}$. Theorem 7.9 gives all of the standard properties of the $P^{S}$ except $P^{\circ}=1$, We now show that $P^{\circ}=1$ follows from the previously obtained properties of the $P^{S}$.

Proposition 8.1. $\quad P^{\circ}$ is the identity operation and, if $p=2, P^{1}$ is the Bockstein operation on the cohomology of simplicial (or topological) pairs.

Proof. Since $\beta P^{\circ}=P^{l}$ if $p=2$, it suffices to prove that $P^{\circ}=1$, If $(K, L) \in \mathcal{L}_{2}, L$ non-empty, then $H^{*}(K, L)=H^{*}(K / L, P)$, where $P$ is a point complex. Thus it suffices to prove that $P^{\circ}(x)=x$ for $x \in \tilde{H}^{n}(K)=H^{n}(K, P)$, since the result for L empty will follow trivially. If $K^{(n)}$ is the $n$-skeleton of $K$, then $\tilde{H}^{n}(K) \rightarrow \tilde{H}^{n}\left(K^{(n)}\right)$ is a monomorphism, and we may thus as sume that $K=K^{(n)}$. Then, by the Hopf Theorem [24,p.431], there exists $f: K \rightarrow S^{n}$ such that $f^{*}\left(i_{n}^{*}\right)=x$, where $i_{n}^{*} \in \tilde{H}^{n}\left(S^{n}\right)$ is the fundamental class of the simplicial $n$-sphere. It therefore suffices to prove that $P^{0}\left(i_{n}^{*}\right)=i_{n}^{*}$. Now for any $K$, the suspension isomorphism $\mathrm{S}^{*}: \mathrm{H}^{\mathrm{q}+1}(\mathrm{SK}) \longrightarrow \tilde{H}^{\mathrm{q}}(\mathrm{K})$ may be defined as the composite $\mathrm{H}^{\mathrm{q}+\mathrm{l}}(\mathrm{SK}) \longrightarrow \mathrm{H}^{\mathrm{q}+1}(\mathrm{CK}, \mathrm{K}) \longrightarrow \tilde{\mathrm{H}}^{\mathrm{q}}(\mathrm{K})$, where $\mathrm{CK}$ is the simplicial cone of $\mathrm{K}$, hence $S^{*}$ commutes with the $P^{s}$. Since $S^{*}\left(i_{n}^{*}\right)=i_{n-1}^{* *}$ for $n \geq 1$ and $P^{\circ}\left(i_{0}^{*}\right)=\left(i_{0}^{*}\right)^{p}=i_{0}^{*} \quad$ (where $i_{0}^{*}$ generates $\left.\tilde{H}\left(S^{o}\right)=Z_{p}\right)$, this proves the result.

We now use the fact that $P^{O}=1$ on the cohomology of simplicial sets to show how to compute $\mathrm{P}^{\mathrm{O}}$ on $\mathrm{H}^{*}(\mathrm{~K})$ for any object $(K, D) \in \mathcal{D Q}$. In fact, we have the following addendum to Lemma 7,1 when $W$ is the canonical $Z_{p} \pi$-free resolution of $\mathrm{Z}_{\mathrm{p}}$.

Lemma 8.2. Let $K_{i} \in \ell Q, i \leq i \leq p$, and let $k_{i}$ be a $q$-simplex of $K_{i}$. Then, for any $\Phi: W \otimes C\left(K_{1} \times \ldots \times K_{p}\right) \rightarrow W \otimes C\left(K_{1}\right) \otimes \ldots \otimes C\left(K_{p}\right)$ which satisfies 
satisfies the conclusions of Lemma 7.1 ,

$$
(E \otimes 1) \Phi\left(e_{q(p-1)} \otimes k_{1} \otimes \ldots \otimes k_{p}\right)=(-1)^{m q}(-q)^{-1} k_{1} \otimes \ldots \otimes k_{p},
$$

where $v(-\mathrm{q})=1$ if $\mathrm{p}=2$ and $v(-2 \mathrm{j}+\varepsilon)=(-1)^{\mathrm{j}}(\mathrm{m} !)^{\varepsilon}, \varepsilon=0$ or 1 , if $\mathrm{p}>2$.

Proof. Let $\Delta_{\mathrm{q}}$ be the fundamental q-simplex of $\Lambda \Delta[\mathrm{q}]$. Since $\Delta_{\mathrm{q}}$ is a $Z_{p}$-basis for $C_{q}(\Lambda \Delta[q])$, we clearly have that

(i) $\Phi\left(e_{q(p-1)} \otimes \Delta_{q} \otimes \ldots \otimes \Delta_{q}\right) \equiv v_{o} \otimes \Delta_{q} \otimes \ldots \otimes \Delta_{q} \bmod \operatorname{Ker}(\varepsilon \otimes 1), \quad \gamma \in z_{p} \pi$.

By the naturality of $\Phi$ (or by the proof of Lemma 7.1 ), (i) implies

$$
(\varepsilon \times 1) \Phi\left(e_{q(p-1)} \otimes k_{1} \otimes \ldots \otimes k_{p}\right)=\epsilon(\gamma) k_{1} \otimes \ldots \otimes k_{p} \text { for any } k_{i} \in\left(K_{i}\right) q_{2}
$$

To evaluate $\varepsilon(\gamma)$, let $i_{q} \in C_{q}\left(S^{q}\right)$ represent the fundamental class of $H_{q}\left(S^{q}\right)$; we may take $S^{q}=\Delta[q] / \dot{\Delta}[q]$ so that $i_{q}$ is a basis for $C_{q}\left(S^{q}\right)$ and $i_{q}^{*} \in C^{q}\left(S^{q}\right)$ is well-defined. By (ii) and $D\left(i_{q}\right)=i_{q} \otimes \ldots \otimes i_{q}$, Definition 7.5 gives

(iii) $\quad \theta\left(e_{q(p-1)} \otimes i_{q}^{* p}\right)\left(i_{q}\right)=\alpha\left(i_{q}^{* p}\right)\left[(\varepsilon \otimes 1) \Phi\left(e_{q(p-1)} \otimes i_{q}^{p}\right)\right]=(-1)^{m q} \varepsilon(\gamma)$.

Since $P^{\circ}\left\{i_{q}^{*}\right\}=v(-q) D_{q(p-1)}\left\{i_{q}^{*}\right\}=i_{q}^{*}, \quad v(-q) \theta\left(e q(p-1) \otimes\left(i_{q}^{*} p\right)=i_{q}^{*}\right.$. Thus $(-1)^{\mathrm{mq}} \nu(-q) \varepsilon(\gamma)=1$ and the result is proven.

Corollary 8.3. Let $(K, D) \in \mathscr{L Q}$. Write $D(k)=\sum k^{(1)} \otimes \ldots \otimes k^{(p)} \in C_{q}\left(K^{P}\right)$ for $k \in C_{q}(K)$, and regard each $k^{(i)}$ as an element of $C_{q}(K)$. Let $x \in C^{q}(K)$ be a cocycle. Then $P^{o}\{x\}$ is represented by that cocycle $y \in C^{q}(K)$ such that $y(k)=\sum x\left(k^{(1)}\right) \cdots x\left(k^{(p)}\right) \in z_{p}$ for each $k \in C_{q}(K)$. In particular, if $D(k)=N \ell \in K^{p}$ for each $k \in K$, whe re $N=\sum \alpha^{i} \in Z_{p} \pi$, then $P^{0}=0$ on $H^{*}(K)$.

Proof. By formulas (5.1) and (5.2), $\left.y=\nu(-q) \theta(e) q(p-1) \otimes x^{p}\right)$ represents $\mathrm{P}^{\circ}\{\mathrm{x}\}$, and the $\mathrm{x}$ esult follows by an easy computation from Definition 7.5 and the lemma.

We now give a useful application of the theory for which the Steenrod operations satisfy the results of Theorem 7.9 and $P^{\circ}=0$. Let $\mathcal{L}$ and $y$ denote the categories of restricted Lie algebras and of primitively generated Hopf algebras 
over $Z_{\mathrm{p}}$. Let $\mathrm{F}: Q \rightarrow \mathscr{L}$ denote the free restricted Lie algebra functor, let $\mathrm{v}: \mathscr{L} \longrightarrow \mathcal{H}$ denote the universal enveloping algebra functor, and let $\mathrm{P}: \mathcal{H} \rightarrow \mathscr{K}$ denote the functor which assigns to $H \in \mathscr{A}$ its restricted Lie algebra of primitive elements. By a result of Milnor and Moore [19, Theorem 6.11], PV(L) = L for $L \in \mathscr{L}$ and $V P H=H$ for $H \in \mathscr{H}$. By Theorems of Witt and Friedrich $[9$, Theorems 7 and 9, p.168-170], extended to restricted Lie algebras, if $k \in a$ and $T(K)$ is the tensor algebra of $K$, then $V(F K)=T(K)$ in $\not$ and $F K=P T(K)$ in $\mathscr{L}$. These statements clearly remain valid for the categories $\& \mathcal{L}, \mathcal{L}$ and 1* of simplicial objects in $a, x$, and $\mathcal{H}$ [see 15, Definition 2.1]. We shall need the following algebraic lemma.

Lemma 8.4. Let $L$ be a restricted Lie algebra and let $I V(L)=\operatorname{Ker} \varepsilon$, $\varepsilon: V(L) \rightarrow Z_{p}$, be its augmentation ideal. Let $\psi: V(L) \longrightarrow V(L)^{P}=V(L) \otimes \ldots \otimes V(L)$ denote the iterated coproduct. Then, for each $x \in \operatorname{IV}(L)$, there exists $y \in V(L)^{p}$ such that $\psi(x)=N_{y}$.

Proof. Let $\pi: F K \rightarrow L$ represent $L$ as a quotient of a free restricted Lie algebra. Then $\mathrm{V}(\pi)=\mathrm{V}(\mathrm{FK}) \rightarrow \mathrm{V}(\mathrm{L})$ is an epimorphism of Hopf algebras, and we may assume that $L=F K$. Clearly we may also assume that $K$ is a finite dimensional $\mathrm{Z}_{\mathrm{p}}$-module. Since $\mathrm{T}(\mathrm{K})$ admits a grading under which it is connected, [19, Proposition 4.20] implies that the p-th power operation $\xi$ is zero on the augmentation ideal of the dual Hopf algebra $T(K)^{*}$. The cocommutativity of $T(K)$ implies that, for $x \in \operatorname{IT}(K), \psi(x)$ can be written in the form $\psi(x)=N y+\sum z_{i} \otimes \ldots \otimes z_{i}$ in $T(K)^{p}$. By the triviality of $\xi$ on $\operatorname{IT}(K)^{*}$, each $\mathbf{z}_{\mathrm{i}}=0$ and the result follows.

We now sketch a definitional framework for the study of homotopy invariants of simplicial restricted Lie algebras. Define a category $\mathscr{L W}_{2}$ as follows. The objects of $\delta \mathcal{X}_{2}$ are pairs $(L, M)$ such that $L \in \delta \mathscr{L}$ and $M$ is a restricted Lie ideal of $L$ and the morphisms $f:(L, M) \rightarrow\left(L^{\prime}, M^{\prime}\right)$ in $\& \mathscr{L}_{2}$ are morphisms 
$\mathrm{f}: L \rightarrow L^{\prime}$ in $\& \mathcal{L}$ such that $f(M) \subset M^{\prime}$. Two such morphisms, $f$ and $g$, are said to be Lie homotopic if there exist morphisms of restricted Lie algebras $h_{i}: L_{q} \rightarrow L_{q+1}^{\prime}, 0 \leq i \leq q$, such that $h_{i}\left(M_{q}\right) \subset M_{q+1}^{\prime}$ and the identities (i) - (iii) of [15, Definition 5.1] are satisfied. Define the homotopy, homology, and cohomology groups of $(\mathrm{L}, \mathrm{M}) \in \mathbb{\&} \mathscr{Z}_{2}$ by

$$
\begin{aligned}
& \pi_{* *}(L, M)=H_{*}(L / M) \text { and } \\
& H_{* *}(L, M)=H_{* *}(I V(L / M)) \text { and } H^{*}(L, M)=H^{*}(\operatorname{IV}(L / M))
\end{aligned}
$$

The homology and cohomology groups on the right sides of these equations are as defined at the start of section 7 , with $L / M$ and $I V(L / M)$ regarded as simplicial $\mathrm{Z}_{\mathrm{p}}$-modules. The argument of [15, Proposition 5.3] shows that Lie homotopic morphisms in $\& \mathscr{Z}_{2}$ induce the same morphisms on homotopy, homology, and cohomology. By [15, Theorem 22.1], $\pi_{*}(L, M)$ and $H_{*}(L, M)$ are, respectively, the homotopy groups of $L / M$ and of IV(L/M) regarded as simplicial sets. The Hurewicz homomorphism $h: \pi_{*}(L, M) \rightarrow \mathrm{H}_{*}(L, M)$ may thus be defined as the map induced on homotopy from the inclusion $L / M \rightarrow I V(L / M)$. Since $\operatorname{IV}(\mathrm{L} / \mathrm{M})=\operatorname{IV}(\mathrm{L}) / \mathrm{IV}(\mathrm{M})$, we have natural long exact homotopy, homology, and cohomology sequences on pairs $(L, M) \in \mathscr{L}_{2}$, and $h$ defines a natural transformation of long exact sequences. Note that $H_{*}(L, M)$ is the augmentation ideal of the Hopf algebra $\mathrm{H}_{*}(\mathrm{~V}(\mathrm{~L} / \mathrm{M}))$ if $\mathrm{H}_{*}(\mathrm{~V}(\mathrm{~L} / \mathrm{M}))$ is of finite type. Consider $F A S^{n}=F\left(\tilde{S}^{n} \otimes z_{p}\right)$, where $S^{n}$ is the simplicial $n$-sphere. It can be proven that $\pi_{n}(L)$ is the $Z_{p}$-module of Lie homotopy equivalence classes of morphisms $\mathrm{FAS}^{\mathrm{n}} \rightarrow L$ for $L \in \& \mathscr{L}$ and that $\mathrm{H}_{*}\left(\mathrm{FAS}^{\mathrm{n}}\right) \cong \tilde{\mathrm{H}}_{*}\left(\Omega \mathrm{S}^{\mathrm{n}+1}\right)$ is the augmentation ideal of the free commutative algebra on one primitive generator of degree $n$.

Our theory immediately yields Steenrod operations on $H^{*}(L, M)$.

Theorem 8.5. There exist natural homomorphisms $P^{s}$ and, if $p>2$, $\beta P^{S}$ defined on $H^{*}(L, M)$ for $(L, M) \in\left\langle\mathcal{G}_{2}\right.$. These operations satisfy the con- 
clusions of Theorem 7.9 (except that the hypothesis of (iii) is not satisfied in general) and, in addition, the operation $\mathrm{P}^{\circ}$ is identically zero.

Proof. We may regard $C_{*}(L, M)=C(I V(L / M))$ as an object of $10 a$, with diagonal $\bar{D}=\bar{\psi}$, the reduced coproduct as defined in Remarks 7.4(ii). Thus Theorem 7.9 applies directly, and $P^{\circ}=0$ follows from the previous lemma and corollary.

In [22], Priddy has given a different definition of $H_{*}(L)$ and $H^{*}(L)$. Let $\bar{W}$ be the functor from simplicial $Z_{p}$-algebras to $\& Q$ defined by Moore [20]. If $A$ is a simplicial $z_{p}$-algebra, then $\bar{W}_{o}(A)=Z_{p}$ and $\bar{W}_{q}(A)=A_{q-1} \otimes \ldots \otimes A_{0}$, $\mathrm{q}>0$, as $\mathrm{Z}_{\mathrm{p}}$-modules. The face and degeneracy operators are as defined in $[15$, p. 87]. For $L \in \not \mathcal{L}, \bar{W} V(L)$ is a simplicial cocommutative coalgebra with coproduct $\psi$ given by

$$
\psi\left(a_{q-1} \otimes \ldots \otimes a_{0}\right)=\sum\left(a_{q-1}^{\prime} \otimes \ldots \otimes a_{0}^{\prime}\right) \otimes\left(a_{a-1}^{n} \otimes \ldots \otimes a_{0}^{\prime}\right)
$$

where $a_{i} \in V_{i}(L)$ satisfies $\psi\left(a_{i}\right)=\sum a_{i}^{\prime} \otimes a_{i}^{\prime \prime}$. Priddy defines $H_{*}(L)=H_{*}(I \bar{W} V(L))$ and $H^{*}(L)=H^{*}(I \bar{W} V(L))$, where $I \bar{W} V(L)$ is regarded as a simplicial $\mathrm{z}_{\mathrm{p}}$-module. For spectra, Priddy's definition and ours clearly differ only by a shift of degree; with his definition, $H^{*}\left(F A S^{n}\right) \cong \tilde{H}^{*}\left(S^{n+1}\right)=z_{p}$. By Definition 7.3 and Remarks $7.4(\mathrm{ii}),(\mathrm{C}(\mathrm{I} \overline{\mathrm{W}} \mathrm{V}(\mathrm{L}), \bar{\psi}) \in \mathbb{1} Q \mathrm{a}$ and the refore Priddy's $H^{* *}(L)$ alos admits Steenrod operations which satisfy the conclusions of Theorem 7.9 (except, in general, for (iii)) and $\mathrm{P}^{\circ}=0$.

\section{The dual homology operations; Nishida's theorem}

For applications to loop spaces and to obtain a result used in the proof of the Adem relations, we shall discuss the homology operations $P_{*}^{S}$ whose duals are the Steenrod operations on the mod $p$ cohomology of a space $X$. Of course, $H^{*}(X)=H_{*}(X)^{*}=\operatorname{Hom}_{Z_{p}}\left(H_{*}(X), Z_{p}\right)$ and, if $H_{*}(X)$ is of finite type, $H_{*}(X)=H^{*}(X)$. Define $P_{*}^{s}$ on $H_{*}(X)$ by $P^{s}=\left(P_{*}^{s}\right)^{*} ; P_{*}^{s}$ is clearly well-defined if $H_{*}(X)$ is of 
finite type, and either a direct limit argument or the next proposition imply that $P_{*}^{s}$ is well-defined in general. $P_{*}^{s}$ lowers degrees by $s$ if $p=2$ and by $2 s(p-1)$ if $p>2$. Our results on the $P^{S}$ immediately yield the dual results for the $P_{*}^{S}$. We shall write the operations $P_{*}^{s}$ on the left; the order of composition in the dual Adem relations must thus be reversed (that is, $H_{*}(X)$ is a left module over the opposite algebra of the Steenrod algebra). The following proposition was used in the proof of Lemma 4.6. Formula (2) of the proof is essentially Steenrod's definition $[30]$ of the $D_{i}$.

Proposition 9.1. Let $\mathrm{X}$ be a space and let $\mathrm{d}=\Phi(1 \otimes \mathrm{D}): \mathrm{W} \otimes_{\pi} \mathrm{C}_{*}(\mathrm{X}) \rightarrow$ $W \otimes_{\pi} C_{*}(X)^{P}$. Consider $d_{*}: H_{* k}\left(\pi ; H_{*}(X)\right) \rightarrow H_{*}\left(\pi ; H_{*}(X)^{P}\right)$. Let $x \in H_{s}(X)$. Then (i) If $\mathrm{p}=2, \mathrm{~d}_{*}\left(\mathrm{e}_{\mathrm{r}} \otimes \mathrm{x}\right)=\sum_{\mathrm{k}} \mathrm{e}_{\mathrm{r}+2 \mathrm{k}-\mathrm{s}} \otimes \mathrm{P}_{*}^{\mathrm{k}}(\mathrm{x}) \otimes \mathrm{P}_{*}^{\mathrm{k}}(\mathrm{x})$.

(ii) If $\mathrm{p}>2, \quad \mathrm{~d}_{*}\left(\mathrm{e}_{\mathrm{r}} \otimes \mathrm{x}\right)=v(\mathrm{~s}) \sum_{\mathrm{k}}(-1)^{\mathrm{k}} \mathrm{e}_{\mathrm{r}+(2 \mathrm{pk}-\mathrm{s})(\mathrm{p}-1)} \otimes \mathrm{P}_{*}^{\mathrm{k}}(\mathrm{x})^{\mathrm{p}}$

$$
\text { - } \delta(r) v(s-1) \sum_{k}(-1)^{k} e_{r+p+(2 p k-s)(p-1)} \otimes P_{*}^{k} \beta(x)^{p} \text {. }
$$

where $\nu(2 \mathbf{j}+\varepsilon)=(-1)^{\mathbf{j}}(\mathrm{m} !)^{\boldsymbol{\varepsilon}}$ and $\delta(2 j+\varepsilon)=\boldsymbol{\varepsilon}, \quad \varepsilon=0$ or 1 .

Proof. We may assume that $\mathrm{H}_{n^{*}}(\mathrm{X})$ is of finite type. We shall compute $d^{*}: H^{*}\left(\pi ; H^{*}(X)^{p}\right) \rightarrow H^{*}(\pi) \otimes H^{*}(X)$ and then dualize. In the notations of Lemma 1.3, $H_{*}(X)^{\mathrm{P}} \cong \mathrm{A} \oplus \mathrm{Z}_{\mathrm{p}} \pi \otimes \mathrm{B}$ as a $\pi$-module, and $H_{*}\left(\pi ; H_{*}(X)^{p}\right) \cong H_{*}(\pi) \otimes A \oplus B$. It follows that $H^{*}\left(\pi ; H^{*}(X)^{p}\right) \cong H^{*}(\pi) \otimes A^{*} \oplus B^{*}$. We claim first that $d^{*}\left(B^{*}\right)=0$. To see this, we make explicit the isomorphism from $B^{*}$ to the homology of $\left(W \otimes_{\pi} Z_{p} \pi B\right)^{*}$. For $y \in B^{*}$, define $\tilde{y} \in\left(W \otimes_{\pi} z_{p} \pi \otimes B\right)^{* *}$ by

$$
\tilde{y}\left(w \otimes \alpha^{i} \otimes b\right)=\varepsilon(w) y(b) \text { for } w \in W, 0 \leq i<p, \quad b \in B .
$$

Then $y$ is a cocylce and $y \rightarrow \tilde{y}$ induces the desired isomorphism. Define $\nu: \mathrm{Z}_{\mathrm{p}} \pi \rightarrow \mathrm{Z}_{\mathrm{p}}$ by $\nu(1)=1$ and $\nu\left(\alpha^{i}\right)=0,1 \leq \mathrm{i}<\mathrm{p}$, and define $\bar{y} \in\left(\mathrm{W} \otimes \mathrm{Z}_{\mathrm{p}} \pi \otimes \mathrm{B}\right) *$ for $y \in B^{*}$ by

$$
\bar{y}\left(w \otimes \alpha^{i} \otimes b\right)=\varepsilon(w) \nu\left(\alpha^{i}\right) y(b) \quad \text { for } w \in W, 0 \leq i<p, b \in B
$$


Clearly $\tilde{\mathrm{y}}\left(\mathrm{w} \otimes \alpha^{i} \otimes \mathrm{b}\right)=\overline{\mathrm{y}}\left(\mathrm{N}\left[\mathrm{w} \otimes \alpha^{i} \otimes \mathrm{b}\right]\right)$. Therefore

$$
\mathrm{d}^{*}(\tilde{y})(w \otimes x)=\tilde{y} d_{\pi^{*}}(w \otimes x)=\bar{y} d_{*_{k}}(N w \otimes x)=0
$$

for $w \in W$ and $x \in H_{*}(X)$ since $N w \otimes x$ is a boundary in $W \otimes H_{*}(X)$ (because $d\left(e_{2 i}\right)=N e_{2 i-1}$ and $d\left(T^{p-2} e_{2 i+1}\right)=N e_{2 i}$ in $\left.W\right)$. This proves that $d^{*}\left(B^{*}\right)=0$. We next compute $d^{*}$ on $H^{* *}(\pi) \otimes A^{*}$. Let $y \in H^{q}(x)$ and $x \in H_{p q-i}(x)$. By Definition 7.5 , we have the formula

$$
D_{i}(y)(x)=\theta_{*}\left(e_{i} \otimes y^{p}\right)(x)=(-1)^{i q_{d}^{*}}(\varepsilon \otimes 1)^{*}\left(y^{p}\right)\left(e_{i} \otimes x\right)
$$

(where the isomorphism $\alpha$ from the tensor product of duals to the dual of tensor products has been omitted from the notation).

Let $w_{i}$ be dual to $e_{i}$. Then $(\varepsilon \otimes 1)^{*}\left(y^{p}\right)=w_{0} \otimes y^{p}$ and therefore

$$
d^{* *}\left(w_{0} \otimes y^{p}\right)\left(e_{i} \otimes x\right)=(-1)^{i q_{D_{i}}}(y)(x)
$$

For any $z \in H^{p q-i}(X)$, the sign in the definition of $\alpha$ gives

$$
\left(w_{i} \otimes z\right)\left(e_{i} \otimes x\right)=(-1)^{i(p q-i)} z(x) \text {. }
$$

Comparing these formulas, we see that

$$
\mathrm{d}^{*}\left(\mathrm{w}_{\mathrm{o}} \otimes \mathrm{y}^{\mathrm{P}}\right)=\sum(-1)^{\mathrm{i}} \mathrm{w}_{\mathrm{i}} \otimes \mathrm{D}_{\mathrm{i}}(\mathrm{y})
$$

To compute $d^{*}\left(w_{j} \otimes y^{p}\right)$ for $j>0$, observe that if $\rho: W \rightarrow \bar{W}=Z_{p} \otimes_{\pi} W$ is the natural epimorphism, then we have the commutative diagram:

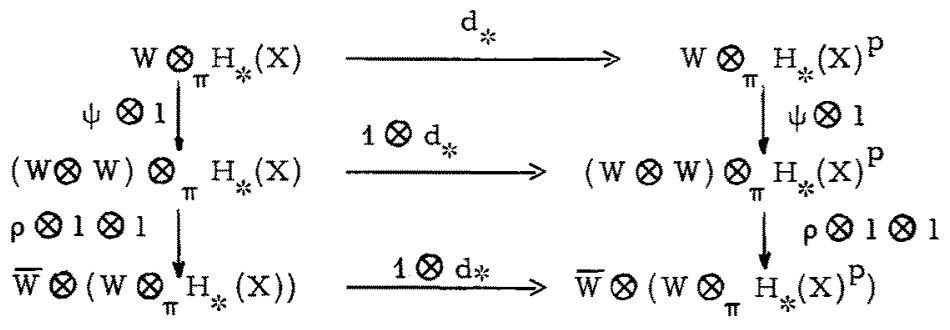

(The upper rectangle requires an easy acyclic models argument.) Dually, $d^{*}$ is a morphism of $H^{*}(\pi)$-modules. Now $w_{j}\left(w_{0} \otimes y^{p}\right)=w_{j} \otimes y^{p}$ and, in $H^{*}(\pi)$, $w_{j} w_{i}=w_{i+j}$ if $p=2$ or either $i$ or $j$ is even and $w_{j}=0$ if $p>2$ and $i$ and $j$ are odd by formula $(1,2)$. Therefore (2) implies the formulas 
(3) if $\mathrm{p}=2, \mathrm{~d}^{*}\left(\mathrm{w}_{\mathrm{j}} \otimes \mathrm{y}^{\mathrm{p}}\right)=\sum_{\mathrm{i}} \mathrm{w}_{\mathrm{i}+\mathrm{j}} \otimes \mathrm{D}_{\mathrm{i}}(\mathrm{y}) \quad$ and

(4) if $p>2, \quad d^{*}\left(w_{j} \otimes y^{p}\right)=\sum_{i} w_{2 i+j} \otimes D_{2 i}(y)-\delta(j+1) \sum w_{2 i+1+j} \otimes D_{2 i+1}(y)$.

By formulas (5.1) and (5.2) and a reindexing, (3) and (4) become

(5) if $p=2, \quad d^{*}\left(w_{j} \otimes y^{p}\right)=\sum_{k} w_{j+q-k} \otimes P^{k}(y) \quad$ and

(6) if $p>2, \quad d^{*}\left(w_{j} \otimes y^{p}\right)=v(-q)^{-1} \sum_{k}(-1)^{k} w_{j}+(q-2 k)(p-1)^{\otimes P^{k}(y)}$

$$
-\delta(j+1) v(-q)^{-1} \sum_{k}(-1)^{k} w_{j+(q-2 k)}(p-1)-1 \otimes \beta P^{k}(y) \text {. }
$$

We now dualize. $d_{*}\left(H_{*}(\pi) \otimes H_{*}(X)\right) \subset H_{*}(\pi) \otimes A$ since $d^{*}\left(B^{*}\right)=0$. For $x \in H_{s}(X)$, we may therefore write

$$
\mathrm{d}_{*}\left(\mathrm{e}_{\mathrm{r}} \otimes \mathrm{x}\right)=\sum \mathrm{e}_{\mathrm{r}+\mathrm{s}-\mathrm{pq}} \otimes E_{\mathrm{qr}}(\mathrm{x})^{\mathrm{p}}, \mathrm{E}_{\mathrm{qr}}(\mathrm{x}) \in \mathrm{H}_{\mathrm{q}}(\mathrm{x}) .
$$

Let $y \in H^{q}(X)$. Using the Kronecker pairing $<$, $>$, we have

(7) $\quad\left\langle w_{r+s-p q} \otimes y^{p}, d_{*}\left(e_{r} \otimes x\right)>=(-1)^{(r+s-q+m) q}<y, E_{q r}(x)>\right.$.

Since $\left\langle\mathrm{P}^{\mathrm{k}}(\mathrm{y}), \mathrm{x}\right\rangle=\left\langle\mathrm{y}, \mathrm{P}_{*}^{\mathrm{k}}(\mathrm{x})\right\rangle$, (5) implies that if $\mathrm{P}=2$, then

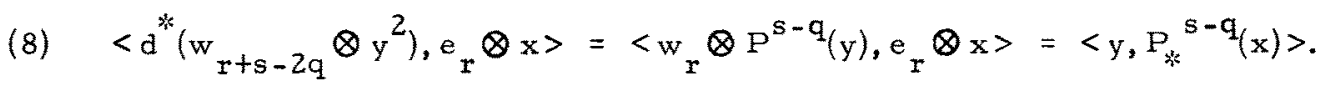

Thus $E_{q r}(x)=P_{*}^{s-q}(x)$ if $p=2$ and, with $k=s-q$, this implies (i). Now assume that $\mathrm{p}>2$. By $(6), \mathrm{d}^{*}\left(\mathrm{w}_{\mathrm{r}+\mathrm{s}-\mathrm{pq}} \otimes \mathrm{y}^{\mathrm{p}}\right)$ has a summand involving $\mathrm{w}_{\mathrm{r}}$ only if $q=s-2 k(p-1)-\varepsilon, k \geq 0$ and $\varepsilon=0$ or 1 , hence $E_{q r}(x)=0$ for other values of $q$.

For $q=s-2 k(p-1),(6)$ gives

(9) $<\mathrm{d}^{*}\left(\mathrm{w}_{\mathrm{r}+(2 \mathrm{pk}-\mathrm{s})(\mathrm{p}-1)} \otimes \mathrm{y}^{\mathrm{p}}\right), \mathrm{e}_{\mathrm{I}} \otimes \mathrm{x}>=v(-\mathrm{q})^{-1}(-1)^{\mathrm{k}+\mathrm{rq}}<\mathrm{y}, \mathrm{P}_{*}^{\mathrm{k}}(\mathrm{x})>$.

By (7) and (9), $E_{q x}(x)=(-1)^{k+m q} v(-q)^{-1} P_{*}^{k}(x)$ if $q=s-2 k(p-1)$; since $(-1)^{\mathrm{mq}} v(-\mathrm{q})^{-1}=v(\mathrm{q})=v(\mathrm{~s})$, this yields the first sum of (ii). Observe next that $\left.\langle\beta y, x\rangle=(-1)^{q+1}<y, \beta x\right\rangle$ by the chain and cochain definitions of the Bockstein and the sign convention $\delta(f)=(-1)^{\text {deg } f+1}$ fd used in defining $C^{*}(X)$. Now for $q=s-2 k(p-1)-1,(6)$ gives 
(10) $\left\langle\mathrm{d}^{*}\left(w_{\mathrm{r}+\mathrm{p}+(2 \mathrm{pk}-\mathrm{s})(\mathrm{p}-1)} \otimes \mathrm{y}^{\mathrm{p}}\right), \mathrm{e}_{\mathrm{r}} \otimes \mathrm{x}>=\delta ! \mathrm{r}\right) \nu(-q)^{-1}(-1)^{\mathrm{k}+\mathrm{r}(\mathrm{q}+1)+q}<\mathrm{y}, \mathrm{P}_{*}^{\mathrm{k}} \beta(\mathrm{x})>$ By (7) and (10), $E_{q r}(x)=(-1)^{k+r+m q}{ }_{\nu}(-q)^{-1} \delta(r) P_{*}^{k} \beta(x)$ if $q=s-2 k(p-1)-1 ;$ since $\delta(r)=0$ if $r$ is even and $(-1)^{m q} v(-q)^{-1}=v(q)=v(s-1)$, this yields the second sum of (ii) and so completes the proof.

Remark 9.2. The proof above used no properties specific to topological spaces and so applies to compute

$$
d_{*}=\Phi_{*}(1 \otimes D)_{*}: H_{*}\left(\pi ; H_{*}(K)\right) \rightarrow H_{*}\left(\pi ; H_{*}(K)^{p}\right)
$$

in terms of $\mathrm{P}_{*}^{\mathrm{s}}$ and $\beta \mathrm{P}_{*}^{\mathrm{s}}$ (where $\beta \mathrm{P}_{*}^{\mathrm{s}}$ is defined by $\left(\beta \mathrm{P}_{*}^{\mathrm{s}}\right)^{*}=-\beta \mathrm{P}^{\mathrm{s}}$ if no Bockstein is present) for arbitrary objects $(K, D) \in D Q$.

We now give a new proof of a result due to Nishida [21], which is essential to the computation of Steenrod operations in iterated loop spaces. Let $\mathrm{K}\left(\mathrm{Z}_{\mathrm{p}}, 1\right)=\mathrm{E} / \pi$ where $\pi$ operates properly on the acyclic space $E ;$ by [14, IV 11], $C_{*}(E)=Z_{p} \pi \otimes C_{*}(E / \pi)$. Let $\sigma: E \rightarrow E / \pi$ be the projection and let $f: W \rightarrow C_{*}(E)$ be a $\pi$-morphism over $Z_{p}$. If $\bar{W}=Z_{p} \otimes_{\pi} W$, then $f$ induces $\bar{f}: \bar{W} \rightarrow C_{*}(E / \pi)$, and $\bar{f}_{\rho}$ is homotopic to $\sigma f, \rho: W \rightarrow \bar{W}$. By Remarks 7.2, if $\eta$ is the shuffle map, then we have the following homotopy commutative diagram for any space $\mathrm{X}$ :

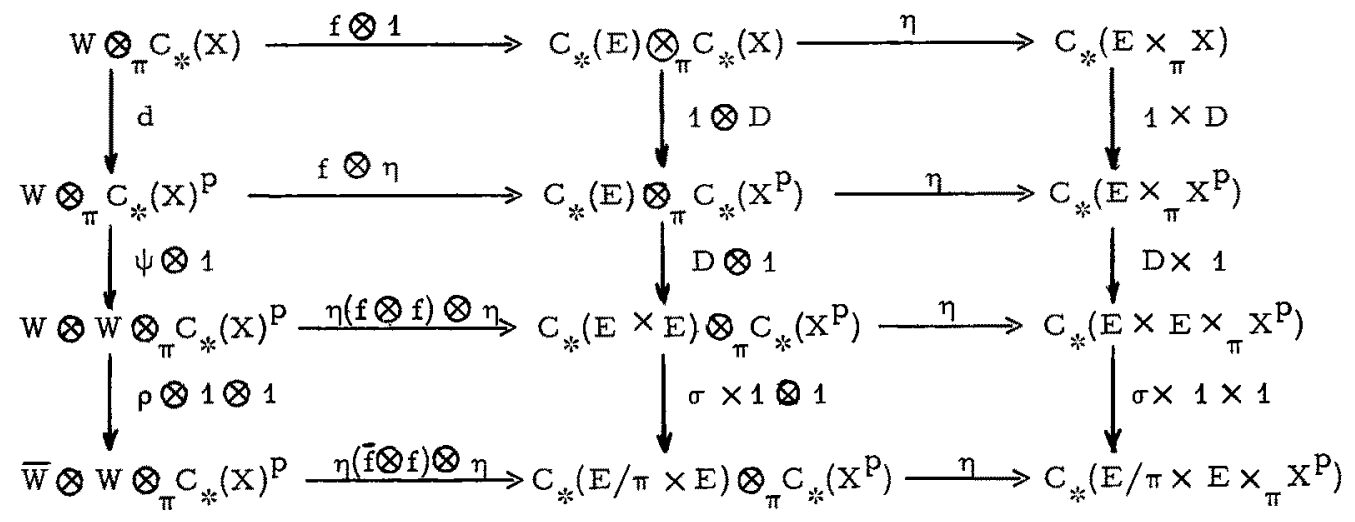

Let $\mu_{*}=(\rho \otimes 1 \otimes 1)_{*}(\psi \otimes 1)_{*}: H_{*}\left(\pi ; H_{*}(X)^{\mathrm{P}}\right) \longrightarrow \mathrm{H}_{*}(\pi) \otimes \mathrm{H}_{*}\left(\pi ; H_{*}(X)^{\mathrm{p}}\right)$. The horizontal arrows are homology isomorphisms and we therefore have Steenrod operations $\mathrm{P}_{*}^{\mathrm{s}}$ on $\mathrm{H}_{*}\left(\pi ; \mathrm{H}_{*}(\mathrm{X})\right), \mathrm{H}_{*}\left(\pi ; \mathrm{H}_{*}(\mathrm{X})^{\mathrm{P}}\right)$, and $\mathrm{H}_{*}(\pi) \otimes \mathrm{H}_{*}\left(\pi ; \mathrm{H}_{*}(\mathrm{X})^{\mathrm{P}}\right)$ such that $\mathrm{d}_{*}$ 
and $\mu_{*}$ commute with the $P_{*}^{S}$. The following theorem uses $d_{*}$ and $\mu_{*}$ to evaluate the $\mathrm{P}_{*}^{\mathrm{s}}$ on $\mathrm{H}_{*}\left(\pi ; \mathrm{H}_{*}(\mathrm{X})^{\mathrm{P}}\right)$. Our result differs from Nishida's by a sign; the reason for this is that our formulas (2) and (6) in the proof above differ from the corresponding formulas in [30,p.103 and p.119]. We were pedantic about signs in the preceding proof because of this disagreement. We shall need the following identity on binomial coefficients in the proof of the theorem.

Lemma 9.3. $\sum_{i}(i, a-i)(n-i, i+b-n)=(n, a+b-n)$ for $a \geq 0, b \geq 0$, and $n \geq 0$.

Proof. The result is obvious if $b=0$, when $i=n$ gives the only non-zero summand on the left. Using $(c-1, d)+(c, d-1)=(c, d)$, we find that the result for the triples $(a, b-1, n)$ and $(a, b-1, n-1)$ implies the result for the triple $(a, b, n)$.

Theorem 9.4. Let $X$ be a space, $x \in \mathrm{H}_{\mathrm{q}}(\mathrm{X})$. Then, in $\mathrm{H}_{*}\left(\pi ; \mathrm{H}_{*}(\mathrm{X})^{\mathrm{P}}\right)$, (i) If $p=2, \quad P_{*}^{s}\left(e_{r} \otimes x^{2}\right)=\sum_{i}(s-2 i, r+q-2 s+2 i) e_{r-s+2 i} \otimes P_{*}^{i}(x)^{2}$. (ii) If $p>2, P_{*}^{s}\left(e_{r} \otimes x^{p}\right)=\sum\left(s-p i,\left[\frac{r}{2}\right]+q m-p s+p i\right) e_{r+2(p i-s)(p-1)} \otimes P_{*}^{i}(x)^{p}$ $+\delta(\mathbf{r}) \alpha(q) \sum_{i}\left(s-p i-1,\left[\frac{r+1}{2}\right]+q m-p s+p i\right) e_{r+p+2(p i-s)(p-1)} \otimes P_{*}^{i} \beta(x)^{p}$ where $\alpha(q)=\nu(q)^{-1} \nu(q-1)=-(-1)^{m q} m !$ and $\delta(2 j+\varepsilon)=\varepsilon, \varepsilon=0$ or 1 .

Proof. We assume that $s>0$, since the result is trivial for $s=0$. If $r=0$, then $e_{0} \otimes x^{p}$ is in the image of $H_{*}\left(E \times X^{p}\right) \rightarrow H_{*}\left(E \times x^{p}\right)$. In $H_{*}\left(E \times X^{p}\right)$, $P_{*}^{s}\left(e_{0} \otimes x^{p}\right)=\sum e_{0} \otimes P_{*}^{i_{1}}(x) \otimes \ldots \otimes P_{*}^{i} p^{i}(x)$ summed over all p-tuples $\left(i_{1}, \ldots, i_{p}\right)$ such that $\sum i_{p}=s$. The sum of all terms with any $i_{j} \neq i_{k}$ lies in $e_{0} \otimes N H_{*}(x)^{p}$ and is thus zero in $\mathrm{H}_{*}\left(\mathrm{E} \times \times_{\pi} \mathrm{X}^{\mathrm{P}}\right)$. Therefore $\mathrm{P}_{*}^{\mathrm{s}}\left(\mathrm{e}_{0} \otimes \mathrm{x}^{\mathrm{P}}\right)=0$ unless $\mathrm{s}=\mathrm{pt}$, when $\mathrm{P}_{*}^{\mathrm{s}}\left(\mathrm{e}_{0} \otimes \mathrm{x}^{\mathrm{p}}\right)=\mathrm{e}_{0} \otimes \mathrm{P}_{*}^{\mathrm{t}}(\mathrm{x})^{\mathrm{p}}$, which is in agreement with (i) and (ii). Recall that, by Definition 1.2 and the proof of Lemma 4.6, we have the following relations in $\mathrm{H}_{*}(\pi)$.

(a) If $p=2, P_{*}{ }^{i}\left(e_{j}\right)=(i, j-2 i) e_{j-i}$ and $\psi\left(e_{r}\right)=\sum e_{j} \otimes e_{r-j}$ 
(b) If $p>2, P_{*}^{i}\left(e_{j}\right)=(i,[j / 2]-p i) e_{j-2 i(p-1)}$ and $\psi\left(e_{r}\right)=\sum \delta(r, j) e_{j} \otimes e_{r-j}$ where $\delta(r, j)=1$ unless $r$ is even and $j$ is odd, when $\delta(r, j)=0$. If $q=0$, then $d_{*}\left(e_{r} \otimes x\right)=e_{r} \otimes x^{p}$ and therefore

$$
P_{*}^{s}\left(e_{r} \otimes x^{p}\right)=d_{*}\left(P_{*}^{s}\left(e_{r}\right) \otimes x\right)=P_{*}^{s}\left(e_{r}\right) \otimes x^{p} ;
$$

by (a) and (b), the result holds in this case. We now proceed by induction on $q$ and for fixed $q$ by induction on $r$. Thus assume the result for $q^{1}<q$ and for our fixed $q$ and $r^{\prime}<r$. Let $z=P_{*}^{S}\left(e_{r} \otimes x\right)$ and let $z^{\prime}$ denote the right side of the equation to be proven. Write $z-z^{\prime}=\sum e_{i} \otimes y_{i} \in H_{*}\left(\pi ; H_{*}(X)^{p}\right)$. We shall first prove that $\mu_{*}\left(z-z^{\prime}\right)=e_{0} \otimes\left(z-z^{\prime}\right)$; this will imply that $y_{i}=0$ for all $i>0$ since if $i$ is maximal such that $y_{i} \neq 0$, then $e_{i} \otimes e_{0} \otimes y_{i}$ clearly occurs as a non-zero summand of $\mu_{* k}\left(z-z^{\prime}\right)$. We shall then prove that $y_{0}=0$ by explicit computation and so complete the proof. We give the details separately in the cases $p=2$ and $p>2$.

(i) $p=2$. Since $\mu_{*}(z)=P_{*}^{s} \mu_{*}\left(e_{x} \otimes x^{2}\right)$, we find by $(a)$, the Cartan formula in $\mathrm{H}_{*}(\pi) \otimes \mathrm{H}_{*}\left(\pi ; \mathrm{H}_{*}(\mathrm{X})^{2}\right)$, and the induction hypothesis on $r$, that

(c) $\quad \mu_{*}(z)=P_{*}^{s}\left(\sum_{j} e_{j} \otimes e_{r-j} \otimes x^{2}\right)=\sum_{i, j}(i, j-2 i) e_{j-i} \otimes P_{*}^{s-i}\left(e_{r-j} \otimes x^{2}\right)$, where

$$
P_{*}^{s-i}\left(e_{r-j} \otimes x^{2}\right)=\sum_{k}(s-i-2 k, r-j+q-2 s+2 i+2 k) e_{r-j-s+i+2 k} \otimes P_{*}^{k}(x)^{2} \text { if } j>0 .
$$

The terms with $j=i>0$ are zero since $(i,-i)=0$. Applying the lemma to those $(i, j)$ such that $j-i=\ell>0$, with $a=\ell, b=r-\ell+q-s$, and $n=s-2 k$, we see that (c) reduces to the formula

(d) $\quad \mu_{* k}(z)=e_{0} \otimes z+\sum_{k, \ell>0}(s-2 k, r+q-2 s+2 k) e_{\ell} \otimes e_{r-s+2 k-\ell} \otimes P_{*}^{k}(x)^{2}$.

A glance at the right side of (i) shows that $\mu_{*}\left(z-z^{\prime}\right)=e_{0} \otimes\left(z-z^{\prime}\right)$, hence $y_{i}=0$ for i $>0$. To compute $y_{0}$, observe that $P^{\circ}=1$ and Proposition $9.1 \mathrm{imply}$

(e) $\quad e_{r} \otimes x^{2}=d_{* k}\left(e_{r+q} \otimes x\right)+\sum_{k>0} e_{r+2 k} \otimes P_{*}^{k}(x)^{2}$. 
$P_{*}^{s} d_{*}=d_{*} P_{*}^{s}$, the Cartan formula on $H_{*}(\pi) \otimes H_{*}(X)$, and Proposition 9.1 evaluate $P_{*}^{s} d_{*}\left(e_{r+q} \otimes x\right)$, and the induction hypothesis on $q$ evaluates $\mathrm{P}_{*}^{\mathrm{s}}\left(\mathrm{e}_{\mathrm{r}+2 \mathrm{k}} \otimes \mathrm{P}_{*}^{\mathrm{k}}(\mathrm{x})^{2}\right)$ for $\mathrm{k}>0$. Carrying out these computations, we find that (e) implies the formula

$$
\text { (f) } \quad \begin{aligned}
z= & \sum_{\mathrm{k}, \ell}(s-\ell, r+q-2 s+2 \ell) e_{r-s+2 k+2 \ell} \otimes P_{*}^{k} P_{*}^{\ell}(x)^{2} \\
& +\sum_{k>0, \ell}(s-2 \ell, r+q+k-2 s+2 \ell) e_{r-s+2 k+2 \ell} \otimes P_{*}^{\ell} P_{* ;}^{k}(x)^{2} .
\end{aligned}
$$

In principle, (f) must imply (i) directly, but our argument with $\mu_{*}$ shows that we need only consider those terms involving $e_{0}$, with $2(k+l)=s-r$. Let $t=k+l-s$ and $c=q-k-\ell ;$ then these terms become

(g) $\sum_{k \geq 0}(k-t, t+c-2 k) e_{0} \otimes P_{*}^{k} P_{*}^{s+t-k}(x)^{2}+\sum_{\ell<s+t}(c+l-s, s-2 l) e_{0} \otimes P_{*}^{\ell} P_{*}^{s+t-l}(x)^{2}$.

By formula (f) of the proof of Theorem 4.7, rephrased as in section 5 and dualized (with the order of composition reversed under dualization since we are writing the operations $P_{*}^{s}$ on the left), and by Remarks 4.8 , (g) would be zero if $l=s+t$ were allowed in the second sum; thus $(\mathrm{g})$ reduces to

$$
(c+t,-s-2 t) e_{0} \otimes P_{*}^{s+t}(x)^{2}=(q-s, r) e_{0} \otimes P_{*}^{1 / 2(s-r)}(x)^{2} .
$$

Since $(h)$ is equal to the summand of $z^{\prime}$ involving $e_{0}$, it follows that $y_{0}=0$.

(ii) p > 2. For brevity of notation, write $d=2(p-1)$. As in the case $p=2$, we find by (b) and induction on $r$ that

$$
\text { (i) } \begin{aligned}
& \mu_{* k}(z)=\sum_{i j} \delta(r, j)(i,[j / 2]-p i) e_{j-d i} \otimes P_{*}^{s-i}\left(e_{r-j} \otimes x^{p}\right) \text {, where, if } j>0, \\
& P_{*}^{s-i}\left(e_{r-j} \otimes x^{p}\right)=\sum_{k}\left(s-i-p k,\left[\frac{r-j}{2}\right]+q m-p s+p i+p k\right) e_{r-j+d(p k-s+i)} \otimes P_{*}^{k}(x)^{p} \\
& +\delta(r-j) \alpha(q) \sum_{k}\left(s-i-p k-1,\left[\frac{r-j+1}{2}\right]+q m-p s+p i+p k\right) e_{r-j+p+d(p k-s+i)} \otimes P_{*}^{k} \beta(x)^{p}
\end{aligned}
$$

The terms with $j=d i>0$ are zero. By the lemma, applied to those $(i, j)$ such that $j-d i=\ell>0$, with $a=[\ell / 2], b=\left[\frac{r-\ell}{2}\right]+q m-s(p-1)$, and $n=s-p k$ for the 
first sum and $a=[\ell / 2], b=\left[\frac{r-\ell-1}{2}\right]+q m-s(p-1)$, and $n=s-p k-1$ for the second sum, (i) reduces to

(j) $\quad \mu_{*}(z)=e_{0} \otimes z+\sum_{k, \ell>0} \delta(r, \ell)\left(s-p k,\left[\frac{r}{2}\right]+q m-p s+p k\right) e_{\ell} \otimes e_{r+d(p k-s)-\ell} \otimes P_{* k}^{k}(x)^{p}$

$+\sum_{k, \ell>0} \delta(r, \ell) \delta(r-\ell) \alpha(q)\left(s-p k-1,\left[\frac{r+1}{2}\right]+q m-p s+p k\right) e_{\ell} \otimes e_{r+p+d(p k-s)-\ell} \otimes P_{*}^{k} \beta(x)^{p}$.

Now $\delta(\mathbf{r}, \ell) \delta(\mathbf{r}-\ell)=\delta(\mathbf{r}) \delta(\mathbf{r}+1, \ell)$, and it follows from a glance at (ii) that $\mu_{*}\left(z-z^{\prime}\right)=e_{0} \otimes\left(z-z^{\prime}\right)$. Thus $y_{i}=0$ for $i>0$. To compute $y_{0}$, observe that Proposition 9.1 implies that

$$
\begin{aligned}
& e_{r} \otimes x^{p}=v(q)^{-1} d_{*}\left(e_{r+q(p-1)} \otimes x\right)-\sum_{k>0}(-1)^{k} e_{r+d p k} \otimes P_{* k}^{k}(x)^{p} \\
&+\delta(r) \alpha(q) \sum_{k}(-1)^{k} e_{r+p+d p k} \otimes P_{*}^{k} \beta(x) \\
& p
\end{aligned}
$$

Precisely as in the case $p=2$, we can compute $\mathrm{P}_{*}^{\mathrm{s}}$ on the right side of $(k)$; carrying out this computation, we find

(l) $\quad z=\sum_{k, \ell}(-1)^{k}(s-\ell,[r / 2]+q m-p s+p \ell) e_{r+d(p k+p \ell-s)} \otimes P_{*}^{k} P_{*}^{\ell}(x)^{p}$

$-\delta(r) \alpha(q) \sum_{k, \ell}(-1)^{k}(s-\ell,[r / 2]+q m-p s+p l) e_{r+p+d(p k+p l-s)} \otimes P_{*}^{k} \beta P_{*}^{\ell}(x)^{p}$

$-\sum_{k>0, \ell}(-1)^{k}(s-p \ell,[r / 2]+k(p-1)+q m-p s+p l) e{ }_{r+d(p k+p l-s)} \otimes P_{*}^{\ell} P_{*}^{k}(x)^{p}$

$-\delta(r) \alpha(q) \sum_{k>0, \ell}(-1)^{k}\left(s-p \ell-1,\left[\frac{r+1}{2}\right]+k(p-1)+q m-p s+p \ell\right) e_{r+p+d(p k+p l-s)} \otimes P_{*}^{\ell} \beta P_{*}^{k}(x)^{p}$

$+\delta(r) \alpha(q) \sum_{k, \ell}(-1)^{k}\left(s-p l,\left[\frac{r+1}{2}\right]+k(p-1)+q m-p s+p l\right) e_{r+p+d(p k+p l-s)} \otimes P_{*}^{\ell} P_{*}^{k} \beta(x)^{p}$.

Consider the first and third sums, with $r+d(p k+p \ell-s)=0$. Let $t=k+\ell-s$ and $c=q-d(k+l)$. Then these two sums become

(m) $\quad \sum_{k}(-1)^{k}(k-t, t+m c-p k) e_{0} \otimes P_{*}^{k} P_{*}^{s+t-k}(x)^{p}$

$$
-\sum_{\ell<s+t}(-1)^{s+t+l}(\ell+m c-s, s-p l) e_{0} \otimes P_{*}^{l} P_{*}^{s+t-l}(x)^{p}
$$

Consider the remaining sums of $(\ell)$, with $r+p+d(p k+p \ell-s)=0 ; \quad r$ is odd, hence $\delta(r)=1$. Let $t$ be as above and let $c^{\prime}=c-1$. Then these three sums become 
(n)

$$
\begin{aligned}
-\alpha(q) & \sum_{k}(-1)^{k}\left(k-t, t+m c^{\prime}-p k-1\right) e_{0} \otimes P_{*}^{k} \beta P_{*}^{\ell}(x)^{p} \\
& -\alpha(q) \sum_{\ell<s+t}(-1)^{s+t+l}\left(\ell+m c^{\prime}-s, s-p \ell-1\right) e_{0} \otimes P_{*}^{\ell} \beta P_{*}^{s+t-l}(x)^{p} \\
& +\alpha(q) \sum_{\ell}(-1)^{s+t+l}\left(\ell+m c^{\prime}-s, s-p l\right) e_{0} \otimes P_{*}^{\ell} P_{*}^{s+t-l} \beta(x)^{p} .
\end{aligned}
$$

By formulas $(h)$ and $(j)$ of the proof of Theorem 4.7 (with $\varepsilon=0$ ), rephrased as in section 5 and dualized, we see that $(m)$ and $(n)$ would be zero if $l=s+t$ were allowed in the second sums. Therefore, by an easy verification, $(m)$ and $(n)$ reduce to the following expressions, whe re $i=k+l=s+t$.

(o) $\quad\left(s-p i, \frac{r}{2}+q m-p s+p i\right) e{ }_{0} \otimes P_{*}^{\hat{i}}(x)^{p}$ with dpi $=\mathrm{ds}-\mathrm{r}$, and

(p) $\quad \alpha(q)\left(s-p i-1, \frac{r+1}{2}+q m-p s+p i\right) e_{o} \otimes P_{*}^{i} \beta(x)^{p}$ with dpi $=d s-r-p$.

Clearly $(o)$ is equal to the summand of $z^{\prime}$ involving $e_{0}$ in its first sum and $(p)$ is equal to the summand of $z^{\prime}$ involving $e_{o}$ in its second sum. Thus $y_{o}=0$ and the proof is complete.

10. The cohomology of $K(\pi, n)$ and the axiomatization of the $P^{S}$

We recall the structure of $H^{*}\left(K(\pi, n) ; z_{p}\right)=H^{* *}\left(\pi, n, z_{p}\right)$ and compute completely the mod $p$ cohomology Bockstein spectral sequence of $K(\pi, n)$ in this section. We also show (as should be well-known) that Serre's proof [23] of the axiomatization of the $\mathrm{Sq}^{\mathrm{i}}$ using $\mathrm{K}\left(\mathrm{Z}_{2}, \mathrm{n}\right)$ can be simply modified so as to apply in the case of odd primes. We shall consider only the cyclic groups $\pi=Z_{p^{t}}$, $1 \leq t \leq \infty$, where, by convention, $z_{p^{\infty}}=z$. We first fix conventions on admissible monomials relative to $t$.

Notations 10.1. (a) $p=2$. For $I=\left(s_{1}, \ldots, s_{k}\right)$, we say that $I$ is admissible if $s_{i} \geq 2 s_{i+1}$ and $s_{k} \geq 1$. The length, degree, and excess of $I$ are defined by $\ell(I)=k, d(I)=\sum s_{j}$, and, if $I=(s, J), e(I)=s-d(J)$. Define 
$P_{t}^{I}=P^{s} \ldots P^{s-1} P_{t}{ }^{s} k$, where $P_{t}^{s}=P^{s}$ if $s \geq 2, P_{t}^{1}=\beta_{t}$ if $t<\infty$, and $P_{\infty}^{1}=0$; thus, if $t=\infty$, we agree that admissibility requires $s_{k} \geq 2$. The empty sequence I is admissible, with length, degree, and excess zero, and I determines the identity operation.

(b) $p>2$. For $I=\left(\varepsilon_{1}, s_{1}, \ldots, \varepsilon_{k}, s_{k}, \varepsilon_{k+1}\right), \varepsilon_{i}=0$ or 1 , we say that $I$ is admissible if $s_{i} \geq p_{i+1}+\varepsilon_{i+1}$ and $s_{k} \geq 1$ or if $k=0$, when $I=(E)$. Define $\ell(I)=k, d(I)=\sum \varepsilon_{j}+\sum 2 s_{j}(p-1)$, and, if $I=(\varepsilon, s, J), \quad e(I)=2 s+\varepsilon-d(J)$. Define $P_{t}^{I}=\beta^{\varepsilon}{ }^{1} P^{s}{ }^{1} \ldots \beta^{\varepsilon}{ }^{\varepsilon} P^{s} k_{\beta_{t}}{ }^{\varepsilon}{ }^{k+1}$, where $\beta_{t}{ }^{0}=1$ for all $t, \beta_{t}{ }^{1}=\beta_{t}$ for $t<\infty$, and $\beta_{\infty}^{1}=0$; thus, if $t=\infty$, we agree that admissibility requires $\varepsilon_{k+1}=0$.

We now give a quick calculation of $\mathrm{H}^{*}\left(\mathrm{z}_{\mathrm{p}} \mathrm{t}, \mathrm{n}, \mathrm{z}_{\mathrm{p}}\right)$.

Lemma 10.2. $H^{*}\left(z, 1, z_{p}\right)=E\left(i_{1}\right)$ and $H^{*}\left(z, 2, z_{p}\right)=P\left(i_{2}\right)$. If $t<\infty$, then $H^{*}\left(z_{2}{ }^{t}, 1, z_{2}\right)=P\left(i_{1}\right)$, with $\beta_{t}\left(i_{1}\right)=i_{1}^{2}$, and $H^{* *}\left(z_{p}, 1, z_{p}\right)=E\left(i_{1}\right) \otimes P\left(\beta_{t}\left(i_{1}\right)\right)$ if $\mathrm{p}>2$.

Proof. $K(Z, 1)=S^{1}$ and $K(Z, 2)=C P^{\infty}$, so the first statement is obvious. For the second statement, $\mathrm{H}^{*}\left(\mathrm{z}_{\mathrm{p}} \mathrm{t}, 1, \mathrm{z}_{\mathrm{p}}\right)=\mathrm{H}^{*}\left(\mathrm{z}_{\mathrm{p}} \mathrm{t} \mathrm{z}_{\mathrm{p}}\right)$, and we can define a $\Lambda Z \mathrm{p}^{\mathrm{t}^{\text {-free }}}$ resolution of $\Lambda$, with coproduct, precisely as in Definition 1.2 (with $p$ there replaced by $p^{t}$ ) for any commutative ring $\Lambda$. The result follows by an easy computation.

Theorem 10.3. If $\mathrm{n} \geq 2$ ( $0 \mathrm{r}$ if $\mathrm{n}=1$ and either $\mathrm{p}>2$ or $\mathrm{t}<\infty$ ), then $\mathrm{H}^{*}\left(\mathrm{Z}_{\mathrm{p}} \mathrm{t}, \mathrm{n}, \mathrm{Z}_{\mathrm{p}}\right)$ is the free commutative algebra generated by the following set: $\left\{P_{t}^{I} i_{n} \mid I\right.$ is admissible and $e(I)<n$ or, if $p>2, e(I)=n$ and $\left.{ }_{1}=1\right\}$. Moreover, $H^{*}\left(z_{p t}, n, z_{p}\right)$ is a primitively generated Hopf algebra.

Proof. The lemma gives the result for $t<\infty$ and $n=1$ and for $t=\infty$ and $n=2$. Assume the result for $n-1$. Of course, the Serre spectral sequence $\left\{E_{r}\right\}$ of $K\left(Z_{p^{t}}, n-1\right) \rightarrow E \rightarrow K\left(Z_{p^{t}}, n\right), E$ acyclic, satisfies

$$
E_{2}=H^{*}\left(z_{p}, n, z_{p}\right) \otimes H^{*}\left(z_{p}, n-1, z_{p}\right) \text { and } E_{\infty}=z_{p} \text {. }
$$


First, let $p=2$; then, regarding squares as Steenrod operations, we see that we may rewrite the polynomial algebra $H^{*}\left(z_{2}, n-1, z_{2}\right)$, additively, as the exterior algebra $E(S)$, where

$$
S=\left\{P_{t} I_{n-1} \mid I \text { is admissible and } e(I)<n\right\} .
$$

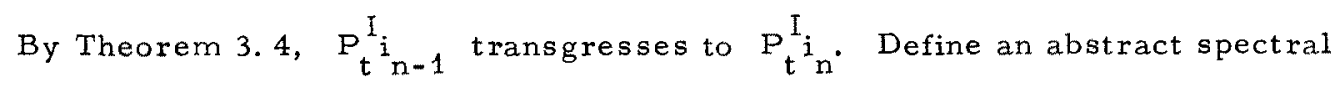
sequence of differential algebras, $\left\{E_{r}^{\prime}\right\}$, by letting $E_{2}^{\prime}=P(T S) \otimes E(S)$, where $T(S)$ is a copy of $S$ with degrees augmented by one, and by requiring $s \in S$ to transgress to $T s \in T(S)$. Clearly $E_{\infty}^{t}=Z_{2}$. Define a morphism of spectral sequences $f_{r}: E_{r}^{\prime} \rightarrow E_{r}$ by $f_{2}=g \otimes 1$, where $g \cdot P(r S) \rightarrow H^{*}\left(Z_{2} t, n, Z_{2}\right)$ is the morphism of algebras defined on generators by $g\left(\tau P_{t}{ }^{I}{ }_{n-1}\right)=P_{t} I_{n}$; clearly commutation with the differentials determines $f_{r}$ for $r>2$. Since $f_{2}{ }^{* *}$ and $f_{\infty}$ are isomorphisms, $f_{2}^{* 0}=g$ is an isomorphism by the comparison theorem $[14, p .355]$. Now let $p>2$. We may rewrite $H^{*}\left(Z_{p}, n, Z_{p}\right)$, additively, as $E(S) \otimes Q(T)$, where $Q$ denotes a truncated polynomial algebra $\left(x^{\mathrm{P}}=0\right.$ for $\left.x \in T\right)$ and where

$$
\begin{aligned}
& S=\left\{P_{t}^{I}{ }_{n-1} \mid I \text { is admissible, } e(I)<n-1, d(I)+n \text { even }\right\}, \\
& T=\left\{P_{t}^{I}{ }_{n-1} \mid I \text { is admissible, } e(I)<n, d(I)+n \text { odd }\right\} .
\end{aligned}
$$

(Note that $e(I) \equiv d(I) \bmod 2$, hence $d(I)+n$ even and $e(I)=n-1$ is impossible.) By Theorem 3.4, $P_{t}{ }^{I_{i}}{ }_{n-1}$ transgresses to $(-1)^{d(I)} P_{t} I_{i}$ and, if $\mathrm{d}(\mathrm{I})+\mathrm{n}=2 \mathrm{q}+1, \quad \mathrm{P}_{\mathrm{t}}^{\mathrm{I}_{\mathrm{n}}} \otimes\left(\mathrm{P}_{\mathrm{t}}^{\mathrm{I}_{\mathrm{i}-1}}\right)^{\mathrm{p}-1}$ transgresses to $(-1)^{\mathrm{n}} \beta \mathrm{P}^{\mathrm{q}} \mathrm{P}_{\mathrm{t}_{\mathrm{i}}}^{\mathrm{I}_{\mathrm{i}}}$. Define an abstract spectral sequence of differential algebras, $\left\{E_{r}^{\prime}\right\}$, as follows. Let

$$
E_{2}^{\prime}=[P(T S) \otimes E(\tau) \otimes P(\mu T)] \otimes[E(S) \otimes Q(T)]
$$

(the bracketed expressions are the base and fibre, respectively). Here $T S$ and $T T$ are copies of $S$ and $T$ with degrees augmented by one and $\mu T$ is a copy of $T$ with degrees multiplied by $p$ and then augmented by two. The differentials in $\left\{E_{r}^{\prime}\right\}$ are specified by requiring $s \in S$ to transgress to $T S \in T S, t \in T$ to transgress to $T t \in \tau T$, and $T t \otimes t^{p-1}$ to transgress to $\mu t \in \mu T$. An easy computation demonstrates that $E_{\infty}^{\prime}=Z_{p}$. Define a morphism of spectral sequences 
$f_{r}: E_{r}^{\prime} \longrightarrow E_{r}$ by $f_{2}=g \otimes 1$, where $g: P(\tau S) \otimes E(\tau T) \otimes P(\mu T) \rightarrow H^{*}\left(Z_{p^{t}}, n, Z_{p}\right)$ is the morphism of algebras defined on generators by $g\left(\tau P_{t}^{I_{i}}\right)=(-1)^{d(I)} P_{t} I_{i}$ and $g\left(\mu P_{t}^{I_{i}}\right)=-\beta P^{q} P_{t}{ }_{i}{ }_{n}$ if $d(I)+n=2 q+1$. As in the case $p=2$, the $f_{r}$ for $r>2$ are determined by commutation with the differentials, and $g$ is an isomorphism by the comparison theorem. The last statement follows since, by the external Cartan formula, if $\mathrm{X}$ is an H-space and $x \in H^{*}(x)$ satisfies $\psi(x)=\sum x^{\prime} \otimes x^{\prime \prime}$, then

$$
\psi P^{s}(x)=\sum_{i+j=s} \sum P^{i}\left(x^{\prime}\right) \otimes P^{j}\left(x^{\prime \prime}\right) \text { and } \psi \beta(x)=\sum\left(\beta\left(x^{\prime}\right) \otimes x^{\prime \prime}+(-1)^{\operatorname{deg} x^{\prime}} x^{\prime} \otimes \beta\left(x^{\prime \prime}\right)\right) .
$$

Thus, since $i_{n}$ and $\beta_{t}\left(i_{n}\right)$ are primitive, so are all of the $P_{t}^{I} i_{n}$.

We can now compute the mod p cohomology Bockstein spectral sequence $\left\{E_{r}\right\}$ of $K\left(Z_{p^{t}}, n\right)$. Recall that $\left\{E_{r}\right\}$ is a spectral sequence of differential algebras such that $E_{1}=H^{*}\left(p^{t}, n, z_{p}\right)$ and $E_{r+1}$ is the homology of $E_{r}$ with respect to $\beta_{r}$ for $r \geq 1$. Since $H^{*}\left(p^{t}, n, Z\right)$ is a direct sum of cyclic groups with one generator of order $\mathrm{p}^{\mathrm{r}}$ for each basis element of $\operatorname{Im}\left(\beta_{\mathrm{r}}\right) \subset E_{r}$ and one generator of infinite order for each basis element of $E_{\infty}$, the integral cohomology of $K\left(Z_{p^{t}}, n\right)$ is completely determined, additively, by $\left\{E_{r}\right\}$. If $t<\infty$ and $n=1$, Lemma 10,2 implies that $E_{1}=E_{t}$ and $E_{t+1}=E_{\infty}=Z_{p}$, hence we need only consider the case $\mathrm{n} \geq 2$.

Theorem 10.4. Let $\mathrm{n} \geq 2$. Define a subset $S$ of the set of generators for $E_{1}=H^{*}\left(z_{p}{ }^{\prime}, n, z_{p}\right)$ given in Theorem 10.3 by

(a) If $\mathrm{p}=2, \mathrm{~S}=\left\{\mathrm{P}_{\mathrm{t}} \mathrm{i}_{\mathrm{n}} \mid \mathrm{s}_{1}\right.$ and $\mathrm{d}(\mathrm{I})+\mathrm{n}$ are even and $\left.\ell(\mathrm{I})>0\right\}$.

(b) If $\mathrm{p}>2, \mathrm{~S}=\left\{\mathrm{P}_{\mathrm{t}}^{\mathrm{I}} \mathrm{i}_{\mathrm{n}} \mid \varepsilon_{1}=0, \mathrm{~d}(\mathrm{I})+\mathrm{n}\right.$ is even, and $\left.\ell(\mathrm{I})>0\right\}$.

For $y \in S$, define $z(y)=\beta(y) y+P^{2 q} \beta(y)$ if $p=2$ and degree $(y)=2 q$ and define $z(y)=\beta(y) y^{p-1}$ if $p>2$. Define an algebra $A_{r}(n, t)$ by

(c) $A_{r}(2 n, \infty)=P\left\{i_{n}\right\}$ and $A_{r}(2 n+1, \infty)=E\left\{i_{n}\right\}$.

(d) $A_{r}(2 n, t)=P\left\{i_{2 n}\right\} \otimes E\left\{\beta_{t}(2 n)\right\}$ if $r \leq t<\infty$ and 


$$
\begin{aligned}
& A_{r}(2 n, t)=P\left\{i_{2 n}^{p^{r-t}}\right\} \otimes E\left\{z\left(i_{2 n}\right) i_{2 n}^{p^{r-t}-p}\right\} \text { if } r>t \\
& \text { where } z\left(i_{2 n}\right)=\beta\left(i_{2 n}\right) i_{2 n}+P^{2 n} \beta\left(i_{2 n}\right) \text { if } p=2 \text { and } t=1 \\
& \text { and } z\left(i_{2 n}\right)=\beta_{t}\left(i_{2 n}\right) i_{2 n}^{p-1} \text { if either } p>2 \text { or } t>1
\end{aligned}
$$

(e) $\quad A_{\mathbf{r}}(2 n+1, t)=E\left\{i_{2 n+1}\right\} \otimes P\left\{\beta_{t}\left(i_{2 n+1}\right)\right\} \quad$ if $r \leq t<\infty$ and

$$
A_{r}(2 n+1, t)=z_{p} \text { if } r>t \text {. }
$$

Then, if $r \geq 1, E_{r+1}=P\left\{y^{p^{r}} \mid y \in S\right\} \otimes E\left\{z(y) y^{P^{r}-p} \mid y \in S\right\} \otimes A_{r+1}(n, t)$, $\beta_{r+1}\left(y^{p^{r}}\right)=z(y) y^{p^{r}-p}$ for $y \in S$, and $\beta_{r+t}\left(i_{2 n}^{p^{r}}\right)=z\left(i_{2 n}\right) i_{2 n}^{p^{r}}-p$

Proof. We first compute $E_{2}$ separately in the cases $p=2$ and $p>2$. Let $p=2$ and define subsets $T$ and $U$ of the set of generators of $E_{1}$ by

$$
\begin{aligned}
& \mathrm{T}=\left\{\mathrm{P}_{\mathrm{t}}^{I_{\mathrm{i}}} \mid \mathrm{s}_{\mathbf{1}} \text { is even, } \mathrm{d}(\mathrm{I})+\mathrm{n} \text { is odd, } \mathrm{e}(\mathrm{I})<\mathrm{n}-1 \text {, and } \ell(\mathrm{I})>0\right\} \\
& \mathrm{U}=\left\{\mathrm{P}_{\mathrm{t}}^{I_{\mathrm{i}}} \mid \mathrm{d}(\mathrm{I})+\mathrm{n} \text { is odd, } \mathrm{e}(\mathrm{I})=\mathrm{n}-1, \text { and } \ell(\mathrm{I})>0\right\}
\end{aligned}
$$

Recall that $\beta P^{s-1}=s P^{s}$ and observe that if $P_{t}{ }_{t}{ }_{n} \in U$, then $I=(2 q, J)$, where $\mathrm{d}(J)+\mathrm{n}=2 \mathrm{q}+1$, and $\beta \mathrm{P}_{\mathrm{t}}^{\mathrm{I}_{\mathrm{n}}}=\left(\mathrm{P}_{\mathrm{t}}^{\mathrm{J}} \mathrm{i}_{\mathrm{n}}\right)^{2}$. Let $\mathrm{C}$ be the (additive) subcomplex of $\mathrm{E}_{1}$ which is the tensor product of the following collections of subcomplexes:

(i) $P\{\beta(y)\} \otimes E\{y\}$ for $y \in T$, and

(ii) $P\left\{z^{2}\right\} \otimes E\{y\}$ for $y=P^{2 q} z \in U, \operatorname{deg}(z)=2 q+1$.

Let IC be the positive degree elements of $C$. Then $H(I C)=0$ under $\beta$, and therefore $E_{2}$ is isomorphic to $H\left(E_{1} / I C\right)$. If $y \in T U U$, then $P^{2 q} y \in U$, $\operatorname{deg} y=2 q+1$, and the refore $C$ is actually a subalgebra of $E_{1}$ and $E_{1} / I C$ is a quotient differential algebra of $E_{1}$. It is easy to see that

$$
E_{1} / I C=P\{y \mid y \in S\} \otimes E\{\beta(y) \mid y \in S\} \otimes A_{1}^{\prime}(n, t), \quad .
$$

where $A_{1}^{\prime}(n, t)$ is the quotient of the polynomial algebra generated by ${ }_{n}$ and, if $t<\infty, \beta_{t}\left(i_{n}\right)$ by the ideal generated by $i_{n}^{2}$ if $n$ is odd or by $\beta_{t}\left(i_{n}\right)^{2}$ if $n$ is even. Therefore 


$$
H\left(E_{1} / I C\right)=P\left\{y^{2} \mid y \in S\right\} \otimes E\{\beta(y) y \mid y \in S\} \otimes A_{2}^{\prime}(n, t)
$$

where $A_{2}^{\prime}(n, t)=A_{2}(n, t)$ unless $n$ is even and $t=1$, when

$A_{2}^{t}(n, t)=P\left\{i_{n}^{2}\right\} \otimes E\left\{\beta\left(i_{n}\right) i_{n}\right\}$. For $y \in S$ or $y=i_{n}$ if $n$ is even and $t=1$, $z(y)=\beta(y) y+P^{2 q} \beta(y)$, deg $y=2 q$, is a cycle in $E_{1}$ which projects to the cycle $\beta(y) y$ in $E_{1} / I C$. Since $z(y)^{2}$ bounds in $E_{1}$, it follows that $E_{2}$ has the stated form if $p=2$. Next, let $p>2$ and define a subset $T$ of the set of generators of $E_{1}$ by

$$
T=\left\{P_{t}^{I}{ }_{i n} \mid \varepsilon_{1}=0, d(I)+n \text { is odd, and } \ell(I)>0\right\} .
$$

Then, as a differential algebra, $E_{1}$ breaks up into the tensor product of the following collection of subalgebras:

(iii) $P\{y\} \otimes E\{\beta(y)\}$ for $y \in S$

(iv) $E\{y\} \otimes P\{\beta(y)\}$ for $y \in T$

(v) The free commutative algebra generated by $i_{n}$ and, if $t<\infty, \beta_{t}\left(i_{n}\right)$. The algebras in (iii) have homology $P\left\{y^{P}\right\} \otimes E\{z(y)\}$, those of (iv) are acyclic, and that of $(v)$ has homology $A_{2}(n, t)$, hence $E_{2}$ is as stated. Now assume that $E_{r+1}$ is as stated, $r \geq 1$ and any $p$. Then Proposition 6.8 computes $\beta_{r+1}$ and $E_{r+1}$ breaks up into the tensor product of $A_{r+1}(n, t)$ with subalgebras of the form $P\{x\} \otimes E\left\{\beta_{r+1}(x)\right\}$, where $x=y^{p^{r}}, y \in S$. This proves the result.

Finally, we prove the axiomatization of the $\mathrm{P}^{\mathrm{S}}$ on topological spaces. Recall first that the Cartan formula and $P^{\circ}=1$ imply that the $P^{S}$ commute with suspension $[28,30]$ and that we have shown in Proposition 8.1 that $P^{\circ}=1$ is implied by the commutation of $\mathrm{P}^{\circ}$ with $\mathrm{S}^{*}$ and the fact that $\mathrm{P}^{\circ}$ is the $\mathrm{p}$-th power on a zero dimensional class. Thus the axioms we choose (for convenience of proof) a re in fact redundant. 
Theorem 10.5. There exists a unique family $\left\{P^{s} \mid s \geq 0\right\}$ of natural homomorphisms $H^{*}\left(X ; Z_{p}\right) \rightarrow H^{*}\left(X ; Z_{p}\right)$ such that $\operatorname{deg}\left(P^{s}\right)=s$ if $p=2$, $\operatorname{deg}\left(P^{s}\right)=2 s(p-1)$ if $p>2$, and

(i) $\mathrm{P}^{\circ}$ is the identity homomorphism

(ii) $P^{s}(x)=x^{p}$ if $p=2$ and $s=\operatorname{deg} x$ or $p>2$ and $2 s=\operatorname{deg} x$

(iii) $P^{s}(x)=0$ if $p=2$ and $s>\operatorname{deg} x$ or $p>2$ and $2 s>\operatorname{deg} x$

(iv) $\quad P^{s}(x \otimes y)=\sum_{i+j=s} P^{i}(x) \otimes P^{j}(y)$ for $x \otimes y \in H^{*}(X \times Y)$

(v) $\sigma^{*} P^{s}=P^{s} \sigma^{*}$, where $\sigma^{*}$ is the suspension of a fibration.

Proof. Suppose given $\left\{R^{s} \mid s \geq 0\right\}$ which also satisfy the axioms. If $x \in H^{n}\left(x, z_{p}\right)$, then $x=f^{*}\left(i_{n}\right)$ for some $f: X \rightarrow K\left(z_{p}, n\right)$, hence it suffices to prove that $P^{s}\left(i_{n}\right)=R^{s}\left(i_{n}\right)$. The result is obvious from (i), (ii), and (iii) if $n=1$ or if $p>2$ and $n=2$. Assume that $P^{s}\left(i_{n-1}\right)=R^{s}\left(i_{n-1}\right)$ for all $s$ and consider $y=P^{s}\left(i_{n}\right)-R^{s}\left(i_{n}\right), 0<s<n$ if $p=2$ and $0<2 s<n$ if $p>2$. By $(v), \sigma^{*}(y)=0$, where

$$
\sigma^{*}: H^{* *}\left(Z_{p}, n, Z_{p}\right) \rightarrow H^{*}\left(Z_{p}, n-1, Z_{p}\right)
$$

If $\mathrm{p}=2, \sigma^{*}$ is an isomorphism in degrees less than $2 \mathrm{n}$ and therefore $\mathrm{y}=0$. Let $p>2$. As shown in the proof of Theorem 10.3, (i) and (iv) imply that both $P^{S}\left(i_{n}\right)$ and $R^{S}\left(i_{n}\right)$ are primitive. By Theorem 10.3, we see that

$$
\left\{\mathrm{P}_{i_{n}} \mid \mathrm{I} \text { admissible, } e(I) \leq n\right\}
$$

is a basis for the primitive elements of $\mathrm{H}^{*}\left(\mathrm{Z}_{\mathrm{p}}, \mathrm{n}, \mathrm{Z}_{\mathrm{p}}\right)$. The only elements of this set which are in Ker $\sigma^{*}$ are $p$-th powers and elements of the form $\beta P^{q}(y)$, deg $y=2 q+1$, which have degree $2 p q+2$. If $n$ is odd, then $y$ has odd degree and is thus zero. If $\mathrm{n}$ is even, then all primitive elements in Ker $\sigma^{*}$ have degree at least pn, which is greater than the degree of $y$, and again $y=0$. 


\section{Cocommutative Hopf algebras}

In this section, we consider the following category $\zeta$. The objects of $\zeta$ are triples $C=(E, A, F)$ where $A$ is a ( $Z$-graded) cocommutative Hopf algebra over a commutative ring $\Lambda, E$ is a right and $F$ is a left (Z-graded) cocommutative A-coalgebra. Thus $E$ and $F$ are $A$-modules and cocommutative coalgebras (not necessarily unital or augmented), and their coproducts $\psi$ are morphisms of A-modules. We say that $C$ is unital if $E$ and $F$ are unital and augmented and their units and augmentations are morphisms of A-modules. A morphism $\gamma: C \rightarrow C^{\prime}$ in $\zeta^{\circ}$ is a triple $\gamma=(\alpha, \lambda, \beta)$, where $\lambda: A \rightarrow A^{\prime}$ is a morphism of Hopf algebras and $\alpha: E \rightarrow E^{\prime}$ and $\beta: F \rightarrow F^{\prime}$ are $\lambda$-equivariant morphisms of coalgebras; thus $\alpha(e a)=\alpha(e) \lambda(a)$ and $\beta(a f)=\lambda(a) \beta(f)$ for $e \in E$, a $\in A$, and $f \in F$. We say that $\gamma$ is unital if $\alpha$ and $\beta$ are morphisms of unital augmented coalgebras. For $C$ and $C^{\prime}$ in $\zeta$, define $C \otimes C^{\prime}=\left(E \otimes E^{\prime}, A \otimes A^{\prime}\right.$, $\left.F \otimes F^{\prime}\right) \epsilon \zeta$ and observe that

$$
\psi=(\psi, \psi, \psi): C=(E, A, F) \longrightarrow(E \otimes E, A \otimes A, F \otimes F)=C \otimes C
$$

is a morphism in $\zeta$; clearly $\psi$ is unital if $C$ is unital. Define homology and cohomology functors on the category $\zeta$ by

$$
\mathrm{H}_{\mathrm{st}}(\mathrm{C})=\operatorname{Tor}_{\text {st }}^{(\mathrm{A}, \Lambda)}(\mathrm{E}, \mathrm{F}) \text { and } \mathrm{H}^{\mathrm{st}}(\mathrm{C})=\mathrm{Ext}_{(\mathrm{A}, \Lambda)}^{\text {st }}\left(\mathrm{E}, \mathrm{F}^{*}\right) \text {. }
$$

We shall define and study Steenrod operations on $H^{*}(C)$ when $\Lambda=z_{p}$. The results here generalize work of Liulevicius [13].

In the following definitions, we recall the description of $H^{*}(C)$, with its product, in terms of the bar construction.

Definitions 11.1. For $C=(E, A, F) \in \zeta$, let $\bar{C}=(A, A, F) \in \zeta$.

Let $J A$ be the cokernel of the unit $\Lambda \rightarrow A$. Define the bax construction $B(C)$ as follows. $B(C)=E \otimes T(J A) \otimes F$ as a $\Lambda$-module, where $T(J A)$ is the tensor algebra on JA. Write elements of $B(C)$ in the form $e\left[a_{1}|\ldots| a_{s}\right] f ;$ such an element has homological degree $s$, internal degree $t=\operatorname{deg} e+\sum \operatorname{deg} a_{i}+\operatorname{deg} f$, 
and total degree stt. Define $\varepsilon: B(C) \rightarrow E \otimes_{A} F$ and $d: B_{s, *}(C) \rightarrow B_{s-1, *}(C)$ by

$$
\begin{aligned}
\varepsilon(e[] f)=e \otimes f, & \varepsilon\left(e\left[a_{1}|\ldots| a_{s}\right] f\right)=0 \text {, and } \\
d\left(e\left[a_{1}|\ldots| a_{s}\right] f\right)= & -\bar{e}_{1}\left[a_{2}|\ldots| a_{s}\right] f \\
& -\sum_{i=1}^{s-1} \bar{e}\left[\bar{a}_{1}|\ldots| \bar{a}_{i-1}\left|\bar{a}_{i} a_{i+1}\right| a_{i+2}|\ldots| a_{s}\right] f \\
& -\bar{e}\left[\bar{a}_{1}|\ldots| \bar{a}_{s-1}\right] a_{s} f, \text { where } \bar{x}=(-1)^{1+\operatorname{deg} x} x .
\end{aligned}
$$

If $E=A$, then $d$ is a morphism of left $A$-modules and $d S+S d=1-\sigma \varepsilon$, where $\sigma: F \rightarrow B(\bar{C})$ and $S: B_{S, *}(\bar{C}) \rightarrow B_{S+1, *}(\bar{C})$ are defined by the formulas

$$
\sigma(f)=[] f \text { and } S\left(a\left[a_{1}|\ldots| a_{s}\right] f\right)=\left[a\left|a_{1}\right| \ldots \mid a_{s}\right] f
$$

Clearly $d=1 \otimes_{A} d$ on $B(C)=E \otimes_{A} B(\bar{C})$. By adjoint associativity,

$$
\operatorname{Hom}_{A}\left(B(\bar{C}), E^{*}\right) \cong B^{*}(C) \cong \operatorname{Hom}_{A}\left(B(E, A, A), F^{*}\right)
$$

Therefore (1) admits the equivalent reformulation

$$
H_{*}(C)=H(B(C)) \text { and } H^{*}(C)=H\left(B^{*}(C)\right)=\operatorname{Ext}(A, \Lambda)\left(F, E^{*}\right)
$$

Definitions 11.2, Let $C$ and $C^{\prime}$ be objects of $\zeta$. Define the AlexanderWhitney map $\xi: B\left(C \otimes C^{\prime}\right) \rightarrow B(C) \otimes B\left(C^{\prime}\right)$ and the shuffle map $\eta: B(C) \otimes B\left(C^{\prime}\right) \rightarrow B\left(C \otimes C^{\prime}\right)$ by the formulas

$$
\begin{aligned}
& \xi\left(e \otimes e^{\prime}\left[a_{1} \otimes a_{1}^{\prime}|\ldots| a_{s} \otimes a_{s}^{\prime}\right] f \otimes f^{\prime}\right) \\
& =\sum_{k=0}^{s}(-1)^{\mu(k)} e\left[a_{1}|\ldots| a_{k}\right] a_{k+1} \ldots a_{s} f \otimes e^{\prime} a_{1}^{\prime} \ldots a_{k}^{\prime}\left[a_{k+1}^{\prime}|\ldots| a_{s}^{\prime}\right] f^{\prime}, \\
& \text { where } \mu(k)=\operatorname{deg} e^{\prime}\left(k+\operatorname{deg} a_{1} \ldots a_{s} f\right)+\sum_{i=1}^{k} \operatorname{deg} a_{j}^{\prime}\left(k-i+\operatorname{deg} a_{i+1} \ldots a_{s} f\right) \\
& \quad+\sum_{j=k+1}^{s}\left(1+\operatorname{deg} a_{j}^{\prime}\right) \operatorname{deg} a_{j+1} \ldots a_{s} f, \text { and }
\end{aligned}
$$$$
\text { (7) } \quad \eta\left(e\left[a_{1}|\ldots| a_{s}\right] f \otimes e^{\prime}\left[a_{s+1}|\ldots| a_{s+t}\right] f^{\prime}\right)
$$$$
=\sum_{\pi}(-1)^{\nu(\pi)} e \otimes e^{\prime}\left[a_{\pi(1)}|\ldots| a_{\pi(s+t)}\right] f \otimes f^{\prime} \text {, }
$$

where $a_{i} \in A$ if $i \leq s, a_{i} \in A^{\prime}$ if $i>s$, the sum is taken over all $(s, t)$-shuffles $\pi(\operatorname{see}[15, p, 17])$, and 


$$
v(\pi)=\sum_{\pi(i)>\pi(s+j)}\left(1+\operatorname{deg} a_{i}\right)\left(1+\operatorname{deg} a_{s+j}\right) .
$$

The unnormalized bar construction $E \otimes T(A) \otimes F$ admits a structure of simplicial graded $\Lambda$-module under which $\xi$ and $\eta$ are in fact the classical normalized Alexander-Whitney and shuffle maps. Define $D=\xi B(\psi): B(C) \rightarrow B(C) \otimes B(C)$. Then $D$ gives $B(C)$ a structure of coassociative coalgebra; if $C$ is unital, then $B(C)$ is unital and augmented. If $E=A$, then $D$ coincides with the morphism of left A-modules defined inductively by

$$
\begin{aligned}
& D([] f)=\sum[] f^{\prime} \otimes[] f^{\prime \prime} \text { if } \psi(f)=\sum f^{\prime} \otimes f^{\prime \prime}, \text { and } \\
& D S=S D, \text { where } S=S \otimes 1+\sigma \epsilon \otimes S \text { on } B(\bar{C}) \otimes B(\bar{C}) .
\end{aligned}
$$

Clearly $D$ on $B(C)=E \otimes_{A} B(\bar{C})$ is the composite

$$
E \otimes_{A} B(\bar{C}) \stackrel{\psi \otimes D}{\longrightarrow} E \otimes E \otimes_{A} B(\bar{C}) \otimes B(\bar{C}) \stackrel{1 \otimes T \otimes 1}{\longrightarrow} E \otimes_{A} B(\bar{C}) \otimes E \otimes_{A} B(\bar{C}) .
$$

We define the cup product on $B^{*}(C)$ to be the composite

$$
u: \mathrm{B}^{*}(\mathrm{C}) \otimes \mathrm{B}^{*}(\mathrm{C}) \stackrel{\alpha}{\longrightarrow}[\mathrm{B}(\mathrm{C}) \otimes \mathrm{B}(\mathrm{C})]^{*} \stackrel{\mathrm{D}^{*}}{\longrightarrow} \mathrm{B}^{*}(\mathrm{C})
$$

We have the following analog of Lemma 7.1; a more precise analog (in terms of $\xi$ ) could also be proven, and an alternative proof by semi-simplicial rather than homological techniques is available.

Lemma 11.3. Let $\pi$ be a subgroup of $\Sigma_{r}$ and let $W$ be a $\Lambda \pi$-free resolution of $\Lambda$ such that $w_{0}=\Lambda \pi$ with $\Lambda \pi$ generator $e_{0}$. Let $C \in \zeta$. Bigrade $W \otimes B(C)$ by $[W \otimes B(C)]_{s t}=\sum_{i+j=s} W_{i} \otimes B_{j t}(C)$. Then there exists a morphism of bigraded $\Lambda \pi$-complexes $\Delta: \mathrm{W} \otimes \mathrm{B}(\mathrm{C}) \rightarrow \mathrm{B}(\mathrm{C})^{\mathbf{r}}$ which is natural in $\mathrm{C}$ and satisfies the following properties:

(i) $\Delta(w \otimes b)=0$ if $b \in B_{0, *}(C)$ and $w \in W_{i}$ for $i>0$

(ii) $\Delta\left(\mathrm{e}_{\mathrm{o}} \otimes \mathrm{b}\right)=\mathrm{D}(\mathrm{b})$ if $\mathrm{b} \in \mathrm{B}(\mathrm{C})$, where $\mathrm{D}$ is the iterated coproduct

(iii) If $E=A$, then $\Delta$ is a morphism of left A-modules, where $A$ operates 
on $W \otimes B(C)$ by $a(w \otimes b)=(-1)^{\operatorname{deg} w \operatorname{deg} a} w \otimes a b$.

(iv)

$$
\Delta\left(\mathrm{W}_{\mathrm{i}} \otimes \mathrm{B}_{\mathrm{st}}(\mathrm{C})\right)=0 \text { if } \mathrm{i}>(\mathrm{r}-1) \mathrm{s} .
$$

Moreover, any two such $\Delta$ are naturally $\Lambda \pi$-homotopic.

Proof. Observe first that the cocommutativity of A ensures that (iii) is compatible with the $\pi$-equivariance of $\Delta$. Observe next that it suffices to prove the result when $E=A$, since we can then define $\Delta$ on $W \otimes B(C)=E \otimes A \otimes B(\bar{C})$ to be the composite

$$
E \otimes_{A} W \otimes B(\bar{C}) \stackrel{\psi \otimes \Delta}{\longrightarrow} E^{r} \otimes_{A} B(\bar{C})^{r} \stackrel{U}{\longrightarrow}\left[E \otimes_{A} B(\bar{C})\right]^{r}
$$

where $U$ is the evident shuffle. We define $\Delta$ on $W_{i} \otimes B_{s t}(\bar{C})$ by induction on $i$ and for fixed $i$ by induction on $s$. Formula (i) defines $\Delta$ for $s=0$ and all $i>0$ and formula (ii) and $\pi$-equivariance defines $\Delta$ for $i=0$ and all $s$. Let $i \geq 1$ and $s \geq 1$ and assume that $\Delta$ has been defined for $i^{\prime}<i$ and for our given $i$ and $s^{\prime}<s$. Let $\left\{w_{k}\right\}$ be a $\Lambda \pi$-basis for $w_{i}$. By (iii) and $\pi$-equivariance, it suffices to define $\Delta(\mathrm{w} \otimes \mathrm{S}(\mathrm{y}))$ for $\mathrm{w} \in\left\{\mathrm{w}_{\mathrm{k}}\right\}$ and $\mathrm{y} \in \mathrm{B}_{\mathrm{s}-1, \mathrm{w}_{\mathrm{F}}}(\overline{\mathrm{C}})$. Let $S=\sum_{i=1}^{r}(\sigma \varepsilon)^{i} \otimes S \otimes 1^{r-i-1}$ on $B(\bar{C})^{r}$. Then $d S+S d=1-(\sigma \varepsilon)^{r}$. We define (v) $\quad \Delta(\mathrm{w} \otimes \mathrm{S}(\mathrm{y}))=(-1)^{\mathrm{deg} w} \mathrm{~S} \Delta(\mathrm{w} \otimes \mathrm{y})+\mathrm{S} \Delta(\mathrm{d}(\mathrm{w}) \otimes \mathrm{S}(\mathrm{y}))$.

Observe that $(v)$ is equivalent to (ii) on $w=e_{0}$ and that $(v)$ is well-defined by the induction hypothesis, To verify that $d \Delta=\Delta d$, write $(v)$ in the form $\Delta(1 \otimes S)=S \Delta(1 \otimes 1+\mathrm{d} \otimes \mathrm{S}) . \quad T$ hen:

$$
\begin{aligned}
\mathrm{d} \Delta(1 \otimes \mathrm{S}) & =\mathrm{dS} \Delta(1 \otimes 1+\mathrm{d} \otimes \mathrm{S})=(1-\mathrm{Sd}) \Delta(1 \otimes 1+\mathrm{d} \otimes \mathrm{S}) \\
& =[\Delta-\mathrm{S} \Delta(\mathrm{d} \otimes 1+1 \otimes \mathrm{d})](1 \otimes 1+\mathrm{d} \otimes \mathrm{S}) \\
& =\Delta+\Delta(\mathrm{d} \otimes \mathrm{S})-\mathrm{S} \Delta(\mathrm{d} \otimes 1)-\mathrm{S} \Delta(1 \otimes \mathrm{d})+\mathrm{S} \Delta(\mathrm{d} \otimes \mathrm{dS}) \\
& =\Delta+\Delta(\mathrm{d} \otimes \mathrm{S})-\mathrm{S} \Delta(\mathrm{d} \otimes 1)-\mathrm{S} \Delta(1 \otimes \mathrm{d})+\mathrm{S} \Delta(\mathrm{d} \otimes 1)-\mathrm{S} \Delta(\mathrm{d} \otimes \mathrm{Sd}) \\
& =\Delta+\Delta(\mathrm{d} \otimes \mathrm{S})-\Delta(1 \otimes \mathrm{dS})=\Delta+\Delta(\mathrm{d} \otimes \mathrm{S})-\Delta+\Delta(1 \otimes \mathrm{dS}) \\
& =\Delta(\mathrm{d} \otimes 1+1 \otimes \mathrm{d})(1 \otimes \mathrm{S})
\end{aligned}
$$

(where no terms involving $\sigma \varepsilon$ are relevant by (i) and an easy verification). Thus 
(i), (ii), (iii), and (v), together with $\pi$-equivariance, provide an explicit construction of a natural morphism $\Delta$ of $\Lambda \pi$-complexes, To see that (iv) holds, observe that if $w \in\left\{w_{k}\right\} \subset w_{i}$ and $y=a_{1}\left[a_{2}|\ldots| a_{s}\right] f$, then $\Delta(w \otimes S(y))$ is a linear combination in $B(\bar{C})^{r}$ of terms involving precisely the factors $a_{i}(j)$ and $f^{(j)}$ in the $B(\bar{C})$, where $\psi\left(a_{i}\right)=\sum a_{i}^{(1)} \otimes \ldots \otimes a_{i}^{(r)}$ and $\psi(f)=\sum f^{(1)} \otimes \ldots \otimes f^{(r)}$ give the iterated coproducts. Thus no summand of $\Delta(\mathrm{w} \otimes \mathrm{S}(\mathrm{y}))$ can have homological degree greater than $r s$. Since $\Delta(w \otimes S(y))$ has homological degree $i t s>r s$ if $i>(r-1) s$, this proves (iv). The uniqueness of $\Delta$ up to $\Lambda \pi$-homotopy follows easily by use of the contracting homotopy $S$ on $B(\bar{C})^{r}$.

We now pass to the category $P(\pi, \infty, \Lambda)$ of Defitions 2.1 .

Definition 11.4. Let $\mathrm{C} \in \zeta$. Let $\alpha: \mathrm{B}^{* *}(\mathrm{C})^{\mathrm{r}} \rightarrow\left[\mathrm{B}(\mathrm{C})^{\mathrm{r}}\right]^{*}$ be the natural map and define a $\Lambda \pi$-morphism $\theta: W \otimes B^{*}(C) \rightarrow B^{*}(C)^{r}$ by the formula

$$
\theta(w \otimes x)(k)=(-1)^{\operatorname{deg} w \operatorname{deg} x}{ }_{\alpha(x) \Delta(w \otimes k),} w \in W, \quad x \in B^{*}(C)^{r}, k \in B(C) .
$$

Since $\theta$ may be defined for $\pi=\Sigma_{r}$ and then factored through $j: W \rightarrow V$ as in Definition 2.1, and the resulting composite is naturally $\Lambda \pi$-homotopic to the original $\theta$ defined in terms of $W, \theta$ satisfies condition (ii) of Definition 2.1. By the lemma, formula (11) specializes to give

$$
\begin{aligned}
& \theta\left(e_{0} \otimes x\right)=D^{*} \alpha(x) \text { for any } x \in B^{*}(C)^{r} \text { and } \\
& \theta(w \otimes x)=\varepsilon(w) D^{*} \alpha(x) \text { for any } x \in B^{\circ}, *(C)^{r} \text { and } w \in W .
\end{aligned}
$$

By (10) and (12), $\theta$ satisfies condition (i) of Definition 2.1. Since $\theta$ is natural on morphisms in $\zeta$, we thus obtain a functor $\Gamma: \zeta \rightarrow \gamma(\pi, \infty, \Lambda)$ by setting $\Gamma(C)=\left(B^{*}(C), \theta\right)$ on objects and $\Gamma(\gamma)=B^{*}(\gamma)$ on morphisms. By (13), if $C$ is unital in $\zeta^{\circ}$, then $\Gamma(C)$ is unital in $\gamma(\pi, \infty, \Lambda)$. If $\Lambda=z_{p}$, $\pi$ is cyclic of order $p$, and $C=\tilde{C} \otimes Z_{p}$ where $\widetilde{C}$ is $Z$-free (that is, $\tilde{E}, \tilde{A}$, and $\tilde{F}$ are $Z$-free), then we agree to choose $\theta$ for $C$ to be the mod $p$ reduction of $\theta$ for $C ; \Gamma(C)$ will thus be reduced $\bmod p$. Note that if $x \in B^{*}(C)$ has bidegree $(s, t)$, then $\theta(w \otimes x)$ has bidegree $(s-\operatorname{deg} w, t) . W \otimes B^{*}(C)^{r}$ and $B^{*}(C)$ should be thought of 
as regarded by total degree in defining the functor $\Gamma$.

Observe that, by Definition 6.1, we now have $u_{i}$-products in $B^{* / *}(C)$ for any $\mathrm{C} \in \zeta$. When $\Lambda=\mathrm{z}_{\mathrm{p}}$, the results of Proposition 2.3 will clearly apply to the Steenrod operations in $H^{*}(C)$, and the following lemmas will imply the applicability of the external Cartan formula and the Adem relations.

Lemma 11.5. For any objects $C$ and $C^{\prime}$ in $\zeta$, the following diagram is $\Lambda \pi$-homotopy commutative.

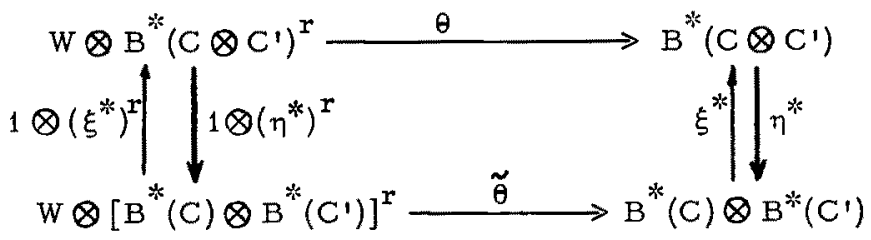

Proof. It suffices to prove the $\Lambda \pi$-homotopy commutativity of the diagram

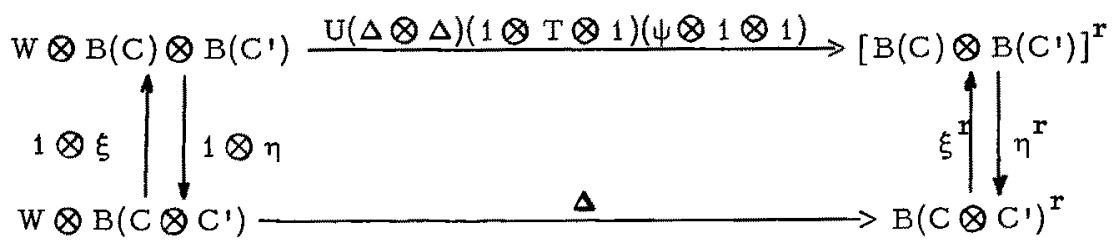

and this diagram need only be studied with $C$ and $C^{\prime}$ replaced by $\bar{C}$ and $\bar{C}^{\prime}$. Since $B\left(\bar{C} \otimes \bar{C}^{\prime}\right)^{r}$ and $\left[B(\bar{C}) \otimes B\left(\bar{C}^{\prime}\right)\right]^{r}$ have obvious contracting homotopies, the result follows by an easy double induction like that in the proof of Lemma 11.3.

Corollary 11.6. If $C \in \zeta$, then $\Gamma(C)$ is a Cartan object of $\zeta(\pi, \infty, \Lambda)$ Lemma 11.7. If $C \in \zeta, \Lambda=z_{p}$, then $\Gamma(C)$ is an Adem object of $\zeta(p, \infty)$. Proof. Precisely as in the proof of Lemma 7.8, it suffices to prove the $\tau$-homotopy commutativity of the following diagram: 


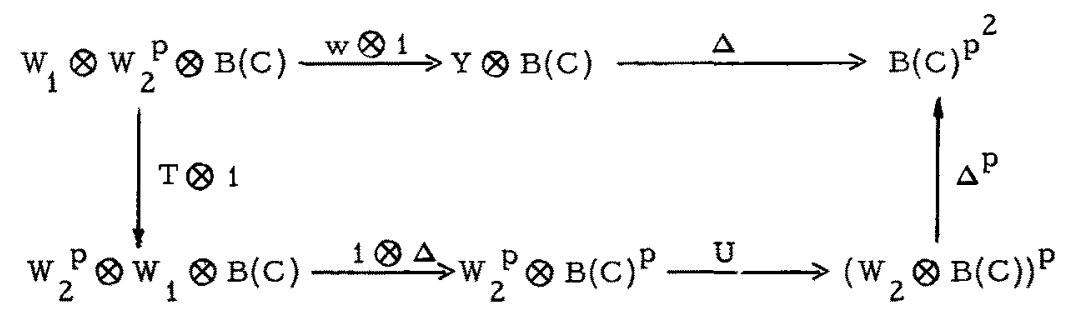

We need only consider this diagram with $C$ replaced by $\bar{C}$, and, since $B(\bar{C})^{p^{2}}$ has a contracting homotopy, the result then holds by another easy double induction.

The following theorem summarizes the properties of the $P^{s}$ and $\beta P^{s}$ on $\mathrm{H}^{*}(\mathrm{C})$ for $\mathrm{C} \in \zeta, \Lambda=\mathrm{Z}_{\mathrm{p}^{*}}$. We shall be very precise as to grading since there is considerable confusion on this point in the literature. We are thinking of $\mathrm{H}^{*}(\mathrm{C})$ as regraded by total degree in applying our general theory. An alternative formulation that is sometimes convenient will be given after the theorem.

Theorem 11.8. Let $C \in \mathcal{C}, \Lambda=z_{\mathrm{p}}$. Then there exist natural homomorphisms $\mathrm{P}^{\mathrm{i}}$ and, if $\mathrm{p}>2, \beta \mathrm{P}^{\mathrm{i}}$ defined on $\mathrm{H}^{*}(\mathrm{C})$, with

(a) $\mathrm{P}^{\mathrm{i}}: \mathrm{H}^{s, t}(\mathrm{C}) \longrightarrow \mathrm{H}^{\mathrm{s}+\mathrm{i}-\mathrm{t}, 2 \mathrm{t}}(\mathrm{C})$ if $\mathrm{p}=2$;

(b) $\quad \mathrm{P}^{\mathrm{i}}: \mathrm{H}^{\mathrm{st}}(\mathrm{C}) \longrightarrow \mathrm{H}^{\mathrm{s}}+(2 \mathrm{i}-\mathrm{t})(\mathrm{p}-1), \mathrm{pt}(\mathrm{C})$ and $\beta P^{i}: H^{s t}(C) \longrightarrow H^{s+1+(2 i-t)(p-1), p t}(C)$ if $p>2$.

These operations satisfy the following properties:

$$
\begin{aligned}
& \mathrm{p}^{\mathrm{i}}=0 \text { if } \mathrm{p}=2 \text { and either } \mathrm{i}<\mathrm{t} \text { or } \mathrm{i}>\mathrm{s}+\mathrm{t} \\
& \mathrm{P}^{\mathrm{i}}=0 \text { if } \mathrm{p}>2 \text { and either } 2 \mathrm{i}<\mathrm{t} \text { or } 2 \mathrm{i}>\mathrm{s}+\mathrm{t} \\
& \beta \mathrm{P}^{\mathrm{i}}=0 \text { if } \mathrm{p}>2 \text { and either } 2 \mathrm{i}<\mathrm{t} \text { or } 2 \mathrm{i} \geq \mathrm{s}+\mathrm{t}
\end{aligned}
$$

(ii) $P^{i}(x)=x^{p}$ if $p=2$ and $i=s+t$ or if $p>2$ and $2 i=s+t$

(iii) If $\mathrm{C}=\ddot{C} \otimes Z_{p}$, where $\tilde{C}$ is $Z$-free, then $\beta P^{i-1}=i P^{i}$ if $p=2$ and $\beta P^{i}$ is the composition of $\beta$ and $P^{i}$ if $p>2$.

(iv) $\quad P^{j}=\sum P^{i} \otimes P^{j-i}$ and $\beta P^{j}=\sum\left(\beta P^{i} \otimes P^{j-i}+P^{i} \otimes \beta P^{j-i}\right)$ or $H^{*}\left(C \otimes C^{\prime}\right)$; the internal Cartan formula is satisfied in $\mathrm{H}^{*}(\mathrm{C})$. 
(v) If $\gamma: \mathrm{C}^{\prime} \rightarrow \mathrm{C}$ and $\phi: \mathrm{C} \longrightarrow \mathrm{C}^{\prime \prime}$ are unital morphisms in $\zeta$ such that $\phi_{Y}=0$ on the cokernels of the units, then $\sigma P^{i}=P^{i}$ and $\sigma \beta P^{i}=-\beta P^{i}$, where $\sigma: \mathrm{H}^{\mathbf{s t}}\left(\mathrm{C}^{\prime \prime}\right) \longrightarrow \mathrm{H}^{\mathrm{s}-1, \mathrm{t}}\left(\mathrm{C}^{\prime}\right)$ is the suspension.

(vi) The $\mathrm{P}^{\mathrm{i}}$ and $\beta \mathrm{P}^{\mathrm{i}}$ satisfy the Adem relations as stated in Corollary 5.1 . Proof. If $x \in H^{s t}(C)$, then $D_{i}(x)=\theta_{x_{k}}\left(e_{i} \otimes x^{p}\right) \in H^{p s-i, p t}(C)$. The $P^{i}$ and $\beta P^{i}$ are defined by formulas (5.1) and (5.2), with $x$ having its total degree $q=s+t$; thus $(a)$ and $(b)$ a re valid. The vanishing of $P^{i}(x)$ for $i<t$ if $p=2$ and of $\beta^{\varepsilon} P^{i}(x)$ for $2 i<t$ if $p>2$ follows from part (iv) of Lemma 11.3. The remainder of the theorem follows immediately from our general theory and the previous lemmas. For (v), note that the composite $\mathrm{B}^{*}\left(\mathrm{C}^{\prime \prime}\right) \stackrel{\mathrm{B}^{*}(\emptyset)}{\longrightarrow} \mathrm{B}^{*}(\mathrm{C}) \stackrel{\mathrm{B}^{*}(\gamma)}{\longrightarrow} \mathrm{B}^{*}\left(\mathrm{C}^{\prime}\right)$ is zero on the kernel of the augmentation $B^{*}\left(C^{\prime \prime}\right) \rightarrow Z_{p^{*}}$ An alternative formulation of $(v)$ in the non-unital case can easily be obtained.

In addition to (v), the Kudo transgression theorem, Theorem 3.4, applies to appropriate spectral sequences involving objects of $\zeta$. The hypothesis of (iii) is seldom satisfied in practice, and $\beta P^{i}$ is generally an independent operation having nothing to do with any Bockstein. There is an alternative definition of the operations, which amounts to the following regrading of our operations. Define (c) $\tilde{\mathrm{P}}^{\mathrm{i}}=\mathrm{Sq}^{\mathrm{i}}=\mathrm{P}^{\mathrm{i}+\mathrm{t}}: \mathrm{H}^{\mathrm{st}}(\mathrm{C}) \longrightarrow \mathrm{H}^{\mathrm{s}+\mathrm{i}, 2 \mathrm{t}}(\mathrm{C})$ if $\mathrm{p}=2$; (d) $\quad \tilde{P}^{i}=P^{i+t}: H^{s, 2 t}(C) \longrightarrow H^{s+2 i(p-1), 2 p t}(C)$ and $\beta \tilde{P}^{i}=\beta P^{i+t}: H^{s, 2 t}(C) \longrightarrow H^{8+2 i(p-1)+1,2 p t}(C)$ if $p>2$.

This regrading is reasonable if $p=2$, but has the effect of eliminating all operations on $\mathrm{H}^{\mathrm{st}}(\mathrm{C})$ for $\mathrm{t}$ odd if $\mathrm{p}>2$; of course, these operations are non-trivial since, if $s$ and $t$ are odd, the $p$-th power operation on $H^{s t}(C)$ is non-trivial in general. The results of the theorem can easily be transcribed for the $\check{P}^{i}$ and $\beta \tilde{P}^{i}$; for example, the Adem relations are still correct precisely as stated but with all $P^{i}$ and $\beta P^{i}$ replaced by $\tilde{P}^{i}$ and $\beta \tilde{P}^{i}$. The motivation for the reindexing is just the desire to make $\tilde{P}^{\circ}$ the first non-trivial operation. This operation is of 
particular importance in the applications, and we now evaluate it.

Definition 11.9. Let $C=(E, A, F) \in \zeta$, where $E, A$, and $F$ are positively graded and of finite type. Then $B^{*}(C)$ may be identified with $E^{*} \otimes T\left(I A^{*}\right) \otimes F^{*}$. Define $\lambda: B^{s t}(C) \longrightarrow B^{s, p t}(C)$ by

$$
\lambda\left(\varepsilon\left[\alpha_{1}|\ldots| \alpha_{s}\right] \phi\right)=\varepsilon^{\mathrm{P}}\left[\alpha_{1}^{\mathrm{p}}|\ldots| \alpha_{\mathrm{s}}^{\mathrm{p}}\right] \phi^{\mathrm{p}} .
$$

Then $\lambda$ commutes with the differential and induces $\lambda_{*}: \mathrm{H}^{\mathrm{st}}(\mathrm{C}) \rightarrow \mathrm{H}^{\mathrm{s}, \mathrm{pt}}(\mathrm{C})$. Of course, if $p>2$, then $\lambda\left(\varepsilon\left[\alpha_{1}|\ldots| \alpha_{s}\right] \emptyset\right)=0$ if $\varepsilon, \alpha_{i}$, or $\varnothing$ has odd degree and thus $\lambda_{*}=0$ if $t$ is odd.

Proposition 11.10. Let $\mathrm{C}=(E, \mathrm{~A}, \mathrm{~F}) \in \zeta$, where $E, A$, and $F$ are positively graded and of finite type. Let $x \in H^{s, t}(C)$ where $t$ is even if $p>2$. Then $\tilde{\mathrm{P}}^{\circ}(\mathbf{x})=\lambda_{*}(\mathbf{x})$.

Proof. Let $y=e\left[a_{1}|\ldots| a_{s}\right] f \in B_{s, p t}(C)$. A straightforward, but tedious, calculation demonstrates that

$$
\Delta\left(e_{s(p-1)} \otimes y\right)=\sum(-1)^{m s} v(-s)^{-1}\left(e^{\prime}\left[a_{1}^{\prime}|\ldots| a_{s}^{\prime}\right] f^{\prime}\right)+N z
$$

where the sum is taken over the symmetric summands $e^{\prime} \otimes \ldots \otimes e^{\prime}, a_{i}^{\prime} \otimes \ldots \otimes a_{i}^{\prime}$, and $f^{\prime} \otimes \ldots \otimes f^{\prime}$ of the iterated coproducts. (A moment's reflection on the case $C=\left(z_{p}, Z_{p} G, z_{p}\right)$, where $G$ is a group, and a glance at Lemma 8.2 should convince the reader of the plausibility of this statement.) The result now follows easily from the definitions.

Remarks 11.11. If $p>2$, then it $c$ an be shown by a tedious calculation that $-\beta \tilde{P}^{o}(x)=\langle x\rangle^{p}$ for $x \in H^{1,2 t}(C)$, where $C=\left(z_{p}, A, z_{p}\right) \in \zeta$ and $\langle x\rangle^{p}$ is as defined in Remarks 6.9. It is possible that $-\beta \tilde{P} S(x)=\langle x\rangle^{p}$ for $x \in H^{2 s+1,2 t}(C)$ and any $\mathrm{C} \in \zeta$, but this appears to be difficult to prove. 


\section{Bibliography}

1. J.F. Adams, On the structure and applications of the Steenrod algebra. Comment. Math. Helv., 1958.

2. J. Adem, The relations on Steenrod powers of cohomology classes. Algebraic geometry and topology. A symposium in honor of S. Lefschetz, 1957.

3. S. Araki and T. Kudo, Topology of $\mathrm{H}_{\mathrm{n}}$-spaces and $\mathrm{H}$-squaring operations. Mem. Fac. Sci. Kyusyu Univ. Ser. A, 1956.

4. W. Browder, Homology operations and loop spaces. Illinois J. Math., 1960.

5. A Dold, Uber die Steenrodschen Kohomologieoperationen. Ann. of Math., 1961.

6. E. Dyer and R. K. Lashof, Homology of iterated loop spaces. Amer. J. Math., 1962.

7. D. B. A. Epstein, Steenrod operations in homological algebra. Invent. Math. I, 1966.

8. G. Hirsch, Quelques proprietes des produits de Steenrod. C.R. Acad. Sci. Paris, 1955.

9. N. Jacobson, Lie Algebras. Interscience Publishers. 1962.

10. S. Kochman, Ph. D. Thesis. University of Chicago, 1970.

11. D. Kraines, Massey higher products. Trans. Amer. Math. Soc. 1966.

12. T. Kudo, A transgression theorem. Mem. Fac. Sci. Kyusyu Univ. Ser. A, 1956.

13. A. Liulevicius, The factorization of cyclic reduced powers by secondary cohomology operations. Mem. Amer. Math. Soc., 1962.

14. S. MacLane, Homology. Academic Press, 1963. 
15. J.P. May, Simplicial objects in algebraic topology. D. Van Nostrand npany, 1967.

16. , The structure and applications of the Eilenberg-Moore

ctral sequences (to appear).

17. - Homology operations in iterated loop spaces (to appear).

18. , The cohomology of the Steenrod algebra (to appear).

19. J. Milnor and J.C. Moore, On the structure of Hopf algebras. Ann. of h. , 1965.

20. J.C. Moore, Constructions sur les complexes d'anneaux. Seminaire ri Cartan, 1954/55.

21. G. Nishida, Cohomology operations in iterated loop spaces. Proc. in Acad., 1968.

22. S. Priddy, Primary cohomology operations for simplicial Lie algebras. ppear in IIl. J. Math.

23. J.P. Serre, Cohomologie modulo 2 des complexes d'Eilenberg-MacLane. ment. Math. Helv., 1953.

24. E. Spanier, Algebraic Topology. McGraw Hill Book Company, 1966. :5. N.E. Steenrod, Products of cocycles and extensions of mappings. of Math., 1947.

6. , Reduced powers of cohomology classes. Ann. of Math.,

7. - Homology groups of symmetric groups and reduced power :ions. Proc. Nat. Acad. Sci., U.S. A., 1953. 3. , Cyclic reduced powers of cohomology classes. Proc. lcad. Sci., U.S.A., 1953. 3. - Cohomology operations derived from the symmetric group. rent. Math. Helv., 1957. 
30. N. E. Steenrod, Cohomology operations. Princeton University Press, 1962. 